\title{
OPTIMAL MORTGAGE PREPAYMENT UNDER THE COX-INGERSOLL-ROSS MODEL
}

by

\section{Christopher Scott Jones}

B.S., Youngstown State University, 2003

M.A., University of Pittsburgh, 2007

\author{
Submitted to the Graduate Faculty of \\ the Department of Mathematics in partial fulfillment \\ of the requirements for the degree of \\ Doctor of Philosophy
}

University of Pittsburgh

2012 


\title{
UNIVERSITY OF PITTSBURGH DEPARTMENT OF MATHEMATICS
}

This dissertation was presented

by

\author{
Christopher Scott Jones
}

It was defended on

August 24, 2012

and approved by

Professor Xinfu Chen, Department of Mathematics

Professor John Chadam, Department of Mathematics

Professor Huiqiang Jiang, Department of Mathematics

Professor Jean-François Richard, Department of Economics

Dissertation Director: Professor Xinfu Chen, Department of Mathematics 
Copyright (C) by Christopher Scott Jones

2012 


\title{
OPTIMAL MORTGAGE PREPAYMENT UNDER THE COX-INGERSOLL-ROSS MODEL
}

\author{
Christopher Scott Jones, PhD
}

University of Pittsburgh, 2012

We study a parabolic variational inequality and associated free boundary problem (FBP) with financial applications. We consider a mortgage contract having the early termination option via prepayment under the Cox-Ingersoll-Ross (CIR) model for the short rate. The problem is of significant interest from both mathematical and economic points of view. The main difficulty is that the Black-Scholes-type partial differential equation (PDE) associated with the problem is not uniformly parabolic and therefore standard approaches have to be modified to treat the degeneracy of the PDE.

Our main results are the existence and uniqueness of a solution to the variational inequality and the associated FBP; the free boundary represents optimal prepayment rate for the mortgage contract at each time prior to expiry. We establish regularity of the free boundary; several other properties of the free boundary are studied as well as the infinite horizon problem.

From the financial point of view, the infinite horizon problem is interpreted as the mortgage prepayment problem when there is a long time to expiration. In solving the infinite horizon problem we obtain a critical rate, such that for mortgage rates below this critical rate, prepayment is never an optimal decision for a long times to expiration.

With the existence of a unique solution to the FBP established, we conclude by performing a calibration of the CIR model to constant maturity Treasury yields and investigate numerical aspects of the variational inequality. 


\section{TABLE OF CONTENTS}

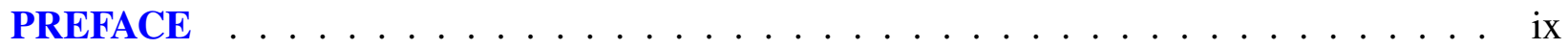

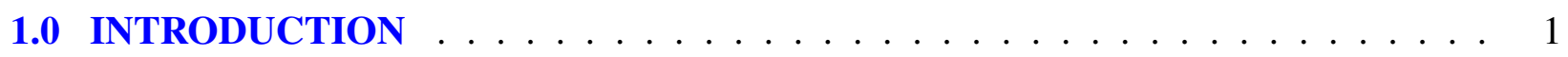

2.0 THE DERIVATION AND FORMULATION OF THE PROBLEM $\ldots \ldots \ldots \ldots$

2.1 Formulation and Derivation $\ldots \ldots \ldots \ldots \ldots$

2.2 The Affine Term Structure Model (ATSM) $\ldots \ldots \ldots \ldots$

2.3 The Hedging Strategy $\ldots \ldots \ldots \ldots$

3.0 THE INFINITE HORIZON PROBLEM . . . . . . . . . . . . . . . . . . . . . . . 11

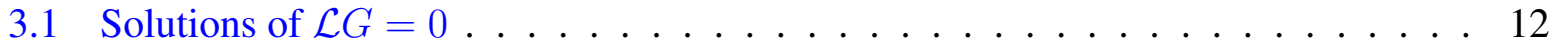

3.2 The Weak Formulation . . . . . . . . . . . . . . . . . 12

3.3 Basic Properties of the Weak Solution . . . . . . . . . . . . . . . . 13

3.4 The Solution for $R_{*} \ldots \ldots \ldots \ldots \ldots \ldots$

3.5 The Solution of the Infinite Horizon Problem . . . . . . . . . . . . . . 18

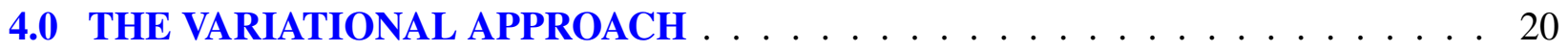

4.1 The Main Result . . . . . . . . . . . . . . . . . . . . . . . . . 20

4.2 Uniqueness . . . . . . . . . . . . . . . . . . 21

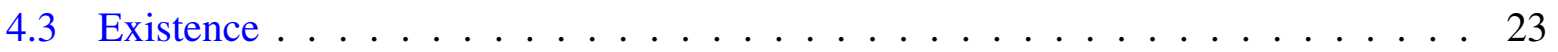

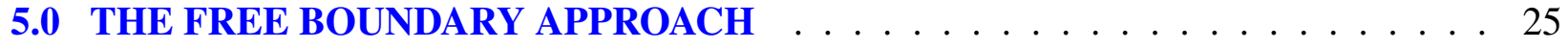

5.1 The Free Boundary Problem . . . . . . . . . . . . . . . 25

5.2 Regularized Problem . . . . . . . . . . . . . . . . . . . . 27

5.3 Small Time Behavior $\ldots \ldots \ldots \ldots \ldots \ldots \ldots \ldots$

5.4 The Limit of the Approximation Problem . . . . . . . . . . . . . . . . . . . 32

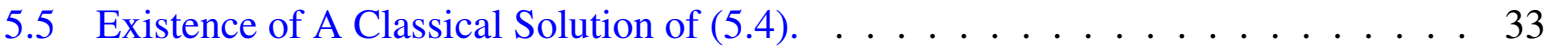


5.6 The Non-uniqueness Issue $\ldots \ldots \ldots$

5.7 Equivalency of the Free Boundary Problem (5.4) and the Variational Inequality (2.1) 36

5.8 The function $W_{\tau} \ldots \ldots \ldots \ldots \ldots \ldots \ldots \ldots$

6.0 CALIBRATING THE COX-INGERSOLL-ROSS MODEL . . . . . . . . . . . 39

6.1 Statistical Properties of the CIR Model . . . . . . . . . . . . . . . . . . . . . . . 39

$6.2 \quad$ U.S. Treasury Data . . . . . . . . . . . . . . . . . . . . . . . . . 41

6.3 Time Series Analysis . . . . . . . . . . . . . . . . . . . . . . . . 43

6.4 Cross-Section Analysis . . . . . . . . . . . . . . . . . . . 51

6.5 Remarks on Estimation Methods . . . . . . . . . . . . . . . . . . . . . 53

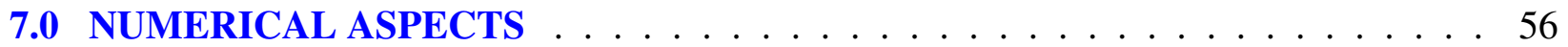

7.1 The Free Boundary Near Expiration . . . . . . . . . . . . . . . . . 56

7.2 Transformations to Canonical Forms _. . . . . . . . . . . . . . . . 59

7.3 Discretization and Implementation $\ldots \ldots \ldots$

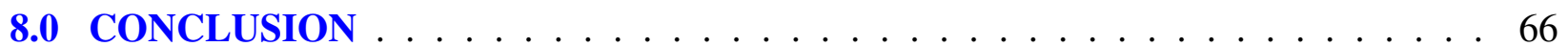

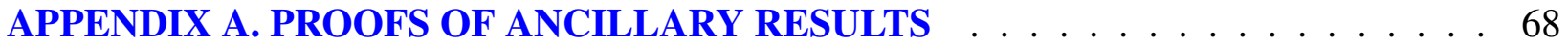

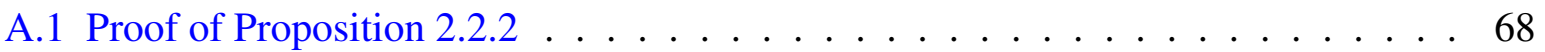

A.2 The Fundamental Solution and Derivation of the Transition Density Function . . 69

A.3 Derivation Green's Formula and Bond Prices . . . . . . . . . . . . . . . . . 71

A.4 Proof of Lemma $2.1 .1 \ldots \ldots$

APPENDIX B. EXTENSIONS OF THE PROBLEM . . . . . . . . . . . . . . . . . . . . 74

B.1 General Affine Term Structure Models . . . . . . . . . . . . . . . . . . . 74

B.2 An Integral Equation Approach . . . . . . . . . . . . . . . . . . . 75

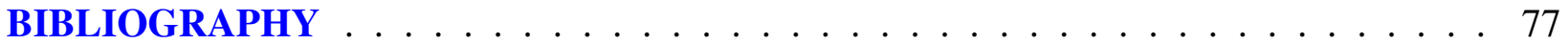




\section{LIST OF TABLES}

1 U.S. Treasury yields from January 1, 2009 to December 31, 2011 . . . . . . . . . 42

2 Maximum likelihood estimates for the entire data set. . . . . . . . . . . . . . 49

3 Maximum likelihood estimates for the simulated CIR processes. . . . . . . . . . . 50

4 Parameter estimates using the data grouping approach. . . . . . . . . . . 50

5 Results for daily cross-section analysis. . . . . . . . . . . . . . . . . . 52

6 Parameter estimates for the estimated short rate. . . . . . . . . . . 53

7 Parameter estimates using the composite method. . . . . . . . . . . . . . . . . 54 


\section{LIST OF FIGURES}

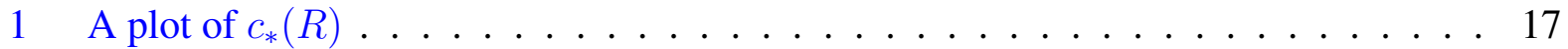

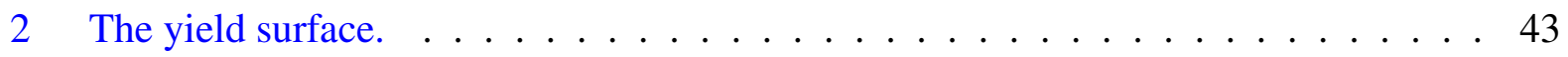

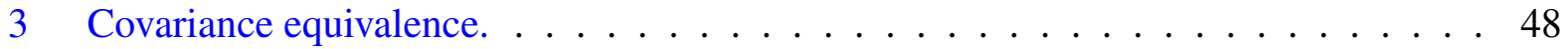

4 A comparison of observed and simulated interest rates. . . . . . . . . . . . . . 49

5 Comparison of the cross-section estimated short rate. . . . . . . . . . . . . . 54

6 The asymptotic free boundary for $\tau \in[0,0.05] \ldots \ldots \ldots \ldots$

7 Approximation of the Optimal Termination Boundary . . . . . . . . . . . . 65 


\section{PREFACE}

Throughout the course of my doctoral work, I have received guidance and encouragement from my advisor, Professor Xinfu Chen. I have learned a great deal from his expertise and energetic passion for mathematics. Along with his enthusiasm, Xinfu's good sense of humor has made this endeavor very enjoyable - I am pleased to thank him for his time and mentorship, this thesis would not have been possible without him.

I am also indebted to my dissertation committee - Professors Jean-Franogis Richard, John Chadam and Huiqiang Jiang for comments, insights and suggestions which have improved this work. Also, I would like to thank Professor Bill Troy for an enjoyable and beneficial course on special functions and asymptotic analysis, the subject matter of which has been useful time again throughout my doctoral work.

I am also grateful to the Department of Mathematics and the University of Pittsburgh for their generous support throughout my graduate career and for the nourishing academic environment that they have provided.

Finally, it is my pleasure to thank my parents, Martha and Robert. I am forever grateful for their constant support and encouragement throughout my entire education. They are truly wonderful people and great parents. This thesis is dedicated to them. 


\subsection{INTRODUCTION}

Since the innovative work of Black, Scholes and Merton, continuous-time stochastic models have been repeatedly employed to successfully reveal theoretical insights into the pricing and risks of financial instruments. Following an initial focus on equity options and other derivatives, this body of work has been expanded to study the prepayment risk associated with fixed rate mortgages.

A (fixed rate) mortgage loan is a contract which allows the borrower (mortgagor) to obtain funds from a financial institution (mortgagee) with a certain repayment schedule. We consider a mortgage contract with a regular repayment schedule of continuous pay-rate $m$ per unit time up to time $T$, the maturity of the mortgage contract. The amount of funds that can be borrowed depends on a fixed interest rate $c$ and the term $T$ of the mortgage. From time $t$ to $t+d t \leqslant T$, the balance $B(t)$ of the mortgage increases by the amount of mortgage interest $c B(t) d t$, less the payment $m d t$, thus

$$
d B=(c B-m) d t
$$

Since, the mortgage loan is amortized, the remaining balance is zero, i.e., we have the terminal condition $B(T)=0$ so we can solve the differential equation and obtain that the amount that can be borrowed at $t<T$ is

$$
B(t)=m\left(1-e^{-c(T-t)}\right) c^{-1}
$$

We consider a contract for which the borrower has the option to pay the outstanding balance $B(s)$ at any time $s \in(t, T]$, thus terminating the contract.

In practice, the borrower's decision to terminate, or prepay, the mortgage contract early (by paying the outstanding balance) depends on many factors, e.g., sale of the collateral for example. 
In theory, this decision depends on the information given by the yield curve. Roughly speaking, if the interest rates (of various specific terms) are sufficiently lower than the mortgage rate $c$, the borrower may find an alternative "cheaper" loan to replace the existing mortgage, i.e., refinance the loan. On the other-hand, if the interest rates are higher relative to the interest rate of the mortgage, it is favorable to continue the mortgage contract by continuing with the scheduled pay-rate $m$, i.e., making an investment instead of terminating the mortgage. Thus, falling interest rates increase the incentive for borrowers to prepay their mortgages, making interest rate risk a primary market risk factor associated with the mortgage market.

For the mortgagee, in order to evaluate its capital asset (especially in reconstruction or in an unfortunate liquidation process) there is a need to evaluate the true value of the mortgage contract, taking into account the prepayment risk.

To evaluate a mortgage contract having the option of early termination, we assume that yields of bonds are affine functions [19] of the short-term interest rate, $r_{t}$. We assume the short-rate is described by the Cox-Ingersoll-Ross (CIR) model [15]:

$$
d r_{t}=\kappa\left(\theta-r_{t}\right) d t+\sigma \sqrt{r_{t}} d \hat{W}_{t}
$$

where, under risk-neutral measure, $d \hat{W}_{t}$ is a standard Brownian motion. The quantities $\kappa, \theta$ and $\sigma$, which represent the mean-reversion speed, long-term mean and volatility of the short-rate, are assumed to be constant.

As shown in the next chapter, the value of the mortgage contract at time $t \leqslant T$ depends only on $r_{t}$; more precisely, the value is $V\left(r_{t}, t\right)$ where $V(\cdot, \cdot)$, a function defined on $[0, \infty) \times(-\infty, T]$, is the solution of the variational inequality

$$
\left\{\begin{array}{l}
\min \left\{\frac{\partial}{\partial t} V+\mathcal{L} V+m, B-V\right\}=0 \quad \text { on }(0, \infty) \times(-\infty, T) \\
V(\cdot, T)=0
\end{array}\right.
$$

where the operator $\mathcal{L}$ is defined by

$$
\mathcal{L} \phi:=\frac{\sigma^{2} r}{2} \frac{\partial^{2} \phi}{\partial r^{2}}+\kappa(\theta-r) \frac{\partial \phi}{\partial r}-r \phi
$$


Early work on the pricing of default-free fixed rate mortgages in a continuous-time framework by Hendershott and Buser can be found in [6]. Kau and Keenan's work on pricing mortgages from the option-theoretic point of view is overviewed in [32].

Analysis of the optimal termination boundary (for the borrower) and evaluation of the mortgage contract (for the lender) has been performed in the case where the short-rate is described by the Vasicek model [38]. In particular, Jiang, Bian and Yi [30] established that in the case of the Vasicek model, the problem is well-posed and the optimal termination boundary $r=R(t)$ is smooth in $(-\infty, T)$ and $R(t)=c-[\alpha+o(1)] \sqrt{T-t}$ as $t \nearrow T$ where $\alpha$ is a positive constant. Their work was extended by Xie, Chen and Chadam [39], where an asymptotic expansion for $R(t)$ as $t \rightarrow-\infty$ and analytic approximation formulas for $R(t)$ uniform in $t \in(-\infty, T]$ were derived, and accurate numerical methods were demonstrated.

A well-known aspect of the Vasicek model of the short-rate is the potential for the short rate to become negative. Under the CIR model, interest rates are always non-negative. Moreover, the CIR model has additional attractive features such as increased volatility with higher levels of interest rates together with a mean-reversion property.

Upon inspection of (1.1), we find that a principal difficulty is that the operator $\mathcal{L}$ for the mortgage prepayment problem under CIR process is not uniformly parabolic. Thus, standard approaches to variational inequalities need to be modified before we can establish the existence and uniqueness of a solution to (1.1). Jiang, Bian and Yi addressed the problem under the assumption of the Vasicek model of the short-rate [30].

In this thesis, we carry the work in [30] to the CIR model. We shall show that (1.1) admits a unique (Lipschitz continuous) solution. For the infinite horizon problem, we show that there exists $c^{*}>0$ such that when $c>c^{*}$, then there exists $R^{*}>0$ such that $V_{*}<1$ in $\left(R^{*}, \infty\right)$ and $V_{*}=1$ in $\left[0, R^{*}\right]$. In the case $c \in\left(0, c^{*}\right]$, we have $V_{*}=c V_{* *} / c^{*}$ where $V_{* *}$ is the solution of the infinite horizon problem with $c=c^{*}$, having the property that $V_{* *}<1$ in $(0, \infty), V_{* *}(0)=1$ and $V_{* *}^{\prime}(0)=-\kappa \theta / c^{*}$. Consequently, when $c \geqslant c^{*}$, we have $R(t)>R^{*}$ for all $t<T$ and $R(t) \rightarrow R^{*}$ as $t \rightarrow-\infty$. When $c \in\left(0, c^{*}\right)$, there exists $t^{*}$ such that $R(t)>0$ when $t \in\left(t^{*}, T\right)$ and $R(t)=0$ when $t<t^{*}$. This unique result implies that when $t<t^{*}$, there is no need to consider early termination of the mortgage contract, no matter how small $r_{t}$ is!

Broadly speaking from the economic perspective, in an option theoretic model such as the 
one described in this thesis, borrowers are considered to own an option - the option to prepay their mortgage - which they exercise optimally, as described above. Though we do acknowledge that optimal exercise in the continuous-time sense of the CIR model does not necessarily result in realistic modeling of the prepayment incentive, the choice between borrowers exercising the prepayment option and continuing to hold their prepayment option is made relative to a valuation of the mortgage contract.

From the mathematical perspective, the aforementioned problem does not appear to have been sufficiently resolved. To this end, we will begin with a formulation of the problem using a BlackScholes type hedging argument, which appears to be a new contribution to the literature. 


\subsection{THE DERIVATION AND FORMULATION OF THE PROBLEM}

A key feature of this chapter is our mathematical formulation of the problem and our derivation of a Black-Scholes type hedging argument for the prepayment problem. This approach does not appear in previous literature on the prepayment problem.

\subsection{FORMULATION AND DERIVATION}

We first note that the solution is linear in $m$, so, in the sequel, we assume without loss of generality that $m=1$. Then

$$
B(t)=M(T-t), \quad M(\tau):=\left(1-e^{-c \tau}\right) c^{-1}
$$

We introduce the following quantities together with their financial interpretations:

$$
\begin{aligned}
\text { time-to-expiry: } & \tau:=T-t, \\
\text { termination-balance: } & M(\tau):=\left[1-e^{-c \tau}\right] c^{-1}, \\
\text { pre-termination-loss: } & W(r, \tau)=M(\tau)-V(r, T-\tau), \\
\text { discounted-interest-pay-rate: } & P(r, \tau):=(c-r) M(\tau), \\
\text { Black-Scholes' operator: } & \mathcal{L}:=\frac{1}{2} \sigma^{2} r \partial_{r r}+\kappa(r-\theta) \partial_{r}-r .
\end{aligned}
$$

Then (1.1) can be written as

$$
\min \left\{W_{\tau}-\mathcal{L} W+P, \quad W\right\}=0 \text { on }(0, \infty)^{2}, \quad W(\cdot, 0)=0
$$


For easy reference, we list the constants to be used in this paper:

$$
b=\frac{2 \kappa \theta}{\sigma^{2}}, \quad \nu=\frac{2 \kappa}{\sigma^{2}}, \quad \gamma=\sqrt{\kappa^{2}+2 \sigma^{2}}, \quad \lambda=\frac{\gamma+\kappa}{\sigma^{2}}, \quad \mu=\frac{\gamma-\kappa}{\sigma^{2}}, \quad a=\frac{\mu b}{\lambda+\mu} .
$$

In the following, by classical solution we mean every derivative appearing in the equation exists in the classical sense.

\subsection{THE AFFINE TERM STRUCTURE MODEL (ATSM)}

In defining an affine term structure model, we begin with our definition of a zero coupon bond.

A $T$-bond is a guaranteed unit payment at time $T$. The One Factor ATSM [19] assumes that the yields of bonds are affine functions of a stochastic factor, that is, there are smooth functions $A(\cdot)(\not \equiv 0)$ and $B(\cdot)$ and a non-constant stochastic process $\left\{F_{t}\right\}$ such that the price, $Z_{t}^{T}$, of a $T$-bond at time $t$ obeys

$$
\left\{\begin{array}{l}
Z_{t}^{T}=e^{A(T-t) F_{t}+B(T-t)} \quad \forall t>0, T \geqslant t, \\
d F_{t}=\mu_{t} d t+\sigma_{t} d W_{t}
\end{array}\right.
$$

where $\left\{W_{t}\right\}$ is the Standard Brownian motion and $\left\{\mu_{t}, \sigma_{t}\right\}$ are certain stochastic processes.

As shown by Duffie and Kan [19], an ATSM requires substantial restrictions on $A, B$, and $\sigma_{t}$. First of all, as $Z_{t}^{t}=1$, we have $A(0) F_{t}+B(0)=0$, so $A(0)=0, B(0)=0$. Next, for any fixed $T$, as a stochastic process of $t$, we have, writing $\tau=T-t$,

$$
\begin{gathered}
d Z_{t}^{T}=Z_{t}^{T}\left(R_{t}^{T} d t+\sigma_{t}^{T} d W_{t}\right) \\
\sigma_{t}^{T}:=\sigma_{t} A(\tau), \quad R_{t}^{T}:=A(\tau) \mu_{t}+A^{2}(\tau) \sigma_{t}^{2} / 2-A^{\prime}(\tau) F_{t}-B^{\prime}(\tau)
\end{gathered}
$$

Heath, Jarrow and Morton [26] proved the following for single factor ATSMs:

Proposition 2.2.1. [The Fundamental Theorem of Asset Pricing, [26]] For a single factor ATSM (2.3), there exist processes $\left\{r_{t}, \lambda_{t}\right\}$ such that $R_{t}^{T}-r_{t}=\lambda_{t} \sigma_{t}^{T}$. That is,

$$
B^{\prime}(\tau)+A^{\prime}(\tau) F_{t}+r_{t}+\left(\lambda_{t} \sigma_{t}-\mu_{t}\right) A(\tau)-A^{2}(\tau) \sigma_{t}^{2} / 2=0
$$


Using the Heath-Jarrow-Morton result, we prove the following relationship between short rate models and the single factor ATSMs.

Proposition 2.2.2. In a single factor ATSM model, there are constants $\sigma_{0}, \sigma_{1}, k_{0}, k_{1}$ and a stochastic process $\left\{\lambda_{t}\right\}$ such that

$$
d r_{t}=\hat{\mu}_{t} d t+\sigma_{t} d \hat{W}_{t}
$$

where the $\hat{\mu}_{t}, \hat{\sigma}_{t}^{2}$ and $\hat{W}_{t}$ are given by

$$
\hat{\mu}_{t}=k_{0}+k_{1} r_{t}, \quad \sigma_{t}^{2}=\sigma_{0}+\sigma_{1} r_{t}, \quad \hat{W}_{t}=W_{t}+\int_{0}^{t} \lambda_{s} d s .
$$

The T-bond price is then given by

$$
Z_{t}^{T}=e^{A(T-t) r_{t}+B(T-t)}, \quad d Z_{t}^{T}=Z_{t}^{T}\left[r_{t} d t+A(T-\tau) \sigma_{t} d \hat{W}_{t}\right]
$$

where the functions $A$ and $B$ satisfy the Riccati-type equations

$$
A^{\prime}=\frac{\sigma_{1}^{2}}{2} A^{2}-k_{1} A-1, A(0)=0, \quad B(\tau)=\int_{0}^{\tau}\left[\frac{\sigma_{0}^{2}}{2} A^{2}-k_{0} A\right] .
$$

The proof is left to the appendix.

For the CIR model [15], we have

$$
k_{0}=\kappa \theta, \quad k_{1}=-\kappa, \quad \sigma_{0}=0, \quad \sigma_{1}=\sigma .
$$

Note that the yield of the $(t+d t)$-bond at time $t$ is $-\frac{1}{d t} \log Z_{t}^{t+d t}=-A^{\prime}(0) r_{t}+B^{\prime}(0)=r_{t}$.

Definition 2.2.1. We call the portfolio of investing all money in $(t+d t)$-bond at every time $t$ the money market account.

Remark 2.2.1. For asset pricing in mathematical finance, the measure under which $\hat{W}_{t}$ is a martingale is called the risk-neutral measure, for which the process $\left\{\lambda_{t}\right\}$ (the market price of risk) plays no role. Nevertheless, in statistical estimation of the parameters, one must use the natural measure under under which $\left\{W_{t}\right\}$ is a martingale. Being the solution of the stochastic differential equation

$$
d r_{t}=\mu_{t} d t+\sqrt{\sigma_{0}+\sigma_{1} r_{t}} d W_{t}
$$

with $\mu_{t}=k_{0}+k_{1} r_{t}+\lambda_{t} \sigma_{t}$ which in theory can be arbitrary, the solution $\left\{r_{t}\right\}$ can take a wide variety of forms. 


\subsection{THE HEDGING STRATEGY}

In this section we provide a hedging strategy showing that the no-arbitrage price of the mortgage at time $t$ is $V\left(r_{t}, t\right)$, for each $t \leqslant T$, where $V$ is the solution of (1.1). Developing a hedging strategy is essential in our analysis as it shows that the no-arbitrage price of the mortgage at time $t$ is indeed $V\left(r_{t}, t\right)$. Constructing a hedging strategy appears to be a new addition to the literature on optimal mortgage prepayment.

Let $V$ be the solution of (1.1) satisfying the following two conditions (so that we may apply Ito's Lemma):

Assumption 1 The function $\partial_{r} V$ is continuous on $[0, \infty) \times[0, T], \mathbb{R}$; and the functions $\partial_{t} V$ and $r \partial_{r r} V$ are bounded.

Assumption 2 There exists $R(\cdot)$ such that $V(r, t)<M(T-t)$ if $r>R(t)$ and $V(r, t)=$ $M(T-t)$ if $r \in[0, R(t))$.

We show that at $t=0$, the mortgage has a no-arbitrage price, $P(0)$, that equals $V\left(r_{0}, 0\right)$.

(i) Suppose $P(0)<V\left(r_{0}, 0\right)$. From the point of view of the mortgagee, she needs to invest in a hedging portfolio that starts from $-P(0)$ and uses the cash flow from the mortgagor to escrow to a non-negative final value at a termination time, say, $S \in(0, T]$, chosen by the mortgagor. Consider the following trading strategy: picking $\hat{T}>T$, as long as the mortgage is not terminated, invest $\alpha_{t}:=-\partial_{r} V\left(r_{t}, t\right) / A(\hat{T}-t)$ amount of funds on the $\hat{T}$-bond with the rest in the money market account. Denote by $P_{t}$ the value of the portfolio at time $t \in[0, S]$. Then

$$
\begin{aligned}
P_{0} & =-P(0)<0, \\
d P_{t} & =m d t+\left\{\alpha_{t} / Z_{t}^{\hat{T}}\right\} d Z_{t}^{\hat{T}}+\left(P_{t}-\alpha_{t}\right) r_{t} d t \\
& =\left\{m+P_{t} r_{t}\right\} d t+\alpha_{t} A(T-t) \sigma_{t} d \hat{W}_{t} \quad \forall t \in(0, s), \\
P_{S} & =P_{S-}+M(T-S) .
\end{aligned}
$$

We now relate $P_{t}$ with $V\left(r_{t}, t\right)$. By Itô's Lemma,

$$
d V\left(r_{t}, t\right)=\left\{\partial_{t} V+\frac{1}{2} \sigma_{t}^{2} \partial_{r r}^{2} V+\hat{\mu}_{t} \partial_{r} V\right\} d t+V_{r}\left(r_{t}, t\right) \sigma_{t} d \hat{W}_{t}
$$


Denoting $\xi(r, t)=V_{t}+\mathcal{L} V+m$, we find that

$$
d\left(V\left(r_{t}, t\right)+P_{t}\right)=\left(P_{t}+V\left(r_{t}, t\right)\right) r_{t} d t+\xi\left(r_{t}, t\right) d t
$$

Solving this ordinary differential equation we obtain

$$
\begin{aligned}
P_{t} & =-V\left(r_{t}, t\right)+\left[V\left(r_{0}, 0\right)-P(0)\right] e^{\int_{0}^{t} r_{s} d s}+\int_{0}^{t} \xi\left(r_{s}, s\right) e^{\int_{\hat{s}}^{t} r_{\hat{s}} d \hat{s}} d s \quad \forall t \in(0, S), \\
P_{S} & =\left[M(T-S)-V\left(r_{S}, S\right)\right]+\left[V\left(r_{0}, 0\right)-P(0)\right] e^{\int_{0}^{t} r_{s} d s}+\int_{0}^{t} \xi\left(r_{s}, s\right) e^{\int_{\hat{s}}^{t} r_{\hat{s}} d \hat{s}} d s .
\end{aligned}
$$

Hence, if initially the mortgagee paid $P(0)\left(<V\left(r_{0}, 0\right)\right)$ for the right to receive the cash flow from the mortgagor, then using the above hedging strategy, at any termination time $S \in(0, T]$, the mortgagee ends up with a profit $P_{S}>0$.

(ii) Next suppose $P(0)>V\left(r_{0}, 0\right)$. From the mortgagor's point of view, after obtaining the amount of cash $P(0)$, he constructs a portfolio to pay off the mortgage. If $P(0)>M(T-0)$, then the mortgagor can terminate the contract at $t=0$ by paying back $M(T-0)$ to the mortgagee and profiting $P(0)-M(T-0)$. If $P(0) \leqslant M(T-0)$, then $V\left(r_{0}, 0\right)<P(0) \leqslant M(T-0)$, so $r_{0}>R(0)$. We can define the stopping time

$$
S:=\inf \left\{t \leqslant T \mid r_{s}>R(s) \forall s \in[0, t]\right\}
$$

One can check that $M(T-S)=V\left(r_{S}, S\right)$ and $\xi\left(r_{s}, s\right)=0$ for $s \in[0, S]$. Thus, if the mortgagor invests $\alpha_{t}=\partial_{r} V\left(r_{t}, t\right) / A(\hat{T}-t)$ in the $\hat{T}$-bond with the rest in the money market account, up to time $S$ to terminate the mortgage, he will pay-off the mortgage at time $S$ with a profit $[P(0)-$ $\left.V\left(r_{0}, 0\right)\right] e^{\int_{0}^{S} r_{t} d t}$.

Based on (i) and (ii), we conclude that the no-arbitrage price of the mortgage at $t=0$ is $V\left(r_{0}, 0\right)$. Continuing inductively in this fashion, we have that at any time $t<T$, the value of the mortgage contract is $V\left(r_{t}, t\right)$. 
Remark 2.3.1. (i) Even with the no-arbitrage price, if the mortgagor does not terminate the mortgage at the first time when $r_{t}$ drops to $R(t)$, the mortgagee still has a profit of at least $\int_{0}^{S} \xi\left(r_{t}, t\right) e^{\int_{t}^{S} r_{s} d s} d t$. Hence, $S$ in $(2.5)$ is the only optimal mortgage termination time.

(ii) If early termination is not allowed, for each time interval $[s, s+d s)$ one buys $m d s$ amount of $s$-bond at time $t$ to hedge the future payment $m d s$. The present value of the cash flow for all the mortgage payments therefore equals

$$
\tilde{M}\left(r_{t}, t\right)=\int_{t}^{T} Z_{t}^{s} m d s=m \int_{0}^{T-t} e^{A(\tau) r_{t}+B(\tau)} d \tau
$$

where explicit formulas for $Z_{t}^{s}, A$ and $B$ can be found in Corollary A.3.3 in the appendix.

For the remainder of this thesis, all subscripts (except $r_{t}$ ) represent partial derivatives. 


\subsection{THE INFINITE HORIZON PROBLEM}

From the economic point of view, there are two times during the life of a mortgage contract where the optimal prepayment strategy is of significant interest: when the contract is close to expiration and when there is a long time to expiration. In this chapter, we analyze the prepayment option when there is a long time to expiration, i.e., soon after the contract is initiated.

From the mathematical point of view, we can model a long to expiry scenario by working on the scaled function $\mathrm{cV}(r, t) / m$ and sending $T \rightarrow \infty$ in the variational problem (1.1). We obtain a limit $V_{*}$ that is a solution of the infinite horizon problem:

$$
\min \left\{\mathcal{L} V_{*}+c, 1-V_{*}\right\}=0 \quad \text { on }(0, \infty)
$$

In this chapter, we follow the ideas of [39] studying the infinite horizon problem (3.1). We do this with the addition of a new definition of a weak solution and treatment of degeneracy of $\mathcal{L}$ at $r=0$.

For a weak solution $V_{*}$, we show that there exists a non-negative constant $R_{*}$ such that $V_{*}<1$

in $\left(R_{*}, \infty\right)$ and in the case $R_{*}>0$ there holds $V_{*} \equiv 1$ in $\left[0, R_{*}\right]$ and $V_{*}^{\prime}\left(R_{*}\right)=0$. Then we solve the problem by means of variation of constants utilizing the solution to the homogeneous equation that we derive below. Finally, regarding $R_{*}$ as a function of $c$, we show that there exists a positive $c^{*}$ such that $R_{*}(c)$ is a strictly increasing function of $c$ when $c \geqslant c^{*}$, whereas when $c \in\left(0, c^{*}\right.$, $R_{*}(c)=0$, and $V_{*}=\left(c / c^{*}\right) V_{* *}$ where $V_{* *}$ is the solution with $c=c^{*}$.

We shall also derive formulas for $c^{*}, R_{*}(c)$ and $V_{* *}$ and therefore prove the uniqueness of the solution. 


\subsection{SOLUTIONS OF $\mathcal{L} G=0$}

Positive solutions of

$$
\mathcal{L} G:=\frac{\sigma^{2}}{2} \frac{d^{2} G}{d r^{2}}+\kappa(\theta-r) \frac{d G}{d r}-r G=0
$$

on $(0, \infty)$ play an important role in our analysis. As the operator $\mathcal{L}$ is of second order with linear coefficients, the Laplace transform of $G$ satisfies a first order linear equation and thus can be solved. The inverse of the Laplace transform leads to various famous special functions. Needless to say, solutions of $\mathcal{L} G=0$ are in the category of those that have been well-studied.

Lemma 3.1.1. The equation $\mathcal{L} G=0$ on $(0, \infty)$ has solutions $G=G_{1}$ and $G=G_{2}$ with the following properties:

$$
\begin{array}{ccc}
\lim _{r \searrow 0} r^{b} G_{1}^{\prime}(r)=-1, & G_{1}>0>G_{1}^{\prime} \text { in }(0, \infty), & \lim _{r \rightarrow \infty} r^{a} e^{\mu r} G_{1}(r)=C_{1} \\
G_{2}(0)=1, & G_{2}^{\prime}(0)=0, \quad G_{2}^{\prime}>0 \text { in }(0, \infty), & \lim _{r \rightarrow \infty} r^{b-a} e^{-\lambda r} G_{2}(r)=C_{2}
\end{array}
$$

where $C_{1}$ and $C_{2}$ are some positive constants and $a, b, \lambda, \mu$ are constants given in (2.2).

The proof is left to the appendix.

\subsection{THE WEAK FORMULATION}

In this section, we formulate (3.1) in a week sense. The variational inequality (3.1) implies that $V_{*} \in C^{\infty}(\Omega)$ is a classical solution of $\mathcal{L} V_{*}+c=0$ in $\Omega:=\left\{r>0 \mid V_{*}(r)<1\right\}$. The differential inequality $\mathcal{L} V_{*} \geqslant 0$ in $(0, \infty)$ can be written as $\left(A V_{*}+B\right)_{r r} \geqslant 0$ where, using $b, \nu$ defined as in (2.2),

$$
A=r^{b / 2} e^{-\nu r / 2}, \quad B=\int_{1}^{r} \int_{1}^{u}\left(\frac{2\left[c-\rho V_{*}(\rho)\right]}{\sigma^{2} \rho} A(\rho)-A^{\prime \prime}(\rho) V_{*}(\rho)\right) d \rho d u .
$$

This leads naturally to the following weak formulation:

Definition 3.2.1. A weak solution of $(3.1)$ is a function $V_{*} \in C([0, \infty)) \cap L^{\infty}((0, \infty))$ such that 1. $V_{*} \leqslant 1$ on $[0, \infty)$ and $\mathcal{L} V_{*}+c=0$ in $\Omega:=\left\{r \mid r>0, V_{*}(r)<1\right\}$, 
2. for $A$ and $B$ defined in (3.2), $A V_{*}+B$ is convex in $(0, \infty)$, and

3. there holds the no flux boundary condition: $\lim _{r \searrow 0} r^{b-1}\left\{V_{*}(r)-V_{*}(0)\right\}=0$.

Remark 3.2.1. If $V_{*} \in C^{1}$, the no-flux boundary condition is equivalent to $\lim _{r}{ }_{0} r^{b} V_{*}^{\prime}(r)=0$.

The no-flux boundary condition is automatically satisfied when $b \geqslant 1$ since $V_{*}$ is bounded.

When $b \in(0,1)$, some sort of condition such as this natural no-flux boundary condition has to be imposed since otherwise we can construct infinite many solutions of the form $V_{*}=v-\ell G_{1}$ where $v$ is a particular bounded solution of $\mathcal{L} v+c=0$ on $(0, \infty), \ell$ is any large positive constant, and $G_{1}$ is the function given in Lemma 3.1.1.

\subsection{BASIC PROPERTIES OF THE WEAK SOLUTION}

In this subsection we establish the following basic properties of a weak solution using classical techniques from ordinary differential equations.

Proposition 3.3.1. Let $V_{*}$ be a weak solution of (3.1). The following holds:

1. There exists $R_{*} \in[0, c)$ such that $V_{*}^{\prime}<0<1-V_{*}$ and $\mathcal{L} V_{*}+c=0$ in $\left(R_{*}, \infty\right)$;

2. If $R_{*}=0$, then $V_{*}^{\prime}(0)=-c /(\kappa \theta)$;

if $R_{*}>0$, then $V_{*} \equiv 1$ in $\left[0, R_{*}\right], V_{*}^{\prime}\left(R_{*}\right)=0$, and $V_{*}^{\prime \prime}\left(R_{*}+\right)=2\left(R_{*}-c\right) / R_{*} \sigma^{2}$;

3. As $r \rightarrow \infty, V_{*}(r)=c r^{-1}+c \kappa r^{-2}+O\left(r^{-3}\right)$ and $V_{*}^{\prime}(r)=-c r^{-2}+O\left(r^{-3}\right)$.

We begin with three technical Lemmas.

Lemma 3.3.1. Suppose $V_{*}<1$ in $(0, \varepsilon]$ for some $\varepsilon>0$. Then $V_{*} \in C^{\infty}([0, \varepsilon])$ and $V_{*}^{\prime}(0)=-c / \kappa \theta$.

Proof. The general solution of $\mathcal{L} V+c=0$ in $(0, \varepsilon]$ is given by $V=V_{0}+c_{1} G_{1}+c_{2} G_{2}$ where $c_{1}, c_{2}$ are arbitrary constants, $G_{1}$ and $G_{2}$ are given by Lemma 3.1.1, and $V_{0}$ is a special solution that is analytic on $\mathbb{R}$, given by the power series $V_{0}=\sum_{n=1}^{\infty} a_{n} r^{n}$ with coefficients defined by

$$
a_{0}=0, \quad a_{1}=-\frac{c}{\kappa \theta}, \quad a_{n+1}=\frac{2\left(\kappa n a_{n}+a_{n-1}\right)}{\sigma^{2}(n+1)(n+b)} \quad \forall n \geqslant 1 .
$$

Note that $G_{2}$ is analytic in $\mathbb{R}$ satisfying $G_{2}(0)=0=G_{2}^{\prime}(0)$, whereas when $b \geqslant 1, G_{1}$ is unbounded near $r=0$ and when $b \in(0,1), r^{b-1}\left[G_{1}(r)-G_{1}(0)\right] \rightarrow 1 /(b-1)$ as $r \searrow 0$. Hence, by the no 
flux boundary condition, we must have $c_{1}=0$. Consequently, $V$ is analytic on $[0, \varepsilon], V_{*}(0)=c_{2}$, and $V_{*}^{\prime}(0)=-c /(\kappa \theta)$. This completes the proof.

Lemma 3.3.2. Suppose $\hat{r}>0$ and $V_{*}(\hat{r})=1$. Then $V_{*}^{\prime}(\hat{r})=0$.

Proof. Consider the convex function $\Phi=A V_{*}+B$. For small positive $h$, we have

$$
\begin{aligned}
0 & \leqslant \Phi(\hat{r}+h)+\Phi(\hat{r}-h)-2 \Phi(\hat{r}) \\
& =A(\hat{r}+h)\left\{V_{*}(\hat{r}+h)-V_{*}(\hat{r})\right\}+A(\hat{r}-h)\left\{V_{*}(\hat{r}-h)-V_{*}(\hat{r})\right\}+O\left(h^{2}\right) \\
& \leqslant A(\hat{r} \pm h)\left\{V_{*}(\hat{r} \pm h)-V_{*}(\hat{r})\right\}+O\left(h^{2}\right)
\end{aligned}
$$

since both $A$ and $B$ are $C^{2}$ functions and $V_{*}(\hat{r})=1$ is a maximum. Hence,

$$
0 \leqslant h^{-1}\left\{V_{*}(\hat{r})-V_{*}(\hat{r} \pm h)\right\} \leqslant O(h)
$$

Sending $h \searrow 0$ we conclude that $V_{*}^{\prime}(\hat{r})=0$.

Lemma 3.3.3. Suppose $\hat{r}>0$ and $V_{*}(\hat{r})=1$. Then $\hat{r} \leqslant c$ and $V_{*} \equiv 1$ on $[0, \hat{r}]$.

Proof. First we show that $V_{*}=1$ in $[0, \hat{r}]$ by using a contradiction argument. Suppose the assertion is not true. Then $V_{*}$ in $[0, \hat{r}]$ admits a negative global minimum, say at $r_{1} \in(0, \hat{r})$, since $V_{*}$ is continuous in $[0, \hat{r}], V_{*}(\hat{r})=1$, and either $V_{*}(0)=1$ or $V_{*}^{\prime}(0)=-c /(\kappa \theta)<0$ (by Lemma 3.3.1). Then $V_{*}^{\prime}\left(r_{1}\right)=0 \leqslant V_{*}^{\prime \prime}\left(r_{1}\right)$. Note that if $V_{*}^{\prime \prime}\left(r_{1}\right)=0$, then differentiating $\mathcal{L} V_{*}+c=0$ gives $\frac{\sigma^{2}}{2} r_{1} V_{*}^{\prime \prime \prime}\left(r_{1}\right)=V_{*}\left(r_{1}\right)>0$. Hence $V_{*}^{\prime}>0$ in $\left(r_{1}, r_{1}+\varepsilon\right)$ for some $\varepsilon>0$. Define

$$
r_{2}=\sup \left\{r \in\left(r_{1}, \hat{r}\right) \mid V_{*}^{\prime}>0 \text { in }\left(r_{1}, r\right)\right\}
$$

Then, for all $r \in\left(r_{1}, r_{2}\right], V_{*}(r)>V_{*}\left(r_{1}\right)$ and $r V_{*}(r)-c>r_{1} V_{*}\left(r_{1}\right)-c=\frac{\sigma^{2}}{2} r_{1} V_{*}^{\prime \prime}\left(r_{1}\right) \geqslant 0$. In self-adjoint form $\mathcal{L} V_{*}+c=0$ can be written as $\left(r^{b} e^{-\nu r} V_{*}^{\prime}\right)^{\prime}=2 \sigma^{-2}\left(r V_{*}-c\right) r^{b-1} e^{-\nu r}$. Note that the right-hand side is positive on $\left(r_{1}, r_{2}-\right]$ so $V_{*}^{\prime}>0$ in $\left(r_{1}, r_{2}-\right]$. By the maximality of $r_{2}$, we must have $V_{*}\left(r_{2}\right)=1$. However, this contradicts Lemma 3.3.2 which shows that $V_{*}^{\prime}\left(r_{2}\right)=0$. Hence, we must have $V_{*} \equiv 1$ in $[0, \hat{r}]$. Consequently, the convexity of the function $A V_{*}+B$ which is differentiable in $[0, \hat{r}-]$ gives $c-\hat{r} \geqslant 0$. This completes the proof of Lemma 3.3.3. 
Proof of Proposition 3.3.1. Define

$$
R_{*}:= \begin{cases}0 & \text { if } V_{*}<1 \text { in }(0, \infty), \\ \sup \left\{\hat{r}>0 \mid V_{*}(\hat{r})=1\right\} & \text { otherwise. }\end{cases}
$$

If $R_{*}=0$, then by Lemma 3.3.1, $V_{*}$ is analytic in $[0, \infty)$ and $V_{* r}(0)=-c / \kappa \theta<0$.

If $R_{*}>0$ then by Lemma 3.3.3, $R_{*} \in(0, c]$ and that $V_{*} \equiv 1$ in $\left[0, R_{*}\right]$, and by the definition of $R_{*}, V_{*}<1$ in $\left(R_{*}, \infty\right)$. In addition, by Lemma 3.3.2 $V_{*}^{\prime}\left(R_{*}\right)=0$. Thus $V_{*}$ is the solution of $\mathcal{L} V_{*}+c=0$ on $\left(R_{*}, \infty\right)$ subject to the boundary condition at $V_{*}\left(R_{*}\right)=1, V_{*}^{\prime}\left(R_{*}\right)=0$, so it is analytic in $\left[R_{*}, \infty\right)$, and $V_{*}^{\prime \prime}\left(R^{*}+\right)=2\left(R_{*}-c\right) / R_{*} \sigma^{2} \geqslant 0$. We cannot have $V_{*}^{\prime \prime}\left(R^{*}\right)=0$ since it would imply $R_{*}=c, V_{*}^{\prime}\left(R^{*}\right)=0, V_{*}^{\prime \prime}\left(R^{*}+\right)=0$ and $V_{*}^{\prime \prime \prime}\left(R^{*}+\right)=2 / \sigma^{2}>0$ which excludes $R^{*}$ from being a local maximum of $V_{*}$. Thus, $R^{*}<c$.

Next we claim that $V_{*}^{\prime}<0$ in $\left(R_{*}, \infty\right)$. In the case $R_{*}=0$ we have $V_{*}^{\prime}(0)<0$ and in the case $R_{*}>0$, we have $V_{*}^{\prime \prime}\left(R_{*}+\right)<0$, so $V_{*}^{\prime}<0$ in $\left(R_{*}, R_{*}+\varepsilon\right)$ for some $\varepsilon>0$. If the claim were not true, then $r_{1}:=\inf \left\{r>R_{*} \mid V_{*}^{\prime}(r)>0\right\}$ is well-defined and $V_{*}^{\prime}\left(r_{1}\right)=0 \leqslant V_{*}^{\prime \prime}\left(r_{1}\right)$. Define $r_{2}=\sup \left\{r>r_{1} \mid V_{*}^{\prime} \geqslant 0\right.$ in $\left.\left[r_{1}, r\right]\right\}$. Following the same proof as that for Lemma 3.3.3, we can conclude that $r^{b} e^{-\nu r} V_{*}^{\prime}$ is an increasing function on $\left(r_{1}, r_{2}\right)$ so $r_{2}=\infty$. But this would imply $V_{*}$ unbounded. Thus, we must have $V_{*}^{\prime}<0$ in $\left(R_{*}, \infty\right)$.

Finally, we establish the asymptotic behavior of $V_{*}(r)$ as $r \rightarrow \infty$. Let $G_{2}$ be the solution of $\mathcal{L} G=0$ given by Lemma 3.1.1, which is positive and grows exponentially fast as $r \rightarrow \infty$. As $0 \leqslant V_{*} \leqslant 1$, for sufficiently large constant $R$ we can compare $V_{*}$ with $V^{ \pm}=c r^{-1}+c \kappa r^{-2} \pm$ $\left[R^{3} r^{-3}+\varepsilon G_{2}\right]$ for the linear elliptic equation $\mathcal{L} V_{*}+c=0$ on $[R, \infty)$ to conclude that $V_{-}<V_{*}<V_{+}$ so $\left|V_{*}-c r^{-1}-c \kappa r^{-2}\right| \leqslant R^{3} r^{-3}+\varepsilon G_{2}(r)$ for every $r \in[R, \infty)$ and every $\varepsilon>0$. Sending $\varepsilon \searrow 0$ we find that $\left|V_{*}-c r^{-1}-c \kappa r^{-2}\right| \leqslant R r^{-3}$ for any $r \in[R, \infty)$. The asymptotic behavior for $V_{r}^{*}(r)=-c r^{-2}+O\left(r^{-3}\right)$ can be obtained by a similar comparison for the elliptic equation, for $V_{*}^{\prime}$, obtained by differentiating $\mathcal{L} V_{*}+c=0$ and replacing $V_{*}$ by $-c r^{-1}+c \kappa r^{-2}+O\left(r^{-3}\right)$. This completes the proof. 


\subsection{THE SOLUTION FOR $R_{*}$}

In this section we derive a formula for the free boundary $R_{*}$ and $V_{*}\left(R_{*}\right)$ of the infinite horizon problem.

Let $G_{1}$ be the function given in Lemma 3.1.1, the positive solution of $\mathcal{L} G=0$ that decays exponentially fast as $r \rightarrow \infty$. Consider the weighted Wronskian $W(r)=\left\{V_{*}^{\prime} G_{1}-G_{1}^{\prime} V_{*}\right\} r^{b} e^{-\nu r}$. Using the differential equations for $\mathcal{L} V_{*}=-c$ we derive that $\sigma^{2} W^{\prime}=-2 c r^{b-1} e^{-\nu r} G_{1}(r)$ so

$$
W(r)=\frac{2 c}{\sigma^{2}} \int_{r}^{\infty} \rho^{b-1} e^{-\nu \rho} G_{1}(\rho) d \rho \quad \forall r>R_{*} .
$$

Since $V_{*}$ is analytic in $\left[R^{*}, \infty\right),\left(G_{1}^{\prime} r^{b} e^{\nu r}\right)^{\prime}=G_{1} r^{b} e^{\nu r}$, and $\lim _{r \searrow 0} r^{b} G_{1}(r)=0$, we see that

$$
\begin{aligned}
\lim _{r \searrow R_{*}} W(r) & =-V_{*}\left(R_{*}\right) \lim _{r \searrow R_{*}} G_{1}^{\prime}(r) r^{b} e^{-\nu r} \\
& =V_{*}\left(R_{*}\right) \lim _{r \searrow R_{*}} \int_{r}^{\infty}\left(G_{1}^{\prime} \rho^{b} e^{-\nu \rho}\right)^{\prime} d \rho=\frac{2 V_{*}\left(R_{*}\right)}{\sigma^{2}} \int_{R_{*}}^{\infty} \rho^{b} e^{-\nu \rho} G_{1} d \rho .
\end{aligned}
$$

Therefore, sending $r \searrow R_{*}$ in (3.3) we find that $\left(R_{*}, V_{*}\left(R_{*}\right)\right)$ satisfies

$$
\int_{R_{*}}^{\infty}\left\{V_{*}\left(R_{*}\right) \rho-c\right\} \rho^{b-1} e^{-\nu \rho} G_{1}(\rho) d \rho=0, \quad \min \left\{R_{*}, 1-V_{*}\left(R_{*}\right)\right\}=0
$$

Lemma 3.4.1. Problem (3.4), for $\left(R_{*}, V\left(R_{*}\right)\right)$, admits a unique solution. In addition, setting

$$
c^{*}=\frac{\int_{0}^{\infty} r^{b} e^{-\nu r} G_{1}(r) d r}{\int_{0}^{\infty} r^{b-1} e^{-\nu r} G_{1}(r) d r}
$$

and regarding the solution as a function of $c>0$, we have the following:

(1) When $c \in\left(0, c^{*}\right]$, we have $R_{*}=0$ and $V_{*}(0)=c / c^{*}$.

(2) When $c>c^{*}$, we have $R_{*}>0$. Also, as function of $c$, the inverse of $R_{*}=R_{*}(c)$ is given by

$$
c=c\left(R_{*}\right):=\frac{\int_{R_{*}}^{\infty} r^{b} e^{-\nu r} G(r) d r}{\int_{R_{*}}^{\infty} r^{b-1} e^{-\nu r} G(r) d r} \quad \forall R_{*} \geqslant 0
$$


Proof. Define the function

$$
\Psi(c, r) \stackrel{\text { def }}{=} \int_{r}^{\infty}(\rho-c) \rho^{b-1} e^{-\nu \rho} G_{1}(\rho) d \rho \quad \forall c>0, r \geqslant 0
$$

Then $\Psi_{r}(c, r)=(c-r) r^{b-1} e^{-\nu r} G_{1}(r)$ so for each fixed $c>0$, as a function of $r$,

$$
\lim _{r \rightarrow \infty} \Psi(c, r)=0, \quad \Psi_{r}(c, \cdot)<0 \text { in }(c, \infty), \quad \Psi_{r}(c, \cdot)>0 \text { in }(0, c)
$$

Thus, $\Psi(c, \cdot)$ is positive in $[c, \infty)$ and strictly increasing on $[0, c]$. In addition,

$$
\Psi(c, 0)=\int_{0}^{\infty}(\rho-c) \rho^{b-1} e^{-\nu \rho} G_{1}(\rho) d \rho=\left(c^{*}-c\right) \int_{0}^{\infty} \rho^{b-1} e^{-\nu \rho} G_{1}(\rho) d \rho
$$

Thus we conclude the following:

(i) If $0<c \leqslant c^{*}$, then $\Psi(c, \cdot)>0$ in $(0, \infty)$. Consequently, $R_{*}>0$ is impossible. Thus, the only solution of (3.4) is given by $R_{*}=0$ and $V_{*}(0)=c / c^{*}$.

(ii) If $c>c^{*}$, then $\Psi(c, 0)<0$. Consequently, if $\left(R_{*}, V_{*}\left(R_{*}\right)\right)$ is a solution of (3.4), we cannot have $R_{*}=0$; namely, we must have $R_{*}>0$. Hence, $V\left(R_{*}\right)=1$ and $R_{*}$ is the unique root of $\Psi(c, \cdot)=0$ in $(0, c)$. We denote the root by $R_{*}(c)$. Note that at $\left(c, R_{*}(c)\right)$, we have $\Psi_{c}<0$ and $\Psi_{r}>0$, so by the implicit function theorem, $\frac{d}{d c} R_{*}(c)>0$. Thus, the inverse function of $R_{*}=R_{*}(c)$ exists and is given by (3.6). This completes the proof of Lemma 3.4.1.

Figure 1: A plot of $c_{*}(R)$

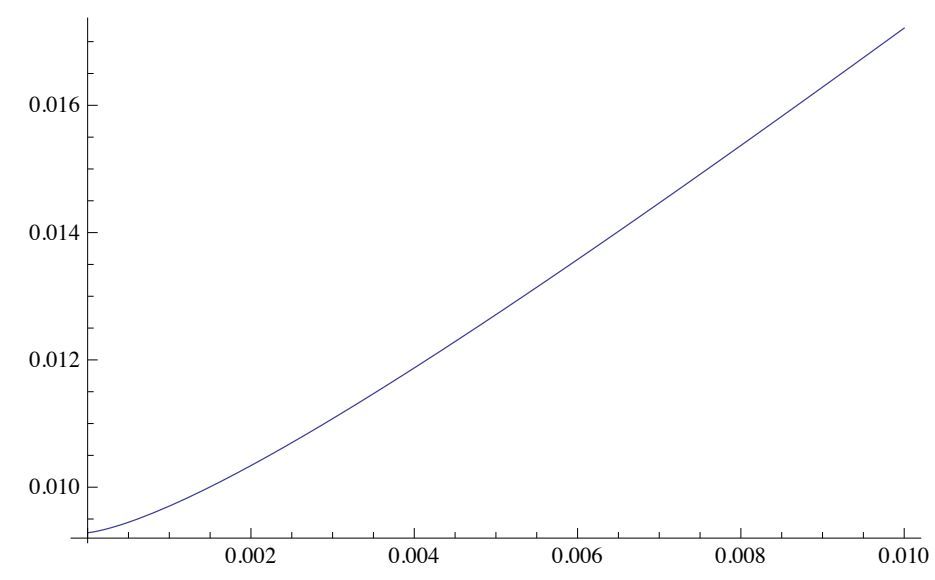




\subsection{THE SOLUTION OF THE INFINITE HORIZON PROBLEM}

Once we have the formula for $R_{*}$, we can derive the formula for $V_{*}$. We start with (3.3):

$$
\left\{V_{*}^{\prime}(u) G_{1}(u)-V_{*}(u) G_{1}^{\prime}(u)\right\} u^{b} e^{-\nu u}=\frac{2 c}{\sigma^{2}} \int_{u}^{\infty} \rho^{b-1} e^{-\nu \rho} G_{1}(\rho) d \rho \quad \forall u>R_{*} .
$$

Using the integrating factor $u^{-b} e^{\nu u} G_{1}^{-2}(u)$ we obtain for $R_{*}<r_{1}<r<\infty$,

$$
\frac{V_{*}(r)}{G_{1}(r)}=\frac{V_{*}\left(r_{1}\right)}{G_{1}\left(r_{1}\right)}+\frac{2 c}{\sigma^{2}} \int_{r_{1}}^{r} \frac{e^{\nu u}}{u^{b} G_{1}^{2}(u)} \int_{u}^{\infty} \rho^{b-1} e^{-\nu \rho} G_{1}(\rho) d \rho d u
$$

Integrating $\left(r^{b} e^{-\nu r} G_{1}^{\prime}\right)^{\prime}=2 \sigma^{-2} r^{b} e^{-\nu r} G_{1}$ gives $G^{\prime}(u)=-2 \sigma^{-2} u^{-b} e^{\nu u} \int_{u}^{\infty} \rho^{b} e^{-\nu \rho} G_{1}(\rho) d \rho$ so

$$
\begin{aligned}
& \frac{1}{G_{1}\left(r_{1}\right)}-\frac{1}{G_{1}(r)}=\int_{r_{1}}^{r} \frac{G_{1}^{\prime}(u)}{G_{1}^{2}(u)} d u=-\frac{2}{\sigma^{2}} \int_{r_{1}}^{r} \frac{e^{\nu u}}{u^{b} G_{1}^{2}(u)} \int_{u}^{\infty} \rho^{b} e^{-\nu \rho} G_{1}(\rho) d \rho d u, \\
& \begin{aligned}
\frac{V_{*}(r)-V_{*}\left(r_{1}\right)}{G_{1}(r)} & =\frac{V_{*}\left(r_{1}\right)}{G_{1}\left(r_{1}\right)}-\frac{V_{*}\left(r_{1}\right)}{G_{1}(r)}+\frac{2 c}{\sigma^{2}} \int_{r_{1}}^{r} \frac{e^{\nu u}}{u^{b} G_{1}^{2}(u)} \int_{u}^{\infty} \rho^{b-1} e^{-\nu \rho} G(\rho) d \rho d u \\
& =\frac{2}{\sigma^{2}} \int_{r_{1}}^{r} \frac{e^{\nu u}}{u^{b} G_{1}^{2}(u)} \int_{u}^{\infty}\left[\rho V\left(r_{1}\right)-c\right] \rho^{b-1} e^{-\nu \rho} G_{1}(\rho) d \rho d u .
\end{aligned}
\end{aligned}
$$

Now sending $r_{1} \searrow R_{*}$ we obtain the formula

$$
\begin{aligned}
V_{*}(r) & =V_{*}\left(R_{*}\right)-\frac{2 G_{1}(r)}{\sigma^{2}} \int_{R_{*}}^{r} \frac{e^{\nu u}}{u^{b} G_{1}^{2}(u)} \int_{u}^{\infty}\left[V\left(R_{*}\right) \rho-c\right] \rho^{b} e^{-\nu \rho} G_{1}(\rho) d \rho d u \\
& =V\left(R_{*}\right)-\frac{2 G_{1}(r)}{\sigma^{2}} \int_{R_{*}}^{r} \frac{e^{\nu u}}{u^{b} G_{1}^{2}(u)} \int_{R_{*}}^{u}\left[c-V\left(R_{*}\right) \rho\right] \rho^{b} e^{-\nu \rho} G_{1}(\rho) d \rho d u
\end{aligned}
$$

by (3.4). In the case $c>c^{*}$, we have $R_{*}>0$ and $V_{*}\left(R_{*}\right)=1$ so we obtain the formula

$$
V_{*}(r)= \begin{cases}1 & \text { if } r \in\left[0, R_{*}\right], \\ 1-\frac{2}{\sigma^{2}} \int_{R_{*}}^{r} \int_{R_{*}}^{u} \frac{[c-\rho] \rho^{b} e^{\nu u} G_{1}(\rho) G_{1}(r)}{u^{b} e^{\nu \rho} G_{1}^{2}(u)} d \rho d u & \text { if } r \geqslant R_{*} .\end{cases}
$$

In the case $c \in\left(0, c^{*}\right]$ we have $R_{*}=0$ and $V_{*}(0)=c / c^{*}$ so we obtain $V_{*}=c V_{* *} / c^{*}$ where

$$
V_{* *}(r)=1-\frac{2}{\sigma^{2}} \int_{0}^{r} \int_{0}^{u} \frac{\left[c^{*}-\rho\right] \rho^{b} e^{\nu u} G(\rho) G(r)}{u^{b} e^{\nu \rho} G^{2}(u)} d \rho d u \quad \forall r \geqslant 0 .
$$

So far we have shown that if $V_{*}$ is a weak solution of (3.1), then it must be given by the above formula with $R_{*}$ the solution of (3.4). 
Now we show that the function $V_{*}$ given above is indeed a solution of the variational inequality (3.1). Indeed, $V_{*}$ satisfies the differential equation $\mathcal{L} V_{*}+c=-0$ in $\left[R_{*}, \infty\right)$, and by L'Hôpital's rule, $\lim _{r \rightarrow \infty} V_{*}(r)=0$ so $V_{*}$ is bounded. In addition, by the definition of $\left(R_{*}, V\left(R_{*}\right)\right)$ in (3.4),

$$
\int_{R_{*}}^{u}\left[c-V\left(R_{*}\right) \rho\right] \rho^{b} e^{-\nu \rho} G(\rho) d \rho>0 \quad \forall u>R_{*},
$$

so $V_{*}<1$ on $\left(R_{*}, \infty\right)$. Furthermore, when $R_{*}>0$, we have in the interval $\left[0, R_{*}\right], V_{*} \equiv 1$ and $\mathcal{L} V_{*}=c-r \geqslant c-R_{*}>0$ for $r \in\left[0, R^{*}\right]$. Thus, $V_{*}$ is a bounded solution of the variational inequality (3.1). We summarize our analysis as follows:

Theorem 1. Let $\sigma, \kappa, \theta$ be given fixed positive constants and define $b, \nu, \eta, \mu, a$ by (2.2) and $G_{1}(\cdot)$ by Lemma 3.1.1. Set $c^{*}$ as in (3.5) and for $c \geqslant c^{*}$ let $R_{*}=R_{*}(c)$ be the inverse of the function given (3.6). For each $c>0$, problem (3.1) admits a unique solution and is given by

1. If $c \in\left(0, c^{*}\right]$, then $V_{*}<1$ in $(0, \infty)$ and $V_{*}=c V_{* *} / c^{*}$ with $V_{* *}$ given by (3.9);

2. If $c \in\left(c^{*}, \infty\right)$, then $V_{*}$ is given by (3.8) with $R_{*}=R_{*}(c)>0$.

In addition $V_{*}^{\prime}\left(R^{*}\right)=0>V_{*}^{\prime}(\cdot)$ in $\left(R^{*}, \infty\right)$ and $V_{*}(r)=c r^{-1}+c \kappa r^{-2}+O\left(r^{-3}\right)$ as $r \rightarrow \infty$. 


\subsection{THE VARIATIONAL APPROACH}

In this chapter, we solve the variational inequality (2.1). We use both classical technique for obstacle problems (e.g. [25]) and the modern notion of viscosity solutions (e.g. [16]).

\subsection{THE MAIN RESULT}

We begin with a definition of a solution to the variational inequality.

Definition 4.1.1. A solution of $(2.1)$ is a function $W \in C\left([0, \infty)^{2}\right)$ with the following properties:

1. $0 \leqslant W \leqslant M$ on $[0, \infty)^{2}, W(\cdot, 0) \equiv 0$ on $[0, \infty)$, and for $b:=2 \kappa \theta / \sigma^{2}$,

$$
\lim _{r \searrow 0} r^{b-1}\{W(r, \tau)-W(0, \tau)\}=0 \quad \forall \tau>0 ;
$$

2. $W \in C^{\infty}(Q)$ and $W_{\tau}-\mathcal{L} W+P=0$ in $Q:=\left\{(r, \tau) \in(0, \infty)^{2} \mid W(r, \tau)>0\right\}$;

3. If $\psi$ is a smooth function in $B:=\left(r_{0}-\delta, r_{0}+\delta\right) \times\left(\tau_{0}-\delta, \tau_{0}\right]$ for some positive $r_{0}, \tau_{0}$ and $\delta$ such that $\psi\left(r_{0}, \tau_{0}\right)=W\left(r_{0}, \tau_{0}\right)$ and $\psi \leqslant W$ in $B$, then

$$
\psi_{\tau}\left(r_{0}, \tau_{0}\right)-\mathcal{L} \psi\left(r_{0}, \tau_{0}\right)+P\left(r_{0}, \tau_{0}\right) \geqslant 0 .
$$

Remark 4.1.1. (i) The condition $W \leqslant M$, originating from $V \geqslant 0$, is equivalent to $W$ being bounded.

(ii) When $W_{r} \in C\left((0, \infty)^{2}\right)$, (4.1) can be written as $\lim _{r \searrow 0} r^{b} W_{r}(r, \tau)=0$. We call it the noflux boundary condition. In the case $b \geqslant 1$, the no-flux boundary condition (4.1) is automatically satisfied since $W$ is bounded. When $b \in(0,1)$, the probability that $r_{t}=0$ is positive and the CIR model implicitly assumes that $r_{t}$ immediately becomes positive whenever it becomes zero; a 
natural boundary condition associated with the Black-Scholes equation for value functions is the no-flux boundary condition. When the no-flux boundary condition is removed, uniqueness does not hold when $b \in(0,1)$ and $c$ is sufficiently small; this will be addressed in Section 5.6.

(iii) In earlier fashions, weak solutions of obstacle problems are defined in terms of a variational inequality in the sense of distributions [25]. Nevertheless, since viscosity solutions developed in the last two decades have been shown to be very convenient and natural for problems arising from mathematical finance, optimal control, game theory, etc., here we adopt the essence of the viscosity solution in property (3) of Definition 4.1.1, which is indeed the celebrated viscosity formulation of the differential inequality $W_{\tau}-\mathcal{L} W+P \geqslant 0$. Together with $W \geqslant 0$, this property provides a weak formulation of the smooth fit condition: $W_{r}=0$ at the free boundary $\Gamma:=\partial Q \cap(0, \infty)^{2}$.

For the remainder of this chapter, we shall prove the following:

Theorem 2. Problem (2.1) admits a unique solution. The solution satisfies the following:

$$
0 \leqslant W_{r} \leqslant \frac{1}{\kappa c}, \quad-c M \leqslant W_{\tau} \leqslant M^{\prime},\left|W_{r r}(r, \tau)\right| \leqslant \frac{2}{c \sigma^{2}}\left(1+\frac{c+\theta}{r}\right) .
$$

\subsection{UNIQUENESS}

Here we show that (2.1) admits at most one solution. Suppose, on the contrary, that there exist two different solutions, say $W_{1}$ and $W_{2}$. Then there exist $T>0$ and $r_{1} \geqslant 0$ such that $W_{2}\left(r_{1}, T\right) \neq$ $W_{1}\left(r_{1}, T\right)$. By continuity and exchanging the roles of $W_{2}$ and $W_{1}$ if necessary, we can assume that $r_{1}>0$ and $\delta:=\left[W_{2}\left(r_{1}, T\right)-W_{1}\left(r_{1}, T\right)\right] / 4>0$.

Let $G_{1}$ and $G_{2}$ be the functions given by Lemma 3.1.1 and set $G=G_{1}+G_{2}$. Then

$$
\mathcal{L} G=0<G \text { in }(0, \infty), \quad \lim _{r \rightarrow \infty} G(r)=\infty, \quad \lim _{r \searrow 0} r^{b} G_{r}=-1 .
$$

We claim that the following $\Phi$ on $(0, \infty) \times[0, T]$ attains a positive global maximum:

$$
\Phi(r, \tau):=W_{2}(r, \tau)-W_{1}(r, \tau)-\delta-\delta G(r) G\left(r_{1}\right)^{-1}
$$

Indeed, (i) $\Phi\left(r_{1}, T\right)>0$; (ii) $\Phi(\cdot, 0)<0$; (iii) as $r \rightarrow \infty, \Phi(r, \tau) \rightarrow-\infty$ uniformly in $\tau \in[0, T]$; and (iv) as $r \searrow 0$, one of the following holds: 
1. If $b \geqslant 1$, then $\lim _{r \searrow 0} G(r)=\infty$ so $\lim _{r \searrow 0} \Phi(r, \tau)=-\infty$ uniformly in $\tau \in[0, T]$;

2. If $b \in(0,1)$, then $\Phi \in C\left([0, \infty)^{2}\right)$, so that there exists $(\hat{r}, \hat{\tau}) \in[0,1] \times[0, T]$ such that $\Phi(\hat{r}, \hat{\tau})=\max _{\tau \in[0, T], r \in[0,1]} \Phi(r, \tau)$. We must have $\hat{r}>0$ since

$$
\lim _{r \searrow 0} r^{b-1}[\Phi(r, \hat{\tau})-\Phi(0, \hat{\tau})]=\lim _{r \searrow 0} \frac{\delta[G(0)-G(r)]}{r^{1-b} G\left(r_{1}\right)}=\frac{\delta}{(1-b) G\left(r_{1}\right)}>0
$$

Hence, there exists $\left(r_{0}, \tau_{0}\right) \in(0, \infty) \times(0, T]$ such that

$$
\Phi\left(r_{0}, \tau_{0}\right)=\sup _{(0, \infty) \times[0, T]} \Phi \geqslant \Phi\left(r_{1}, \tau_{1}\right)>0
$$

Note that $W_{2}\left(r_{0}, \tau_{0}\right)>W_{1}\left(r_{0}, \tau_{0}\right)+\Phi\left(r_{0}, \tau_{0}\right)>0$ so $W_{2}$ is smooth near $\left(r_{0}, \tau_{0}\right)$. Define

$$
\psi(r, \tau)=W_{2}(r, \tau)-\Phi\left(r_{0}, \tau_{0}\right)-\delta-\delta G(r) G^{-1}\left(r_{1}\right)
$$

Then $\psi$ is smooth near $\left(r_{0}, \tau_{0}\right), \psi-W_{1}=\Phi(r, \tau)-\Phi\left(r_{0}, \tau_{0}\right) \leqslant 0$ in $(0, \infty) \times[0, T]$, and $\psi\left(r_{0}, \tau_{0}\right)=W_{1}\left(r_{0}, \tau_{0}\right)$. Consequently, by property (3) of Definition 4.1.1 for $W_{1}$,

$$
0 \leqslant \psi_{\tau}\left(r_{0}, \tau_{0}\right)-\mathcal{L} \psi\left(r_{0}, \tau_{0}\right)+P\left(r_{0}, \tau_{0}\right)=-r_{0}\left[\Phi\left(r_{0}, \tau_{0}\right)+\delta\right]<0
$$

which is impossible. This contradiction shows that (2.1) admits at most one solution. 


\subsection{EXISTENCE}

To establish existence, we use the classic penalization technique for obstacle problems (e.g. [25]). Let $\rho(\cdot)$ be a smooth function on $\mathbb{R}$ that has the following properties:

$$
\rho(x)=0 \forall x \geqslant 1, \quad \rho^{\prime}(x) \leqslant 0 \forall x<1, \quad \rho(0)=1 .
$$

For $\varepsilon \in(0,1]$, consider the initial boundary value problem, for $W^{\varepsilon}=W^{\varepsilon}(r, \tau)$,

$$
\left\{\begin{array}{l}
\mathcal{A}^{\varepsilon}\left[W^{\varepsilon}\right]=0 \quad \text { in } I_{\varepsilon} \times(0, \infty) \\
W^{\varepsilon}=0 \text { on } \bar{I}_{\varepsilon} \times\{0\}, \quad W_{r}^{\varepsilon}=0 \text { on } \partial I_{\varepsilon} \times(0, \infty)
\end{array}\right.
$$

where $I_{\varepsilon}=\left(0, \varepsilon^{-1}\right), \mathcal{A}^{\varepsilon}[\phi]:=\phi_{\tau}-\mathcal{L} \phi+P-\varepsilon \sigma^{2} \phi_{r r}-\rho\left(\varepsilon^{-1} \phi\right)$.

Note that $\mathbf{0}$ is a sub-solution and $M(\cdot)$ is a super-solution since

$$
\begin{aligned}
\mathcal{A}^{\varepsilon}[\mathbf{0}] & =(c-r) M(\tau)-\rho(0) \leqslant c M(\tau)-1<0, \\
\mathcal{A}^{\varepsilon}[M] & =M^{\prime}+r M+(c-r) M-\rho\left(\varepsilon^{-1} M\right)=1-\rho \geqslant 0 .
\end{aligned}
$$

Hence, by the classical theory of parabolic equations [23], problem (4.3) on $\left[0, \varepsilon^{-1}\right] \times[0, \infty)$ is well-posed; it admits a unique classical solution and the solution satisfies $0 \leqslant W^{\varepsilon} \leqslant M$.

Next differentiating $\mathcal{A}^{\varepsilon}\left[W^{\varepsilon}\right]=0$ with respect to $r$ we obtain $\mathcal{L}_{1}^{\varepsilon}\left[W_{r}\right]=0$ where

$$
\mathcal{L}_{1}^{\varepsilon}[\phi]:=\phi_{\tau}-(\varepsilon+r / 2) \sigma^{2} \phi_{r r}+\left(\kappa r-\kappa \theta-\sigma^{2} / 2\right) \phi_{r}+\left(\kappa+r-\varepsilon^{-1} \rho^{\prime}\right) \phi+W^{\varepsilon}-M .
$$

Then $\mathbf{0}$ is a subsolution and $(\kappa c)^{-1} \mathbf{1}$ is a super-solution so $0 \leqslant W_{r}^{\varepsilon} \leqslant(\kappa c)^{-1}$.

Differentiating $\mathcal{A}^{\varepsilon}\left[W^{\varepsilon}\right]=0$ with respect to $\tau$ we obtain $\mathcal{L}_{2}^{\varepsilon}\left[W_{\tau}^{\varepsilon}\right]=0$ where

$$
\mathcal{L}_{2}^{\varepsilon}[\phi]=\phi_{\tau}-(\varepsilon+r / 2) \sigma^{2} \phi_{r r}+(\kappa r-\kappa \theta) \phi_{r}+\left(r-\varepsilon^{-1} \rho^{\prime}\right) \phi+(c-r) M^{\prime}
$$

Note that $\partial_{r} W_{\tau}^{\varepsilon}=0$ on $\partial I_{\varepsilon} \times[0, \infty)$ and $W_{\tau}^{\varepsilon}(\cdot, 0)=\rho(0)=1$. Then $M^{\prime}$ is a super-solution and $-c M$ is a subsolution, so $-c M \leqslant W_{\tau}^{\varepsilon} \leqslant M^{\prime}$. 
Finally, using the equation $\mathcal{A}^{\varepsilon}\left[W^{\varepsilon}\right]=0$ and estimates on $W^{\varepsilon}, W_{r}^{\varepsilon}$ and $W_{\tau}^{\varepsilon}$ we derive that

$$
\begin{aligned}
\left|W_{r r}^{\varepsilon}\right| & =\frac{2}{\sigma^{2}[r+2 \varepsilon]}\left|\left[W_{\tau}^{\varepsilon}+c M-\rho\right]-\kappa \theta W_{r}+r\left[\kappa W_{r}^{\varepsilon}+W^{\varepsilon}-M\right]\right| \\
& \leqslant \frac{2}{\sigma^{2} r}\left(1+\frac{\theta}{c}+\frac{r}{c}\right)=\frac{2}{c \sigma^{2}}\left(1+\frac{c+\theta}{r}\right) .
\end{aligned}
$$

The above estimates allow us to find a sequence $\left\{\varepsilon_{j}\right\}_{j=1}^{\infty}$ satisfying $\varepsilon_{j} \searrow 0$ as $j \rightarrow \infty$ and functions $W$ and $\xi$ defined on $D:=(0, \infty) \times[0, \infty)$ such that as $j \rightarrow \infty$,

$$
\begin{gathered}
W^{\varepsilon_{j}} \longrightarrow W \quad \text { in } C^{2 \alpha, \alpha}\left(\left[R^{-1}, R\right] \times[0, R]\right) \forall R>1, \alpha \in(0,1), \\
\left(W_{\tau}^{\varepsilon_{j}}, W_{r r}^{\varepsilon_{j}}, \rho\left(\varepsilon_{j}^{-1} W^{\varepsilon_{j}}\right)\right) \longrightarrow\left(W_{\tau}, W_{r r}, \xi\right) \text { weakly in }\left(L_{\mathrm{loc}}^{p}(D)\right)^{3} \forall p>1 .
\end{gathered}
$$

In addition, $W_{\tau}-\mathcal{L} W+P=\xi$ in $L^{\infty}\left((0, \infty)^{2}\right)$ and $0 \leqslant \xi \leqslant 1$.

If $\left(r_{0}, \tau_{0}\right) \in Q:=\left\{(r, \tau) \in(0, \infty)^{2} \mid W(r, \tau)>0\right\}$ which is an open set, then by continuity and uniform convergence, there exists $\delta>0$ such that $W^{\varepsilon_{j}}>\delta$ and therefore $\rho\left(\varepsilon_{j}^{-1} W\right)=0$ in $B=\left[r_{0}-\delta, r_{0}+\delta\right] \times\left[\tau_{0}-\delta, \tau_{0}+\delta\right]$ for every integer $j \gg 1$. This implies that $W$ is smooth and $\xi \equiv 0$ in $B$. Hence, $W \in C^{\infty}(Q)$ and $\min \{\xi, W\}=0$.

If $\psi$ and $\left(r_{0}, \tau_{0}, \delta\right)$ satisfy the assumptions in property (3) of Definition 4.1.1, comparing $W^{\varepsilon}$ with $\psi_{\varepsilon}(r, \tau)=\psi(r, \tau)-\left(\tau-\tau_{0}\right)^{2}-\left(r-r_{0}\right)^{4}-c_{\varepsilon}$ where $c_{\varepsilon}$ is a constant such that the maximum of $\psi_{\varepsilon}-W^{\varepsilon}$ in $B$ is zero, evaluating the differential initial inequality at the point of maximum and using $\rho\left(\varepsilon^{-1} W^{\varepsilon}\right) \geqslant 0$, we obtain (4.2) after sending $\varepsilon=\varepsilon_{j} \searrow 0$.

Finally, as $W$ is Lipschitz continuous, it also satisfies the no-flux boundary condition (4.1). Hence, $W$ is a solution. This completes the proof of Theorem 2. 


\subsection{THE FREE BOUNDARY APPROACH}

A long and rich connection exists between contracts with early termination, such as Americanstyle options, and free boundary problems. Predating the celebrated 1973 Black-Scholes paper[3], McKean [34] established a connection between the American put and the Stefan free boundary problem.

Since the emergence of the Black-Scholes model, the relationship between American options and free boundary problems has been well developed $[4,7]$. This relationship has been extended to encompass the broader topic of optimal stopping problems [37].

Recently, rigorous analytical and numerical results for the free boundary problem formulation of American puts have been developed by Xinfu Chen and John Chadam [9, 10, 11, 12, 13]. By using new and classical techniques [16, 25], they have been able to settle a variety of issues regarding convexity of the optimal exercise boundary in addition to providing accurate numerical approximations of the optimal exercise boundary using integral equation methods. With Dejun Xie, they were able extend the integral equation method developed for American puts to the case of mortgage prepayment under the Vasicek model [39].

In this chapter, we follow the ideas of [30] and study (2.1) with a free boundary approach. Such an approach works because the solution possesses many special monotonicity properties. By using this approach, we are able to establish the smoothness of the free boundary.

\subsection{THE FREE BOUNDARY PROBLEM}

We begin with establishing that the free boundary is a well-defined set. To this end, notice that if $W\left(r_{0}, \tau_{0}\right)=0$, then setting $\psi \equiv 0$ in (4.2) gives $r_{0} \leqslant c$. Thus, the following function is 
well-defined:

$$
x(\tau):=\inf \{r>0 \mid W(r, t)>0\} \quad \forall \tau>0 .
$$

The monotonicity $W_{r} \geqslant 0$ ensures that $W(r, \tau)>0$ if $r>x(\tau)$ and $W(r, \tau)=0$ if $r \in(0, x(\tau))$. Thus, we can define the free boundary by

$$
\Gamma:=\{(r, \tau) \mid \tau>0, r=x(\tau)>0\}
$$

The obstacle problem (2.1) can now be formulated as a free boundary problem as follows:

Find $x \in C((0, \infty))$ and $W \in C(\bar{Q})$ with $Q=\{(r, \tau) \mid \tau>0, r>x(\tau)\}$ such that $x \geqslant 0$ and

$$
\begin{cases}W_{\tau}-\mathcal{L} W+P=0<W & \text { in } Q, \\ W(x(\tau), \tau)=0, \quad W_{r}(x(\tau), \tau)=0 & \text { if } \tau>0, x(\tau)>0, \\ \lim _{r \searrow 0} r^{b} W_{r}(r, \tau)=0 & \text { if } \tau>0, x(\tau)=0, \\ W(r, 0)=0 & \forall r \geqslant 0 .\end{cases}
$$

Remark 5.1.1. (i) As a default, the solution of (5.2) is first extended to $(0, \infty)^{2}$ by $W(r, \tau)=0$ for $r \in(0, x(\tau))$ and then extended to $[0, \infty)^{2}$ by $W(0, \tau)=\lim _{r \searrow 0} W(r, \tau)$.

(ii) Clearly, the solution $W$ of (2.1), together with $x$ defined in (5.1), is a solution of (5.2). This is typically how the free boundary problem (5.2) is solved. The advantage of using (5.2) is that $C^{1}$ regularity of $x$ implies $C^{\infty}$ regularity of $x$, by a hodograph transformation [25].

To establish the $C^{1}$ regularity of the free boundary, we follow the ideas in [30] converting (5.2) into a Stefan type free boundary problem. We work on the function

$$
u:=W_{\tau}+c W .
$$

Using $M^{\prime}+c M=1$ one finds that $\partial_{\tau} u-\mathcal{L} u=r-c$ in $Q$. To derive free boundary conditions for $u$, we differentiate (formally) the two free boundary conditions in the second line of (5.2) to obtain

$$
W_{\tau}(x(\tau), \tau)=0, \quad W_{r r}(x(\tau), \tau) x^{\prime}(\tau)+W_{r \tau}(x(\tau), \tau)=0
$$


Here and in the sequel, all derivatives on the free boundary are right-hand side derivatives. Hence, $u(x(\tau), \tau)=0$ and $u_{r}(x, \tau)=W_{\tau r}(x, \tau)=-W_{r r}(x, \tau) x^{\prime}$. The quantity $W_{r r}(x, \tau)$ can be evaluated by using the equation $W_{\tau}-\mathcal{L} W+P=0$ for $r>x(\tau)$, which gives

$$
W_{r r}(x, \tau)=\frac{2 P(x, \tau)}{\sigma^{2} x}=\frac{2(c-x)\left(1-e^{-c \tau}\right)}{c \sigma^{2} x} .
$$

One can derive that $x(0)=c$, so formally, (5.2) can be reformulated as follows:

Find $(u, x)$ such that $x(\cdot) \geqslant 0, u \in C(\bar{Q})$ with $Q=\{(r, \tau) \mid \tau>0, r>x(\tau))\}$, and

$$
\begin{cases}u_{\tau}-\mathcal{L} u=r-c & \forall r>x(\tau), \tau>0, \\ u(x(\tau), \tau)=0 & \text { when } \tau>0, x(\tau)>0, \\ \frac{d x}{d \tau}=-\frac{c \sigma^{2} x u_{r}(x, \tau)}{2\left[1-e^{-c \tau}\right][c-x]} & \text { when } \tau>0, x(\tau)>0, \\ \lim _{r \searrow 0} r^{b} u_{r}(r, \tau)=0 & \text { when } \tau>0, x(\tau)=0, \\ x(0)=c, u(\cdot, 0)=0 . & \end{cases}
$$

The free boundary problem (5.4) is a Stefan type free boundary problem. It would admit a classical solution [24, 29] if the third equation in (5.4) were not singular at $\tau=0$ and the elliptic operator $\mathcal{L}$ were not degenerate at $r=0$. In the rest of this section we solve (5.4) using the ideas in [30], i.e., taking the limit of a sequence of regular Stefan problems that approximate (5.4).

\subsection{REGULARIZED PROBLEM}

We regularize (5.4) by the following: for $0<\varepsilon \ll 1$,

$$
\begin{cases}\partial_{\tau} u^{\varepsilon}-\mathcal{L} u^{\varepsilon}=r-c & \forall \tau \in\left(0, T_{\varepsilon}\right), r>x^{\varepsilon}(\tau), \\ u^{\varepsilon}\left(x^{\varepsilon}(\tau), \tau\right)=0, & \forall \tau \in\left(0, T_{\varepsilon}\right), \\ x^{\varepsilon \prime}=-\ell^{\varepsilon}\left(x^{\varepsilon}, \tau\right) u_{r}^{\varepsilon}\left(x^{\varepsilon}, \tau\right) & \forall \tau \in\left(0, T_{\varepsilon}\right), \\ x^{\varepsilon}(0)=c, \quad u^{\varepsilon}(r, 0)=0 & \forall r \geqslant c\end{cases}
$$


where

$$
\ell^{\varepsilon}(r, \tau):=\frac{(r-\varepsilon) c \sigma^{2}}{2\left[1-e^{-c \tau}\right][c-r]+4 \varepsilon} .
$$

The function $\ell^{\varepsilon}$ is smooth on $(-\infty, c] \times[0, \infty)$. The factor $r-\varepsilon$ on the numerator guarantees that the solution of the third equation in (5.5) satisfies $x^{\varepsilon}>\varepsilon$. The unboundedness of the spatial domain and operator $\mathcal{L}$ at $r=\infty$ is resolved by restricting the solution to be bounded. Following [30], we shall prove the following:

Lemma 5.2.1. Let $\varepsilon_{0}=\min \left\{c, c^{-1}\right\}$ and $K_{1}=\max \left\{b, c \kappa^{-1}\right\}$. For each $\varepsilon \in\left(0, \varepsilon_{0}\right)$, problem (5.5) with $T^{\varepsilon}=\infty$ admits a solution satisfying $x^{\varepsilon} \in C^{2}([0, \infty)) \cap C^{\infty}((0, \infty))$ and $u^{\varepsilon} \in C^{3,3 / 2}\left(\bar{Q}_{\varepsilon}\right) \cap$ $C^{\infty}\left(\bar{Q}_{\varepsilon} \backslash(c, 0)\right)$ where $Q_{\varepsilon}:=\left\{(r, \tau) \mid \tau>0, r>x^{\varepsilon}(\tau)\right\}$. In addition, for $\tau>0$ and $r>x^{\varepsilon}(\tau)$,

$$
x^{\varepsilon^{\prime}}<0, \quad \varepsilon<x^{\varepsilon}<c, \quad u_{\tau}^{\varepsilon}>0, \quad 0<u^{\varepsilon}<1, \quad 0<x^{\varepsilon} u_{r}^{\varepsilon}<K_{1} .
$$

Proof. 1. Local in Time Existence. When $T^{\varepsilon}$ is small, the existence of a solution follows by the fixed point of a contract mapping defined as follows. Fix $\alpha \in(0,1)$ and define

$$
\mathbf{X}:=\left\{x \in C^{\frac{3+\alpha}{2}}\left(\left[0, T^{\varepsilon}\right]\right) \mid x(0)=c, \varepsilon \leqslant x \leqslant c+\varepsilon, \quad\left\|x^{\prime}\right\|_{C^{\frac{1+\alpha}{2}}\left(\left[0, T^{\varepsilon}\right]\right)} \leqslant 1\right\} .
$$

For $x \in \mathbf{X}$ define $u$ as the solution of $u_{\tau}=\mathcal{L} u+r-c$ in $D:=\left\{(r, \tau) \mid r>x(\tau), 0<\tau \leqslant T^{\varepsilon}\right\}$ with $u=0$ on the parabolic boundary of $D$. There holds the first order compatibility condition (the equation $u_{\tau}=\mathcal{L} u+r-c$ at the corner $(x(0), 0)$ with the left-hand side evaluated by the boundary value and the right-hand by the initial value): $0=x(0)-c$. Hence, $u \in C^{3+\alpha, \frac{3+\alpha}{2}}(\bar{D})$ and $u_{r} \in C^{2+\alpha, 1+\frac{\alpha}{2}}(\bar{D})$. Define $\mathbf{T}[x]=\hat{x}$ by, for $\tau \in\left[0, T^{\varepsilon}\right]$,

$$
\hat{x}(\tau)=\varepsilon+(c-\varepsilon) \exp \left(-\int_{0}^{\tau} \frac{c \sigma^{2} u_{r}(x(t), t)}{2\left[1-e^{-c t}\right][c-x(t)]+4 \varepsilon} d t\right) .
$$

Then $\hat{x}(0)=c, \hat{x} \in C^{2+\alpha / 2}\left(\left[0, T^{\varepsilon}\right]\right)$ and

$$
\frac{d \hat{x}}{d \tau}=-\frac{(\hat{x}-\varepsilon) c \sigma^{2}}{2\left[1-e^{-c \tau}\right][c-x]+4 \varepsilon} u_{r}(x, \tau) .
$$

Since $u_{r}(c, 0)=0$, one can show that when $T^{\varepsilon}$ is sufficiently small, $\mathbf{T}$ is a contraction and admits a fixed point in $\mathbf{X}$, which provides a solution of (5.5), with $u^{\varepsilon} \in C^{3+\alpha,(3+\alpha) / 2}\left(\overline{Q_{\varepsilon}\left(T^{\varepsilon}\right)}\right)$ and $x^{\varepsilon} \in$ $C^{2+\alpha / 2}\left(\left[0, T^{\varepsilon}\right]\right)$ where $\left.Q_{\varepsilon}\left(T^{\varepsilon}\right)=\left\{(r, \tau) \mid r>x^{\varepsilon}(\tau), 0<\tau<T^{\varepsilon}\right)\right\}$. By a bootstrap argument one derives that $x^{\varepsilon} \in C^{\infty}\left(\left(0, T^{\varepsilon}\right]\right)$ and $u^{\varepsilon} \in C^{\infty}\left(\overline{Q_{\varepsilon}\left(T^{\varepsilon}\right)} \backslash\{(c, 0)\}\right)$. 
2. Monotonicity of the Free Boundary. Let $\left[0, T^{\varepsilon}\right), 0<T^{\varepsilon}<\infty$, be an existence interval of a classical solution of (5.5) satisfying $\varepsilon<x^{\varepsilon} \leqslant c+\varepsilon$. Here we show that $x^{\varepsilon \prime} \leqslant 0$ in $\left[0, T^{\varepsilon}\right)$.

Consider the function $v^{\varepsilon}:=u_{\tau}^{\varepsilon}$. We have $v_{\tau}^{\varepsilon}-\mathcal{L} v^{\varepsilon}=0$ in $Q_{\varepsilon}\left(T^{\varepsilon}\right)$ and

$$
v^{\varepsilon}(r, 0)=u_{\tau}^{\varepsilon}(r, 0)=\mathcal{L} u^{\varepsilon}(r, 0)+r-c=r-c \geqslant 0 \quad \forall r \geqslant c=x^{\varepsilon}(0) .
$$

On the free boundary, differentiating $u^{\varepsilon}\left(x^{\varepsilon}(\tau), \tau\right)=0$ gives $u_{r}^{\varepsilon}\left(x^{\varepsilon}, \tau\right) x^{\varepsilon \prime}+u_{\tau}^{\varepsilon}\left(x^{\varepsilon}, \tau\right)=0$, so that

$$
u_{\tau}^{\varepsilon}\left(x^{\varepsilon}, \tau\right)=-u_{r}^{\varepsilon}\left(x^{\varepsilon}, \tau\right) x^{\varepsilon \prime}=\ell^{\varepsilon}\left(x^{\varepsilon}, \tau\right)\left[u_{r}^{\varepsilon}\left(x^{\varepsilon}, \tau\right)\right]^{2} \geqslant 0 .
$$

Consequently, by the maximum principle, we obtain $v^{\varepsilon}=u_{\tau}^{\varepsilon} \geqslant 0$ in $Q^{\varepsilon}\left(T^{\varepsilon}\right)$.

Since $u^{\varepsilon} \in C^{3+\alpha,(3+\alpha) / 2}\left(\overline{Q^{\varepsilon}\left(T^{\varepsilon} / 2\right)}\right)$, we have $u_{r \tau}^{\varepsilon} \in C^{\alpha, \alpha / 2}\left(\overline{Q_{\varepsilon}\left(T^{\varepsilon} / 2\right)}\right)$. Hence, $u_{\tau r}(c, 0)=1$, $x^{\varepsilon^{\prime}}(0)=-\ell^{\varepsilon}(c, 0) u_{r}^{\varepsilon}(c, 0)=0$ and $x^{\varepsilon \prime \prime}(0)=-\ell^{\varepsilon}(c, 0) u_{r \tau}^{\varepsilon}(c, 0)=-\ell^{\varepsilon}(c, 0)<0$.

We claim that $x^{\varepsilon \prime}<0$ in $\left(0, T^{\varepsilon}\right)$. Suppose the assertion is not true. Then since $x^{\varepsilon \prime}(0)=0>$ $x^{\varepsilon \prime \prime}(0)$, there exists $\tau_{0} \in\left(0, T^{\varepsilon}\right)$ such that $x^{\varepsilon \prime}\left(\tau_{0}\right)=0$ and $x^{\varepsilon \prime}(\tau)<0$ for all $\tau \in\left(0, \tau_{0}\right)$. Then

$$
v^{\varepsilon}\left(x^{\varepsilon}\left(\tau_{0}\right), \tau_{0}\right)=u_{\tau}^{\varepsilon}\left(x^{\varepsilon}\left(\tau_{0}\right), \tau_{0}\right)=-u_{r}\left(x^{\varepsilon}\left(\tau_{0}\right), \tau_{0}\right) x^{\varepsilon \prime}\left(\tau_{0}\right)=0 .
$$

Since $v^{\varepsilon} \geqslant 0$, this implies that $\left(x^{\varepsilon}\left(\tau_{0}\right), \tau_{0}\right)$ is a point of global minimum of $v^{\varepsilon}$, so by Hopf's Lemma, $v_{r}^{\varepsilon}\left(x^{\varepsilon}\left(\tau_{0}\right), \tau_{0}\right)>0$. However, this leads to, since $x^{\varepsilon \prime}=-\ell^{\varepsilon} u_{r}^{\varepsilon}$ and $x^{\varepsilon \prime}\left(\tau_{0}\right)=0$, that

$$
x^{\varepsilon \prime \prime}\left(\tau_{0}\right)=-\ell^{\varepsilon}\left(x^{\varepsilon}\left(\tau_{0}\right), \tau_{0}\right) v_{r}^{\varepsilon}\left(x^{\varepsilon}\left(\tau_{0}\right), \tau_{0}\right)<0,
$$

contradicting the fact that $x^{\varepsilon^{\prime}}(\tau)<0$ in $\left(0, \tau_{0}\right)$ and $x^{\varepsilon^{\prime}}\left(\tau_{0}\right)=0$. Hence, $x^{\varepsilon \prime}<0$ in $\left(0, T^{\varepsilon}\right)$.

Remark 5.2.2. In [30], only $C^{2+\alpha, 1+\alpha / 2}$ regularity for $u^{\varepsilon}$ is established. The $C^{3+\alpha,(3+\alpha) / 2}$ regularity established here allows us to compute $x^{\varepsilon \prime \prime}(0)$ and therefore made the proof simpler. 
3. Certain A Priori Bounds. Since $u^{\varepsilon}=0$ on the parabolic boundary and $u_{\tau}^{\varepsilon}=v^{\varepsilon} \geqslant 0$ and $x^{\varepsilon \prime} \leqslant 0$, we have $u^{\varepsilon} \geqslant 0$. Comparing $u^{\varepsilon}$ with 1 we obtain $u^{\varepsilon} \leqslant 1$. Hence, $0<u^{\varepsilon}<1$ in $Q_{\varepsilon}\left(T^{\varepsilon}\right)$.

Next we estimate $u_{r}^{\varepsilon}\left(x^{\varepsilon}\left(\tau_{0}\right), \tau_{0}\right)$ for an arbitrarily fixed $\tau_{0} \in\left(0, T^{\varepsilon}\right)$. Set $r_{0}=x^{\varepsilon}\left(\tau_{0}\right)$. For a constant $\beta>0$ to be determined, we compare $u^{\varepsilon}$ with $\bar{u}:=1-e^{\left(r_{0}-r\right) \beta}$ in $\overline{Q_{\varepsilon}\left(\tau_{0}\right)}$. Clearly $u^{\varepsilon}=0 \leqslant \bar{u}$ on the parabolic boundary of $Q_{\varepsilon}\left(\tau_{0}\right)$ since $x^{\varepsilon}(\tau)>r_{0}$ when $\tau \in\left[0, \tau_{0}\right)$. Also,

$$
\bar{u}_{\tau}-\mathcal{L}^{\varepsilon} \bar{u}+(c-r)=e^{\left(r_{0}-r\right) \beta}\left\{\beta\left(\sigma^{2} r \beta / 2-\kappa \theta\right)+(\kappa \beta-1) r\right]+c .
$$

Thus, $\bar{u}$ is a super-solution if we set $K_{1}:=\max \left\{b, c \kappa^{-1}\right\}$ and

$$
\beta:=\frac{K_{1}}{r_{0}}=\max \left\{\frac{2 \kappa \theta}{\sigma^{2} r_{0}}, \frac{c}{\kappa r_{0}}\right\} \geqslant \max \left\{\frac{2 \kappa \theta}{\sigma^{2} r}, \frac{1}{\kappa}\right\} \quad \forall r \geqslant x^{\varepsilon}(\tau), \tau \in\left(0, \tau_{0}\right] .
$$

Thus $u^{\varepsilon} \leqslant \underline{u}$ on $\overline{Q_{\varepsilon}\left(\tau_{0}\right)}$. Since $u^{\varepsilon}\left(r_{0}, \tau_{0}\right)=0=\underline{u}\left(r_{0}, \tau_{0}\right)$ we find that

$$
u_{r}^{\varepsilon}\left(r_{0}, \tau_{0}\right) \leqslant \underline{u}_{r}\left(r_{0}, \tau_{0}\right)=\beta=K_{1} r_{0}^{-1} .
$$

As $\tau_{0}$ is arbitrary, we conclude that $u_{r}^{\varepsilon}\left(x^{\varepsilon}(\tau), \tau\right) \leqslant K_{1} x_{\varepsilon}(\tau)^{-1} \leqslant K_{1} \varepsilon^{-1}$ on $\left[0, T^{\varepsilon}\right)$. Thus,

$$
\begin{aligned}
0 \leqslant-\frac{d x^{\varepsilon}(\tau)}{d \tau} & \leqslant \frac{c \sigma^{2} K_{1}}{2\left[1-e^{-c \tau}\right]\left[c-x^{\varepsilon}\right]+4 \varepsilon} \frac{x^{\varepsilon}-\varepsilon}{x^{\varepsilon}}, \\
c \geqslant x^{\varepsilon}(t) & \geqslant \varepsilon+(c-\varepsilon) e^{-c \sigma^{2} K_{1} \tau /\left(4 \varepsilon^{2}\right)} \quad \forall \tau \in\left[0, T^{\varepsilon}\right) .
\end{aligned}
$$

The uniform bounds (5.6) and (5.7) and a bootstrap argument for higher order regularity shows that the time interval of maximal existence of solution of (5.5) is both open and closed, so $T^{\varepsilon}=\infty$.

Finally we estimate $u_{r}^{\varepsilon}$. Differentiating $u_{\tau}^{\varepsilon}-\mathcal{L} u^{\varepsilon}+c-r=0$ we obtain $\mathcal{L}_{3}^{\varepsilon}\left[u_{r}^{\varepsilon}\right]=0$ where

$$
\mathcal{L}_{3}^{\varepsilon}[\phi]=\phi_{\tau}-\mathcal{L} \phi-\frac{\sigma^{2}}{2} \phi_{r}+\kappa \phi+u^{\varepsilon}-1 .
$$

Since $u^{\varepsilon}<1$, one can check that 0 is a subsolution so $u_{r}^{\varepsilon}>0$ in $Q_{\varepsilon}$. To establish upper bound, for an arbitrary $\tau_{0}>0$ we take $\beta=K_{1} x^{\varepsilon}\left(\tau_{0}\right)^{-1}$ and compare $u_{r}^{\varepsilon}$ with $\left(1-u^{\varepsilon}\right) \beta$ in $Q_{\varepsilon}\left(\tau_{0}\right)$. Note that $u^{\varepsilon} \leqslant \beta=\left[1-u^{\varepsilon}\right] \beta$ on the parabolic boundary of $Q_{\varepsilon}\left(\tau_{0}\right)$ and

$$
\begin{aligned}
\mathcal{L}_{3}^{\varepsilon}\left[\left(1-u^{\varepsilon}\right) \beta\right] & =\left[-u_{\tau}^{\varepsilon}+\mathcal{L} u^{\varepsilon}+r\right] \beta+\sigma^{2} u_{r}^{\varepsilon} \beta / 2+\kappa\left[1-u^{\varepsilon}\right] \beta+u^{\varepsilon}-1 \\
& =c \beta+\sigma^{2} \beta u_{r}^{\varepsilon} / 2+\left[1-u^{\varepsilon}\right](\kappa \beta-1) \geqslant 0 .
\end{aligned}
$$

Hence, by comparison, $u_{r}^{\varepsilon} \leqslant\left(1-u^{\varepsilon}\right) \beta$ on $\overline{Q_{\varepsilon}\left(\tau_{0}\right)}$, in particular, $u_{r}^{\varepsilon}\left(\cdot, \tau_{0}\right)<\beta=K_{1} / x^{\varepsilon}\left(\tau_{0}\right)$ on $\left(x^{\varepsilon}\left(\tau_{0}\right), \infty\right)$. This completes the proof of Lemma 5.2.1. 


\subsection{SMALL TIME BEHAVIOR}

To treat the singular point $(c, 0)$, we begin with the following necessary result.

Lemma 5.3.1. For each $\varepsilon \in\left(0, \varepsilon_{0}\right)$ let $\left(u^{\varepsilon}, x^{\varepsilon}\right)$ be the solution of (5.5). Define

$$
x^{*}(\tau):=\varlimsup_{\varepsilon \searrow 0} x^{\varepsilon}(\tau), \quad x_{*}(\tau):=\varliminf_{\varepsilon \searrow 0} x^{\varepsilon}(\tau) \quad \forall \tau>0 .
$$

Then both $x^{*}$ and $x_{*}$ are non-increasing functions on $(0, \infty)$ and

$$
x_{*}(\tau) \leqslant x^{*}(\tau)<c \quad \forall \tau>0, \quad \lim _{\tau \searrow 0} x_{*}(\tau)=c
$$

Proof. The following proof is almost the same as that for Lemmas 5.1 and 5.3 in [30]. Here we need only show that (i) $x^{*}(\tau)<c$ for every $\tau>0$ and (ii) $\lim _{\tau \searrow 0} x_{*}(\tau)=c$.

(i) Suppose on the contrary $x^{*}\left(\tau_{0}\right)=c$ for some $\tau_{0}>0$. Then along a sequence of $\varepsilon_{j} \searrow 0$, $x^{\varepsilon_{j}}\left(\tau_{0}\right) \rightarrow x^{*}\left(\tau_{0}\right)=c$. By monotonicity, $x^{\varepsilon_{j}}(\cdot) \rightarrow c$ uniformly on $\left[0, \tau_{0}\right]$. Also on $[c, \infty) \times\left[0, \tau_{0}\right]$, the family $\left\{u^{\varepsilon_{j}}\right\}$ approaches the unique bounded solution $u$ of

$$
u_{\tau}-\mathcal{L} u=r-c \text { in }(c, \infty) \times\left[0, \tau_{0}\right], \quad u=0 \text { on }\{0\} \times\left[0, \tau_{0}\right] \cup[0, \infty) \times\{0\} .
$$

Here $u=0$ on $\{0\} \times\left[0, \tau_{0}\right]$ is obtained from $u^{\varepsilon}\left(x^{\varepsilon}, \tau\right)=0$ and $0 \leqslant u_{r}^{\varepsilon} \leqslant K_{1} / x^{\varepsilon}\left(\tau_{0}\right)$. By the maximum principle, we have $u>0$ in $(c, \infty) \times\left(0, \tau_{0}\right]$ so $u_{r}>0$ on $\{0\} \times\left(0, \tau_{0}\right]$.

On the other hand, for any smooth function $\zeta$ that is compactly supported in $(0, \infty) \times\left(0, \tau_{0}\right)$, integrating $\left[u_{\tau}^{\varepsilon}-\mathcal{L} u^{\varepsilon}+(c-r)\right] \zeta=0$ over $Q_{\varepsilon}\left(\tau_{0}\right)$ we obtain, after integration by parts,

$$
\begin{aligned}
& \iint_{Q_{\varepsilon}\left(\tau_{0}\right)}\left\{-u^{\varepsilon} \zeta_{\tau}+\frac{\sigma^{2}}{2} u_{r}^{\varepsilon}[r \zeta]_{r}+\left[r u^{\varepsilon}-\kappa(\theta-r) u_{r}^{\varepsilon}+c-r\right] \zeta\right\} d r d \tau \\
= & -\int_{0}^{\tau_{0}} \frac{\sigma^{2}}{2} u_{r}^{\varepsilon}\left(x^{\varepsilon}, \tau\right) x^{\varepsilon} \zeta\left(x^{\varepsilon}, \tau\right) d \tau=O(1) \int_{0}^{\tau_{0}}\left|x^{\varepsilon \prime}(\tau)\right| d \tau=O(1)\left|x^{\varepsilon}\left(\tau_{0}\right)-c\right| .
\end{aligned}
$$

Thus, sending $\varepsilon \searrow 0$ along the sequence $\left\{\varepsilon_{j}\right\}$ we obtain

$$
\int_{0}^{\tau_{0}} \int_{c}^{\infty}\left\{-u \zeta_{\tau}+\frac{\sigma^{2}}{2} u_{r}[r \zeta]_{r}+\left[r u-\kappa(\theta-r) u_{r}+c-r\right] \zeta\right\} d r d \tau=0
$$

Integrating by parts for the first two terms and using the equation $u_{\tau}-\mathcal{L} u+c-r=0$, we obtain $\int_{0}^{\tau_{0}} u_{r}(c, \tau) \zeta(c, \tau) d \tau=0$. As $\zeta$ is an arbitrary test function, we find that $u_{r}(c, \cdot)=0$ on $\left(0, \tau_{0}\right)$, contradicting $u_{r}(c, \cdot)>0$ on $\left(0, \tau_{0}\right]$. This contradiction shows that $x^{*}(\tau)<c$ for every $\tau>0$. 
(ii) Suppose on the contrary that $\hat{c}:=\lim _{\tau \searrow 0} x_{*}(\tau)<c$. Set $\delta=(c-\hat{c}) / 4$. Then there exist sequences $\left\{\varepsilon_{j}\right\}$ and $\left\{\tau_{j}\right\}$ such that for each positive integer $j, 0<\tau_{j}<1 / j, x_{*}\left(\tau_{j}\right) \leqslant \hat{c}+\delta$, $0<\varepsilon_{j}<1 / j$, and $x^{\varepsilon_{j}}\left(\tau_{j}\right) \leqslant x_{*}\left(\tau_{j}\right)+\delta$. This implies that $x^{\varepsilon_{j}}\left(\tau_{j}\right) \leqslant \hat{c}+2 \delta=c-2 \delta$. Upon selecting a further subsequence, $\left\{u^{\varepsilon_{j}}\right\}$ approaches a limit that satisfies

$$
u_{\tau}-\mathcal{L} u+c-r=0 \leqslant u \text { in }(c-2 \delta, \infty) \times(0, \infty) .
$$

The limit is continuous on $(c, \infty) \times[0, \infty)$ and $u(r, 0)=0$ for $r>c$. Using $u_{r}^{\varepsilon} \geqslant 0$ and $u \geqslant 0$, we can also show that $u$ is continuous on $[c-2 \delta, c] \times\{0\}$ and $u(r, 0)=0$ for all $r \geqslant c-2 \delta$. Hence, $u \in C^{\infty}((c-2 \delta, \infty) \times[0, \infty))$. Direct calculation gives $u_{\tau}(r, 0)=\mathcal{L} u(r, 0)+r-c=r-c$ for all $r>c-2 \delta$. This implies that $u_{\tau}(c-\delta, 0)=-\delta<0$, which contradicts the non-negativity of $u$. Hence $\lim _{\tau \searrow 0} x_{*}(\tau)=c$. This completes the proof of the lemma.

\subsection{THE LIMIT OF THE APPROXIMATION PROBLEM}

Consider the family $\left\{x^{\varepsilon}\right\}_{0<\varepsilon<\varepsilon_{0}}$. It is a bounded family of decreasing functions. We can select a sequence $\left\{\varepsilon_{j}\right\}$ and find a decreasing function $x$ such that as $j \rightarrow \infty, \varepsilon_{j} \searrow 0$ and $x^{\varepsilon_{j}}(\tau) \longrightarrow x(\tau)$ for every $\tau \geqslant 0$. Clearly $x(0)=c$. In view of Lemma 5.3.1, $x_{*}(\tau) \leqslant x(\tau) \leqslant x^{*}(\tau)$. Hence, $x(\tau)<c \quad \forall \tau>0$ and $\lim _{\tau \searrow 0} x(\tau)=c=x(0)$.

Fix any small $\delta>0$. When $\varepsilon$ is sufficiently small, $x^{\varepsilon}(\delta)<x^{*}(\delta)+\left[c-x^{*}(\delta)\right] / 2$. It then follows from (5.6) that when $\tau \geqslant \delta$ and $0<\varepsilon \ll 1$,

$$
0 \leqslant-\frac{d x^{\varepsilon}(\tau)}{d \tau} \leqslant \frac{c \sigma^{2} K_{1}}{2\left[1-e^{-c \tau}\right]\left[c-x^{\varepsilon}\right]+4 \varepsilon} \leqslant \frac{c \sigma^{2} K_{1}}{\left[1-e^{-c \delta}\right]\left[c-x^{*}(\delta)\right]}=: K_{2}(\delta)
$$

Passing to the limit, we see that $x$ is Lipschitz continuous in $[\delta, \infty)$ with Lipschitz constant $K_{2}(\delta)$.

Next we define

$$
T^{*}=\sup \{\tau>0 \mid x(\tau)>0\} .
$$


Fix an arbitrary $T \in\left(\delta, T^{*}\right)$. Then $0<x(T) \leqslant x(\tau)$ for all $\tau \in[0, T]$. It then follows that $x^{\varepsilon_{j}}(T)>x(T) / 2$ for all $j$ sufficiently large. Consequently, by Lemma 5.2.1,

$$
0 \leqslant u_{r}^{\varepsilon_{j}}(r, \tau) \leqslant K_{1} x^{\varepsilon_{j}}(\tau)^{-1} \leqslant 2 K_{1} x(T)^{-1} \quad \forall r \geqslant x^{\varepsilon_{j}}(\tau), \tau \in[0, T], j \gg 1 .
$$

By a bootstrap argument, one can establish uniform (in $\varepsilon$ ) bounds for any higher order derivatives of $x^{\varepsilon}$ in $[2 \delta, T]$ and $u^{\varepsilon}$ in $\overline{Q_{\varepsilon}} \cap(0, \infty) \times[2 \delta, T]$. Hence, along a subsequence, $\left\{u^{\varepsilon_{j}}\left(r+\left[x^{\varepsilon_{j}}(\tau)-\right.\right.\right.$ $x(\tau)], \tau)\}$ approaches a limit $u$ and $(u, x)$ satisfies

$$
\begin{gathered}
x \in C([0, \infty)) \cap C^{\infty}\left(\left(0, T^{*}\right)\right), \quad u \in C^{\infty}\left(\left\{(r, \tau) \mid \tau \in\left(0, T^{*}\right), r \geqslant x(\tau)\right\}\right), \\
u_{\tau}-\mathcal{L} u=r-c \quad \forall r>x(\tau), 0<\tau<T^{*} . \\
u(x(\tau), \tau)=0, \quad \frac{d x}{d \tau}=\frac{c \sigma^{2} x u_{r}(x, \tau)}{2\left(1-e^{-c \tau}\right)(c-x)} \quad \forall \tau \in\left(0, T^{*}\right), \\
u(r, 0)=0 \quad \forall r \geqslant c, \quad 0<x(\tau)<c \quad \forall \tau \in\left(0, T^{*}\right) .
\end{gathered}
$$

Since we already know that $v=u_{\tau} \geqslant 0$ and $x^{\prime} \leqslant 0$, by a similar argument analogous to the previous subsection, we can show that $x^{\prime}<0$ in $\left(0, T^{*}\right)$.

\subsection{EXISTENCE OF A CLASSICAL SOLUTION OF (5.4).}

Note that if $T^{*}=\infty$, the limit $(u, x)$ obtained in the previous subsection is a classical solution of (5.4). Here, we consider the case $T^{*}<\infty$.

Since $u_{\tau}^{\varepsilon}>0>x_{\tau}^{\varepsilon \prime}$, along a subsequence of $\left\{\varepsilon_{j}\right\}, u^{\varepsilon_{j}}$ approaches a function $\underline{u}$ locally uniformly in $(0, \infty) \times\left[T^{*}, \infty\right)$. In addition,

$$
\underline{u}>0, \quad \underline{u}_{r}>0, \quad \underline{u}_{\tau}>0, \quad \underline{u}_{\tau}-\mathcal{L} \underline{u}+c-r=0 \quad \text { in }(0, \infty) \times\left[T^{*}, \infty\right) .
$$

We now define $u$ on $(0, \infty) \times\left[T^{*}, \infty\right)$ as the unique bounded solution of

$$
\begin{aligned}
& u_{\tau}-\mathcal{L} u=r-c \text { in }(0, \infty) \times\left(T^{*} \infty\right), \\
& \lim _{r \searrow 0} r^{b} u_{r}(r, \tau)=0 \forall \tau>T^{*}, \quad u\left(\cdot, T^{*}\right)=\underline{u}\left(\cdot, T^{*}\right) .
\end{aligned}
$$


Since $\underline{u}_{r} \geqslant 0$ and $0 \leqslant \underline{u} \leqslant 1$, the function $r^{b} \underline{u}_{r}$ is integrable. For $\nu=2 \kappa / \sigma^{2}$ and any non-negative smooth function $\zeta$ compactly supported on $[0, \infty) \times\left(T^{*}, \infty\right)$, one can integrate the equation $r^{b-1} e^{-\nu r}\left[\underline{u}_{\tau}-\mathcal{L} \underline{u}+c-r\right] \zeta=0$ over $[\delta, \infty) \times\left[T^{*}, \infty\right)$ and send $\delta \searrow 0$ to obtain

$$
\lim _{\delta \searrow 0} \frac{\sigma^{2}}{2} \int_{T^{*}}^{\infty} \delta^{b} \underline{u}_{r}(\delta, \tau) \zeta(0, \tau) d \tau=\int_{T^{*}}^{\infty} \int_{0}^{\infty} e^{-\nu r}\left[r^{b-1} \underline{u} \zeta_{\tau}-\frac{\sigma^{2}}{2} r^{b} \underline{u}_{r} \zeta_{r}+r^{b} \underline{u} \zeta\right] d r d \tau<\infty
$$

Hence,

$$
h(d \tau):=\lim _{r \searrow 0} r^{b} \underline{u}_{r}(r, \cdot) d \tau
$$

is a non-negative measure on $\left[T^{*}, \infty\right)$. Then one can use Green's representation (Lemma A.3.1) to conclude that $\underline{u} \leqslant u$ on $(0, \infty) \times\left[T^{*}, \infty\right)$. This implies that $u>0$ on $(0, \infty) \times\left[T^{*}, \infty\right)$. Using $u_{\tau}\left(r, T^{*}\right)=\mathcal{L} \underline{u}\left(r, T^{*}\right)+c-r^{*}=\underline{u}_{\tau}\left(r, T^{*}\right)>0$, we can show that $u_{\tau}>0$ in $(0, \infty) \times\left[T^{*}, \infty\right)$. Using $\underline{u}_{r}\left(\cdot, T^{*}\right)>0$ we also derive that $u_{r}>0$ in $(0, \infty) \times\left[T^{*}, \infty\right)$.

Thus, taking this $u$ as the extension of the function $u$ defined in the previous subsection, we obtain a solution of (5.4).

We summarize our analysis with the following:

Theorem 3. The free boundary problem (5.4) admits a solution that has the following properties:

1. There exists a constant $T^{*} \in(0, \infty]$ such that $x \in C([0, \infty)) \cap C^{\infty}\left(\left(0, T^{*}\right)\right)$,

$$
x=0 \text { in }\left[T^{*}, \infty\right), \quad x>0>x^{\prime} \geqslant \frac{c \sigma^{2} K_{1}}{2\left(1-e^{-c \tau}\right)(x-c)} \text { in }\left(0, T^{*}\right) .
$$

2. Set $Q=\{(r, \tau) \mid \tau>0, r>x(\tau)\}$ and $\Gamma=\left\{(x(\tau), \tau) \mid \tau \in\left(0, T^{*}\right)\right\}$. Then $u \in C(\bar{Q} \backslash$ $\left.\left\{\left(0, T^{*}\right)\right\}\right) \cap C^{\infty}(Q \cup \Gamma), u>0, u_{\tau}>0, u_{r}>0$ in $Q$, and $u_{r} \leqslant(1-u) \max \left\{b, c \kappa^{-1}\right\}$ when $\tau<T^{*}$. 


\subsection{THE NON-UNIQUENESS ISSUE}

Without the no-flux boundary condition, clearly, $\underline{u}$ is a solution of (5.4). However, when $b \in(0,1)$ and $T^{*}<\infty$, we can show that $\underline{u} \neq u$ as follows.

(i) By the analysis of the infinite horizon problem, we know that there exists a positive number $c_{*}$ such that if $c>c_{*}$, then there exist $R_{*} \in(0, c)$ and a smooth bounded function $V_{*}(r)$ defined on $\left[R_{*}, \infty\right)$ such that $V_{*}\left(R_{*}\right)=0, V_{*}^{\prime}\left(R_{*}\right)=0, V_{*}^{\prime}<0=\mathcal{L} V_{*}+c$ on $\left(R_{*}, \infty\right)$ and $V_{*}(\infty):=$ $\lim _{r \rightarrow \infty} V_{*}(r)=0$. Set $U_{*}:=1-V_{*}$. Then by comparison, one can show that $u^{\varepsilon}(r, \tau) \leqslant U_{*}(r)$ and $x^{\varepsilon}(\tau)>R_{*}$ for every $\varepsilon \in\left(0, \varepsilon_{0}\right), \tau \in[0, \infty)$ and $r \in\left[x^{\varepsilon}(\tau), \infty\right)$. Consequently, $x(\tau)>R_{*}$ for all $\tau>0$ and $T^{*}=\infty$. Furthermore, by monotonicity $u_{\tau}>0>x^{\prime}$ and uniqueness of the infinite horizon problem, we have $\lim _{\tau \rightarrow \infty}(x(\tau), u(\cdot, \tau))=\left(R_{*}, U_{*}\right)$.

(ii) When $c=c^{*}$, there exists a function $V_{* *}$ define on $[0, \infty)$ such that $V_{* *}(0)=1, V_{* *}^{\prime}(0)=$ $-c /(\kappa \theta)<0, V_{* *}^{\prime}<0=\mathcal{L} V_{* *}+c^{*}$ on $(0, \infty)$ and $V_{* *}(\infty)=0$.

Now assume that $b \in(0,1)$. Then by Lemma 3.1.1, there exists a function $G_{1} \in C([0, \infty)) \cap$ $C^{\infty}((0, \infty))$ such that $\mathcal{L}\left[G_{1}\right]=0$ on $(0, \infty), G_{1}^{\prime}<0<G_{1}$ on $(0, \infty)$ and $G_{1}(\infty)=0$.

When $c \in\left(0, c^{*}\right]$, consider the function

$$
U:=\frac{c}{c^{*}}\left[1-V_{* *}\right]+\left(1-\frac{c}{c^{*}}\right)\left(1-\frac{G_{1}(r)}{G_{1}(0)}\right) .
$$

Then one calculate, $U(0)=0, U^{\prime}>0$ on $(0, \infty)$, and

$$
\mathcal{L} U+r-c=\frac{c}{c^{*}}\left(c^{*}-r\right)+\left(1-\frac{c}{c^{*}}\right) r+r-c=0 \text { on }(0, \infty) .
$$

Then by comparison, $U(r)>u^{\varepsilon}(r, \tau)$ for any $\varepsilon \in\left(0, \varepsilon_{0}\right), \tau>0$ and $r \geqslant x^{\varepsilon}(\tau)$. Consequently, $\underline{u}(r, \tau) \leqslant U(r)$ for every $r \in[0, \infty)$ and $\tau \in\left[T^{*}, \infty\right)$. This implies that $\underline{u}$ is continuous on $[0, \infty) \times\left[T^{*}, \infty\right)$ and $\underline{u}=0$ on $\{0\} \times\left[T^{*}, \infty\right)$. On the other hand, as $\tau \rightarrow \infty, u(\cdot, \tau)$ approaches the solution of the infinite horizon problem given by $U_{*}=\left(c / c^{*}\right)\left[1-V_{* *}\right]+\left[1-c / c^{*}\right]$. Thus, $\lim _{\tau \rightarrow \infty} u(0, \tau)=1-c / c^{*}$ and $\underline{u} \neq u$ when $c \in\left(0, c_{*}\right)$. Hence, if no-flux boundary condition is

removed, there are infinitely many solutions; for example, for any $\theta \in(0,1), \theta u+(1-\theta) \underline{u}$ is a solution.

The super-solution $U$ constructed in (5.8) and the conclusion $\underline{u} \leqslant U$ immediately give us the following:

Corollary 5.6.1. When $b \in(0,1)$ and $T^{*}<\infty$, $u$ is continuous at $\left(0, T^{*}\right)$ and $u\left(0, T^{*}\right)=0$. 


\subsection{EQUIVALENCY OF THE FREE BOUNDARY PROBLEM (5.4) AND THE VARIATIONAL INEQUALITY (2.1)}

Without knowing the regularity of free boundary one cannot construct a classical solution of the free boundary (5.4) from a (viscosity) solution of (2.1). Here we show that the reverse is true; namely, we can construct a solution $W$ of the variational inequality (2.1) from a solution $(u, x)$ of the free boundary problem (5.4). Once this is done, we conclude from the uniqueness of solution of (2.1) that the solution of (5.4) is also unique. In addition, by existence of smooth classical solution of (5.4), we know that the free boundary of the solution of (2.1) is smooth.

Let $(u, x)$ be a smooth solution of (5.4).

(i) We recall that the free boundary problem (5.4) is formally derived for the function $u=$ $W_{\tau}+c W=e^{-c \tau}\left(e^{c \tau} W\right)_{\tau}$. Hence, it is natural to recover $W$ from $u$ by setting

$$
W(r, \tau):=\int_{0}^{\tau} e^{c(t-\tau)} u(r, t) d t \quad \forall r>0, t \geqslant 0 .
$$

Here $u$ is extended to $(0, \infty) \times[0, \infty)$ by $u(r, \tau)=0$ for all $r \in(0, x(\tau))$ and $\tau \geqslant 0$. Since $x$ is a decreasing function, we have $W(r, \tau)=0$ when $r \in(0, x(\tau))$ and $W>0$ when $r>x(\tau)$. In addition, as $u$ is continuous and $u_{r}$ is locally bounded,

$$
W_{\tau}(r, \tau)=u(r, \tau)-c W(r, \tau), \quad W_{r}=\int_{0}^{\tau} e^{c(t-\tau)} u_{r}(r, t) d t=\int_{S(r)}^{\tau} u_{r}(r, t) d t
$$

where $S(r)=0$ if $r \geqslant c, x(S(r))=r$ if $c>r \geqslant x(\tau)$ and $S(r)=\tau$, if $r<x(\tau)$. Thus, $W_{r}, W_{\tau}$ are continuous in $(0, \infty) \times(0, \infty)$. In particular, $W_{r}(x(\tau), \tau)=0$ when $x(\tau)>0$.

(ii) (a) First consider $(r, \tau) \in \Sigma_{1}=\{(r, \tau) \mid \tau>0,0<r<x(\tau)\}$. Since $x$ is continuous, $W \equiv 0$ in an open neighborhood of $(r, \tau)$. Consequently, $W_{\tau}(r, \tau)-\mathcal{L} W(r, \tau)+P(r, \tau)=$ $P(r, \tau)>0$.

(b) Next consider $(r, \tau) \in Q_{1}=[c, \infty) \times(0, \infty)$. We have $W(r, \tau)>0$ and

$$
\begin{aligned}
\mathcal{L} W(r, \tau) & =\int_{0}^{\tau} e^{c(t-\tau)} \mathcal{L} u(r, t) d t=\int_{0}^{\tau} e^{c(t-\tau)}\left[u_{t}(r, t)+c-r\right] d t \\
& =u(r, \tau)-c \int_{0}^{t} e^{c(t-\tau)} u(r, t) d t+(c-r) M(\tau)=W_{\tau}+(c-r) M(\tau) .
\end{aligned}
$$

Thus, $W_{\tau}-\mathcal{L} W+P=0<W$ in $[c, \infty) \times(0, \infty)$. 
(c) Now consider $(r, \tau) \in Q_{2}:=\{(r, \tau) \mid \tau>0, x(\tau)<r<c\}$. Then $S^{\prime}(r)=1 / x^{\prime}(S(r))$ so

$$
\begin{aligned}
W_{r r}(r, \tau) & =\int_{S(r)}^{\tau} e^{c(t-\tau)} u_{r r}(r, t) d t-S^{\prime}(r) e^{c(S(r)-\tau)} u_{r}(r, S(r)) \\
& =\int_{S(r)}^{\tau} e^{c(t-\tau)} u_{r r}(r, t) d t+e^{c(S(r)-\tau)} \frac{2\left(1-e^{-c S(r)}\right)(c-r)}{c \sigma^{2} r} .
\end{aligned}
$$

Hence,

$$
\begin{aligned}
\mathcal{L} W & =\int_{S(r)}^{\tau} e^{c(t-\tau)} \mathcal{L} u(r, t) d t+\frac{e^{c(S(r)-\tau)}\left(1-e^{-c S(r)}\right)(c-r)}{c} \\
& =\int_{S(r)}^{\tau}\left[u_{t}(r, t)+c-r\right] e^{c(t-\tau)} d t+\frac{e^{c(S(r)-\tau)}\left(1-e^{-c S(r)}\right)(c-r)}{c} \\
& =u(r, \tau)-c \int_{S(r)}^{t} e^{c(t-\tau)} u(r, t) d t+\frac{(c-r)}{c}\left\{1-e^{-c \tau)}\right\} \\
& =W_{\tau}(r, \tau)+(c-r) M(\tau) .
\end{aligned}
$$

Thus, we have $W_{\tau}-\mathcal{L} W+P=0<W$ in $Q_{1} \cup Q_{2}=\{(r, \tau) \mid \tau>0, r>x(\tau)\}=Q$.

(d) Finally consider the free boundary $\Gamma=\{(r, \tau) \mid \tau>0, r=x(\tau)>0\}$. Suppose $\left(r_{0}, \tau_{0}\right) \in$ $\Gamma, \psi$ is smooth and $\psi \leqslant W$ in a neighborhood of $\left(r_{0}, \tau_{0}\right)$ and $\psi\left(r_{0}, \tau_{0}\right)=0=W\left(r_{0}, \tau_{0}\right)$. Then since $W\left(r_{0}, t\right)=0$ for $t \in\left(0, \tau_{0}\right]$, we have $\psi_{\tau}\left(r_{0}, \tau_{0}\right) \geqslant 0$. Also, $\psi_{r}\left(r_{0}, \tau_{0}\right)=W_{r}\left(r_{0}, \tau_{0}\right)=0$. As $\psi\left(r, \tau_{0}\right) \leqslant W\left(r, \tau_{0}\right)=0$ for $r \in\left(0, r_{0}\right]$, we see that $\psi_{r r}\left(r_{0}, \tau_{0}\right) \leqslant 0$. Hence, we obtain

$$
\psi_{\tau}\left(r_{0}, \tau_{0}\right)-\mathcal{L} \psi\left(r_{0}, \tau_{0}\right)+P\left(r_{0}, \tau_{0}\right) \geqslant P\left(r_{0}, \tau_{0}\right)>0
$$

This means that $W_{\tau}-\mathcal{L} W+P>0$ on $\Gamma$ in the viscosity sense.

In summary, we obtain

$$
\min \left\{W_{\tau}-\mathcal{L} W+P, W\right\}=0 \quad \text { in }(0, \infty) \times(0, \infty)
$$

(iii) It remains to verify the no-flux condition, which is needed only when $b \in(0,1)$. In this case we know that $u$ is continuous at $\left(0, T^{*}\right)$.

When $\tau<T^{*}$, we have $W(r, \tau)=0$ for $r \in(0, x(\tau))$ so $\lim _{r \searrow 0} r^{b} W_{r}(r, \tau)=0$. Thus, $W$ is a the unique solution of $(2.1)$ on $[0, \infty) \times\left[0, T^{*}\right]$.

When $\tau \geqslant T^{*}$,

$$
\lim _{r \searrow 0} r^{b} W_{r}(r, \tau)=\lim _{r \searrow 0} r^{b} W_{r}\left(r, T^{*}\right) e^{-c\left(\tau-T^{*}\right)}+\int_{T^{*}}^{\tau} e^{c(t-\tau)} \lim _{r \searrow 0} r^{b} u_{r}(r, t) d t=0 .
$$


Here we have used the fact that in $[0, \infty) \times\left[0, T^{*}\right]$, the solution of $(2.1)$ is unique and the solution is Lipschitz continuous, so $r^{b} W_{r}\left(r, T^{*}\right) \rightarrow 0$ as $r \searrow 0$. Thus, $W$ is a solution of $(2.1)$ on $[0, \infty) \times$ $[0, \infty)$. We summarize our calculation as follows:

Lemma 5.7.1. Let $(u, x)$ be the smooth solution of (5.4). Then $W$ defined in (5.9) is a solution of (2.1) with a smooth free boundary.

\subsection{THE FUNCTION $W_{\tau}$}

In Theorem 2, we have shown that $-c M \leqslant W_{\tau} \leqslant M^{\prime}$. Here we establish additional properties of $W_{\tau}$. From $W_{\tau}=u-c W$ we see that $W \in C\left(\bar{Q} \backslash\left\{\left(0, T^{*}\right)\right\}\right)$ and $W_{\tau}=0$ on $\Gamma$. In addition, from $\left(e^{c \tau} W_{\tau}\right)_{\tau}=e^{c \tau} u_{\tau} \geqslant 0$, the condition $W_{\tau}(r, 0)=0$ for $r>0, W_{\tau}(x(\tau), \tau)=0$ for $\tau \in\left(0, T^{*}\right)$, and $x^{\prime}<0$ on $\left(0, T^{*}\right)$, we derive that when $W_{\tau}(r, \tau)=\int_{S(r)}^{\tau} e^{c(t-\tau)} u_{\tau}(c, t) d t>0$ for $\tau>0$ and $r>x(\tau)$. Hence, we have the following

Theorem 4. Let $v:=W_{\tau}=u-c W$. Then $\left(v, x, T^{*}\right)$ is a classical solution of the following free boundary problem: with $Q:=\{(r, \tau) \mid \tau>0, r>x(\tau)\}$ and $\Gamma=\left\{(r(\tau), \tau) \mid 0<\tau<T^{*}\right\}$, $x \in C([0, \infty)) \cap C^{\infty}\left(\left(0, T^{*}\right)\right), v \in C^{\infty}(\Gamma \cup Q) \cap C\left(\bar{Q} \backslash\left\{\left(0, T^{*}\right)\right\}\right)$, and

$$
\begin{cases}v_{\tau}-\mathcal{L} v+P_{\tau}=0<v & \text { in } Q, \\ v(x(\tau), \tau)=0<x(\tau)<c & \text { if } \tau \in\left(0, T^{*}\right), \\ 0<-\frac{d x}{d \tau}=\frac{c \sigma^{2} x v_{r}(x, \tau)}{2\left[1-e^{-c \tau}\right][c-x]} & \text { if } \tau \in\left(0, T^{*}\right), \\ \lim _{r \searrow 0} r^{b} v_{r}(r, \tau)=0=x(\tau) & \text { if } \tau \in\left(T^{*}, \infty\right), \\ x(0)=c, v(\cdot, 0)=0 . & \end{cases}
$$

Consequently, as $\tau \rightarrow \infty, W(\cdot, \tau) \nearrow W^{*}:=1-V^{*}, u=W_{\tau}+c W \nearrow c W^{*}$, and $x(\tau) \searrow R^{*}$ where $\left(R^{*}, V^{*}\right)$ is the solution of the infinite horizon problem.

This free boundary problem is almost the same as (5.4) except $c-r$ is replaced by $P_{\tau}$. In the study of American put option, the function $v$ played an important role in both mathematical analysis and in numerical evaluation, see $[10,9,11,12,13]$. 


\subsection{CALIBRATING THE COX-INGERSOLL-ROSS MODEL}

As we discussed earlier, the short rate is the annualized interest rate for an infinitesimally short period of time. Short-rate models, such as the Vasicek or CIR models, can be calibrated to historical data for a realized interest rate. However, in our derivation of the variational inequality, an affine term structure assumption was necessary in order to develop the hedging strategy. That is, we assumed ATSM and by the fundamental theorem of asset pricing of Heath, Jarrow and Morton the existence of a short rate followed. We then specified the constants of Proposition 2.2.2 that reconcile the ATSM with the CIR model.

In this chapter, we implement a variety of statistical estimation methods to determine reasonable values of the parameters $\theta, \kappa$ and $\sigma$. The methods we consider reflect both the time-series aspect of the CIR model and the term structure aspect of how the CIR model relates to ATSM.

\subsection{STATISTICAL PROPERTIES OF THE CIR MODEL}

An essentially beneficial feature of the CIR model is both its closed form transition density function and the closed representation of bond prices. Both are required for our statistical analysis.

Recall the CIR assumes the short rate $r_{t}$ is governed by the stochastic differential equation

$$
d r_{t}=\kappa\left(\theta-r_{t}\right) d t+\sigma \sqrt{r_{t}} d W_{t}
$$

where $\kappa, \theta$ and $\sigma$ are positive constants.

The transition density function of the short rate at time $s$, conditional on its value at time $t$ can be expressed as

$$
p\left(r_{s} ; r_{t}\right)=c e^{-u-v}\left(\frac{v}{u}\right)^{(b-1) / 2} I_{b-1}(2 \sqrt{u v}) \quad \text { for } s \geqslant t
$$


with $c=\frac{2 \kappa}{\sigma^{2}\left(1-e^{-\kappa(s-t)}\right)}, u=c r_{t} e^{-\kappa(s-t)}, v=c r_{s}$ and $b=\frac{2 \kappa \theta}{\sigma^{2}}$. The modified Bessel function of the first kind of order $q$ is defined as

$$
I_{q}(z)=\sum_{j=0}^{\infty} \frac{1}{j ! \Gamma(q+j+1)}\left(\frac{z}{2}\right)^{2 j+q}
$$

with $\Gamma(\cdot)$ denoting the gamma function.

In the appendix we derive the Laplace transform of the conditional distribution of the short rate in the CIR model. From this we see that the CIR model can be represented as a non-central chi-square distribution with $2 b$ degrees of freedom and non-centrality parameter $2 u$, i.e.,

$$
2 c r_{s} \mid r_{t} \sim \chi_{2 b, 2 u}^{2} \quad \text { for } s \geqslant t
$$

Using properties of the Laplace transform or Itô's Lemma, we can derive the conditional mean and conditional variance

$$
\begin{aligned}
E\left(r_{s} \mid r_{t}\right) & =r_{t} e^{-\kappa(s-t)}+\theta\left(1-e^{-\kappa(t-s)}\right) \\
\operatorname{Var}\left(r_{s} \mid r_{t}\right) & =r_{t}\left(\frac{\sigma^{2}}{2 \kappa}\right)\left(e^{\kappa(s-t}-e^{-2 \kappa(s-t)}\right)+\frac{\sigma^{2} \theta}{2 \kappa}\left(1-e^{-\kappa(s-t)}\right)^{2}
\end{aligned}
$$

Using the conditional mean, we can also derive the covariance for $s \geqslant t$

$$
\operatorname{cov}\left(r_{s}, r_{t}\right)=\frac{\sigma^{2} r_{t}}{\kappa}\left(e^{-\kappa s}-e^{-\kappa(s+t)}\right)+\frac{\sigma^{2} \theta}{2 \kappa}\left(e^{-\kappa(s-t)}-2 e^{\kappa s}+e^{-\kappa(s+t)}\right)
$$

An essential feature of the CIR model is the fact that one can explicitly obtain bond prices as affine functions of the short-rate $r_{t}$. Rewriting the expression given in Corollary A.3.3, the return at time $t$ on the zero-coupon bond maturing at $T>t$ can be given to be

$$
-\log Z(r, \tau)=A(\tau)+r B(\tau)
$$

where $r$ is taken to be the short-rate $r_{t}, \tau:=T-t$ and $A(\cdot)$ and $B(\cdot)$ are given by

$$
A(\tau)=-b \log \left(\frac{\gamma \exp \left(\frac{\kappa \tau}{2}\right)}{\gamma \cosh \left(\frac{\gamma \tau}{2}\right)+\kappa \sinh \left(\frac{\gamma \tau}{2}\right)}\right), \quad B(\tau)=\frac{\sinh \left(\frac{\gamma \tau}{2}\right)}{\gamma \cosh \left(\frac{\gamma \tau}{2}\right)+\kappa \sinh \left(\frac{\gamma \tau}{2}\right)}
$$

where $b=\frac{2 \kappa \theta}{\sigma^{2}}$ and $\gamma=\sqrt{\kappa^{2}+2 \sigma^{2}}$ are the constants given in (2.2). 
We consider two varieties of parameter estimation in the attempt to take advantage of both the CIR model's distribution properties of $r_{t}$ as given by (6.1) and the financial applications as given by (6.6).

The first method we consider is a time series estimation using (6.1). In this case, maturity time is fixed and the parameters are estimated while considering the evolution of the interest rate over the different time periods of the dataset.

Our second method, using (6.6) is a cross-section estimation method using the entire term structure. In this case, we perform an estimation of the parameters while considering different maturities at fixed moments in time.

Naturally, both methods carry merits and drawbacks. For the time-series method, we have a closed-form expression for the transition density, however, we must choose a bond maturity whose yield to serve as proxy for the short rate. In the cross-section method, we again have closedform expression (for the yield curve), however, using data sets encompassing a variety of yield curve types (e.g., inverted, normal, etc.) can lead to dubious parameter estimates. In theory, the differences between time series and cross-sectional estimates should be small if one-factor models of the term structure were accurate over large time frames.

\subsection{U.S. TREASURY DATA}

In practical and theoretical finance, Treasury bonds issued by the United States are considered risk-free. We use the term structure provided by the U.S. Treasury for our calibration purposes. The Treasury's yield curve is derived using a quasi-cubic Hermite spline function. The inputs are the "close of business" bid yields for the on-the-run securities.

Specifically, the Treasury uses the most recently auctioned 4-, 13-, 26-, and 52-week bills, plus the most recently auctioned 2-, 3-, 5-, 7-, and 10- year notes and the most recently auctioned 30year bond, plus the composite rate in the 20-year maturity range. The rates obtained, are known as Constant Maturity Treasury (CMT) rates.

At the writing of this thesis, historical data sets for extended date ranges in addition for further technical details regarding the Treasury's numerical methods mentioned above can be found at the 
following site:

http://www.treasury.gov/resource-center/data-chart-center/interest-rates/

The Treasury provides CMT rates for 1-, 3-, and 6- month bills and 1-, 2-, 3-, 5-, 7-, 10-, 20-, and 30- year bonds. We collected data for the full term structure for the date range January 1, 2009 through December 31, 2011, given us 729 days of data for 11 different maturities.

We denote by the set $\left\{R_{i}^{j}\right\}$ the annualized CMT rate at trading day $t_{i}$, where $i=1, \ldots, 729$ given the maturity $j$, for $j \in\left\{\frac{1}{12}, \frac{3}{12}, \frac{6}{12}, 1,2,3,4,5,7,10,20,30\right\}$.

Table 1: US Treasury fixed-term bond yields, from Jan. 2, 2009 to Dec. 31, 2011.

\begin{tabular}{|c|c|c|c|c|c|c|c|c|c|c|c|}
\hline Date & $1 \mathrm{mo}$ & 3 mo & $6 \mathrm{mo}$ & $1 \mathrm{yr}$ & $2 \mathrm{yr}$ & $3 \mathrm{yr}$ & $5 \mathrm{yr}$ & $7 \mathrm{yr}$ & $10 \mathrm{yr}$ & $20 \mathrm{yr}$ & $30 \mathrm{yr}$ \\
\hline 3 Jan. 09 & 0.04 & 0.08 & 0.28 & $\overline{0.40}$ & 0.88 & 1.14 & 1.72 & 2.07 & 2.46 & 3.22 & 2.83 \\
\hline 1 Jul. 09 & 0.17 & 0.19 & 0.35 & 0.56 & 1.11 & 1.64 & 2.54 & 3.19 & 3.53 & 4.30 & 4.32 \\
\hline 1 Jan. 10 & 0.04 & 0.06 & 0.20 & 0.47 & 1.14 & 1.70 & 2.69 & 3.39 & 3.85 & 4.58 & 4.63 \\
\hline 1 Jul. 10 & 0.17 & 0.18 & 0.22 & 0.32 & 0.61 & 1.00 & 1.79 & 2.42 & 2.97 & 3.74 & 3.91 \\
\hline 1 Jan. 11 & 0.07 & 0.12 & 0.19 & 0.29 & 0.61 & 1.02 & 2.01 & 2.71 & 3.30 & 4.13 & 4.34 \\
\hline 1 Jul. 11 & 0.01 & 0.03 & 0.10 & 0.19 & 0.45 & 0.81 & 1.76 & 2.50 & 3.18 & 4.09 & 4.38 \\
\hline 31 Dec. 11 & 0.01 & 0.02 & 0.06 & 0.12 & 0.25 & 0.36 & 0.83 & 1.35 & 1.89 & 2.57 & 2.89 \\
\hline mean & 0.083 & 0.11 & 0.19 & 0.32 & 0.70 & 1.09 & 1.88 & 2.53 & 3.09 & 3.92 & 4.08 \\
\hline std. dev. & 0.059 & 0.07 & 0.10 & 0.145 & 0.28 & 0.42 & 0.54 & 0.58 & 0.55 & 0.53 & 0.51 \\
\hline
\end{tabular}

The choice of date range was restricted by two fundamental considerations, the first consideration is that the full range of maturities currently traded was not available prior to February 2006. The second was that from 2006 to 2009, the yield curve displayed highly erratic behavior over time, taking on a variety of shapes (inverted, flat and humped). Calibrating a one-factor model over such a variety of yield curve shapes can lead to poor, often erroneous estimation results.

The collection of yield curves over time is called a yield surface. In the figure below, we plot the yield surface over the time-frame of our calibration. We note that for the date range, the typical yield curve shape is the normal type, i.e., increasing as a function of maturity and concave. 
Figure 2: The U.S. Treasury yield surface from January 1, 2009 to December 31, 2011

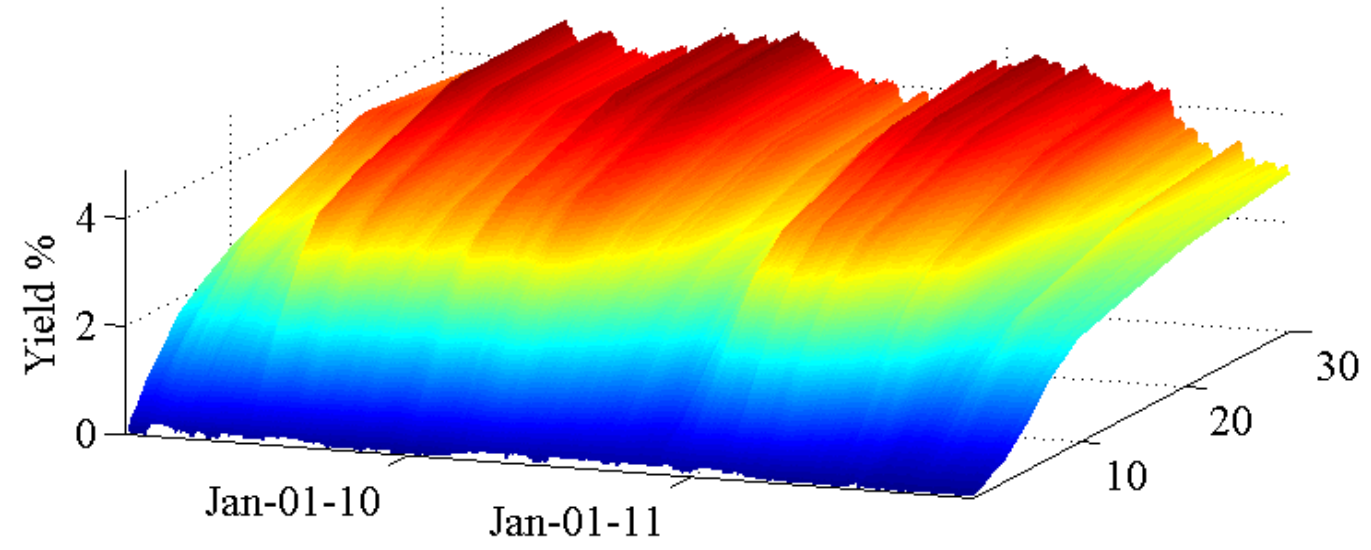

\subsection{TIME SERIES ANALYSIS}

We use the maximum likelihood method as an estimation technique to estimate the parameters in the vector $\kappa, \theta$, and $\sigma$ is given in (6.1). To begin, we must choose a time series to approximate the short rate process, i.e., we choose a maturity and use the yields of that maturity to approximate $r_{t}$.

Let $r_{i}:=r_{t_{i}}$ where $t_{i}=i \Delta t$ and assume $\Delta t:=t_{i+1}-t_{i}$ constant with $\left[t_{i}, t_{i}+\Delta t\right)$ are not overlapping.

The maximum log-likelihood function is defined as

$$
\begin{aligned}
\Psi(\kappa, \theta, \sigma) & :=\ln \prod_{i=0}^{n} p\left(r_{i+1} ; r_{i}\right) \\
& =\sum_{i=0}^{n} \ln \left\{p\left(r_{i+1} ; r_{i}\right)\right\} \\
& =\sum_{i=0}^{n} \ln \left\{e^{-u_{i}-v_{i}}\left(\frac{v_{i}}{u_{i}}\right)^{(b-1) / 2} I_{\frac{b-1}{2}}\left(2 \sqrt{u_{i} v_{i}}\right)\right\} .
\end{aligned}
$$

with $c=\frac{2 \kappa}{\sigma^{2}\left(1-e^{-\kappa \Delta t}\right)}, u_{i}=c r_{i} e^{-\kappa \Delta t}$ and $v_{i}=c r_{i+1}$. Simplifying the above equation, the maximum log-likelihood function becomes

$$
\Psi(\kappa, \theta, \sigma)=n \ln c+\sum_{i=1}^{n}\left\{-u_{i}-v_{i}+\frac{1}{2}(b-1) \ln \left(\frac{v_{i}}{u_{i}}\right)+\ln I_{\frac{b-1}{2}}\left(2 \sqrt{u_{i} v_{i}}\right)\right\}
$$


The maximum likelihood estimates are found by maximizing $\Psi$ over its parameter space, i.e.,

$$
(\sigma, \kappa, \theta)=\underset{\sigma, \kappa, \theta}{\operatorname{argmax}} \Psi(\kappa, \theta, \sigma)
$$

The maximum log-likelihood function $\Psi$ is nonlinear in the variables $u$ and $v$ rendering the optimization of $\Psi$ a nontrivial problem. The problem is further exacerbated by the presence of the modified Bessel function of the first kind. Therefore we investigate suitable initial estimates of the parameters $\kappa, \theta$ and $\sigma$. For the remainder of this chapter we describe various methods for obtaining initial estimates and conclude with a summary of our analysis.

Initial estimates of the parameters are crucial for convergence in the numerical optimization method. We consider initial estimates obtained by s covariance-equivalent and moment matching difference equation representation of the CIR model.

We begin with stochastic differential equation for the CIR model (6.1) which we write as

$$
d\left(e^{-\kappa t}\left(r_{t}-\theta\right)\right)=e^{-\kappa t} \sigma \sqrt{r_{t}} d W_{t}
$$

Make the change of variables

$$
x_{t}:=r_{t}-\theta, \quad x_{0}:=r_{0}-\theta .
$$

Then (6.9) becomes

$$
\begin{aligned}
d\left(e^{-\kappa t} x_{t}\right) & =e^{-\kappa t} \sigma \sqrt{x_{t}+\theta} d W_{t} \\
d x_{t} & =-\kappa x_{t} d t+\sigma \sqrt{x_{t}+\theta} d W_{t} .
\end{aligned}
$$

with the initial condition $x_{0}=\left(r_{0}-\theta\right)$.

Notice the process $x_{t}$ is centered around $\theta$. This does not introduce any additional difficulty as the expected values between $r_{t}$ and $x_{t}$ only differ by $\theta$ and the covariance functions are the same, which we demonstrate below.

We can solve (6.11) for $x_{t}$ to obtain

$$
x_{t}=e^{-\kappa t} x_{0}+\sigma e^{-\kappa t} \int_{0}^{t} e^{\kappa u} \sqrt{x_{u}+\theta} d W_{u} .
$$


The expected value immediately follows

$$
E\left(x_{t}\right)=e^{-\kappa t} x_{0}
$$

We calculate the covariance between $x_{t}$ and $x_{s}$ by definition

$$
\begin{aligned}
\operatorname{cov}\left(x_{t}, x_{s}\right) & =E\left[\left(x_{t}-E\left(x_{t}\right)\right)\left(x_{s}-E\left(x_{s}\right)\right)\right] \\
& =E\left[\left(\sigma e^{-\kappa t} \int_{0}^{t} e^{\kappa u} \sqrt{x_{u}+\theta} d W_{u}\right)\left(\sigma e^{-\kappa t} \int_{0}^{t} e^{\kappa v} \sqrt{x_{v}+\theta} d W_{v}\right)\right] \\
& =\sigma^{2} e^{-\kappa(t+s)} \int_{0}^{t} e^{2 \kappa v} E\left[x_{v}+\theta\right] d v \\
& =\sigma^{2} e^{-\kappa(s+t)} \int_{0}^{t} e^{2 \kappa v}\left(e^{-\kappa v} x_{0}+\theta\right) d v \\
& =\sigma^{2} x_{0} \frac{e^{-\kappa t}-e^{-\kappa(s+t)}}{\kappa}+\sigma^{2} \theta \frac{e^{-\kappa(t-s)}-e^{-\kappa(t+s)}}{2 \kappa}, \quad \text { for } s \leqslant t .
\end{aligned}
$$

The variance follows:

$$
\operatorname{Var}\left(x_{t}\right)=\operatorname{cov}\left(x_{t}, x_{t}\right)=-\frac{\sigma^{2}}{2 \kappa}\left(2 x_{0}+\theta\right) e^{-2 \kappa t}+\frac{\sigma^{2} x_{0}}{\kappa} e^{-\kappa t}+\frac{\sigma^{2} \theta}{2 \kappa} .
$$

The stationary variance is obtained by taking $t \nearrow \infty$

$$
\lim _{t \nearrow \infty} \operatorname{Var}\left(x_{t}\right)=\frac{\sigma^{2} \theta}{2 \kappa}
$$

which are the same as the conditional mean and variance (6.3) and covariance (6.5) from above.

Consider then the discrete model given by

$$
x_{t}=\phi x_{t-1}+S_{d}\left(\sqrt{\frac{2 \phi}{1+\phi} x_{t-1}+\theta}\right) e_{t}, \quad t=1,2,3, \ldots
$$

where $\phi$ and $S_{d}$ are positive constants and $e_{t}$ are independently identically distributed $N(0,1)$ random variables. 
Remark 6.3.1. There are other representations could be used instead of (6.13), namely

$$
x_{t}=\phi x_{t-1}+S_{d} \sqrt{x_{t-1}+\theta} e_{t}, \quad t=1,2,3, \ldots
$$

that will have the same first and second moments. However, it will not have an equivalent covariance. We derive (6.13) by setting

$$
x_{t}=\phi x_{t-1}+S_{e} \sqrt{\psi x_{t-1}+\theta} e_{t}
$$

derive the condition under which the discrete and continuous covariance functions will be equivalent. In doing so, we find $\psi=2 \phi /(1+\phi)$.

We can write (6.13) in a recursive way as

$$
x_{t}=\phi^{t} x_{0}+\sum_{i=0}^{t-1} \phi^{i} S_{d} \sqrt{\frac{2 \phi}{1+\phi} x_{t-i-1}+\theta} e_{t-i},, \quad t=1,2,3, \ldots
$$

The expected value is the same as above

$$
E\left(x_{t}\right)=\phi^{t} x_{0}
$$

Therefore, the parametric relation

$$
\phi=e^{-\kappa}
$$

must hold for the continuous and discrete processes to have the same expected value.

By definition, we calculate the covariance between $x_{t}$ and $x_{s}$ as follows:

$$
\begin{aligned}
\operatorname{cov}\left(x_{t}, x_{s}\right) & =E\left[\sum_{i=0}^{t-1} \phi^{i} S_{d} \sqrt{\frac{2 \phi}{1+\phi} x_{t-i-1}+\theta} e_{t-i} \sum_{j=0}^{s-1} \phi^{j} S_{d} \sqrt{\frac{2 \phi}{1+\phi} x_{s-j-1}+\theta e_{s-j}}\right] \\
& =\sum_{i=0}^{t-1} \sum_{j=0}^{s-1} \phi^{i+j} S_{d} E\left[\sqrt{\left.\frac{2 \phi}{1+\phi} x_{t-i-1}+\theta e_{t-i} \sqrt{\frac{2 \phi}{1+\phi} x_{s-j-1}+\theta e_{s-j}}\right]} .\right.
\end{aligned}
$$


Since the $e_{t}$ 's are identical and independently distributed $N(0,1)$ random variables, we can reduce the double summation to a single summation.

$$
\begin{aligned}
\operatorname{cov}\left(x_{t}, x_{s}\right) & =\sum_{j=0}^{s-1} \phi^{t-s+2 j} S_{d}^{2} E\left[\frac{2 \phi}{1+\phi} x_{s-j-1}+\theta\right] \\
& =\sum_{j=0}^{s-1} \phi^{t-s+2 j} S_{d}^{2}\left(\frac{2 \phi}{1+\phi} x_{0}+\theta\right) \\
& =\sum_{j=0}^{s-1} S_{d}^{2} \frac{2 \phi^{t+j}}{1+\phi} x_{0}+\sum_{j=0}^{s-1} \phi^{t-s+2 j} S_{d}^{2} \theta
\end{aligned}
$$

Expanding these partial geometric (in terms of $\phi$ ) sums, we get

$$
\operatorname{cov}\left(x_{t}, x_{s}\right)=2 \phi^{t} S_{d}^{2} x_{0} \frac{1-\phi^{s}}{1-\phi^{2}}+\phi^{t-s} S_{d}^{2} \theta \frac{1-\phi^{2 s}}{1-\phi^{2}}, \quad s \leqslant t
$$

The variance and stationary variance are then

$$
\begin{aligned}
\operatorname{Var}\left(x_{t}\right) & =2 \phi^{2} S_{d}^{2} x_{0} \frac{1-\phi^{2}}{1-\phi^{2}}+S_{d}^{2} \theta \frac{1-\phi^{2}}{1-\phi^{2}} \\
\lim _{t \nearrow \infty} & =\frac{S_{d}^{2} \theta}{1-\phi^{2}}
\end{aligned}
$$

Notice that while the stationary variance is the same for both difference equation models, the variance is now different. However, the parametric relations have not changed.

$$
\begin{aligned}
\phi & =e^{-\kappa}, \\
S_{d}^{2} & =\sigma^{2} \frac{1-\phi^{2}}{2 \kappa}=\sigma^{2} \frac{1-e^{-2 \kappa}}{2 \kappa} .
\end{aligned}
$$

Substituting these relations into (6.15), we obtain

$$
\sigma^{2} \frac{e^{-\kappa t}-e^{-\kappa(s+t)}}{\kappa} x_{0}+\sigma^{2} \frac{e^{-\kappa(t-s)}-e^{-\kappa(t+s)}}{2 \kappa} \theta
$$

which is the continuous covariance CIR model. Thus we have a discrete representation of the CIR model so that the mean and covariance function are equal for all sampling times with a parametric relationship between between the discrete and continuous parameters.

The least squares estimate of $\phi$ is the value that minimizes the residual sum of squares

$$
\phi_{0}=\underset{\phi}{\operatorname{argmin}} \sum_{t=1}^{N} \frac{\left(x_{t}-\phi x_{t-1}\right)^{2}}{x_{t}+\theta}
$$


Figure 3: The solid line represents the continuous-time covariance function and the dashed line represents the discrete-time covariance function. They are related through the sampling interval $t$.

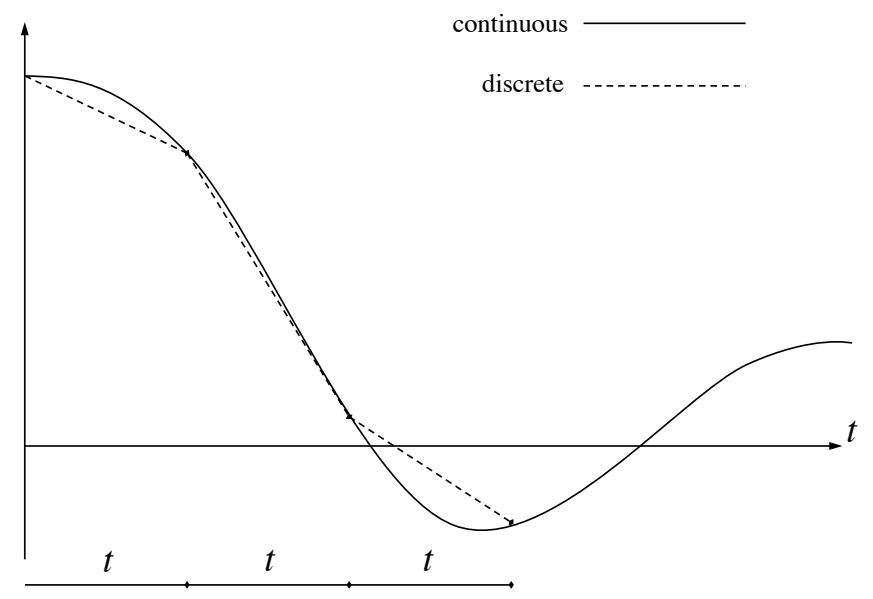

and the variance is taken as

$$
S_{d}^{2}=\frac{\sum_{t=1}^{N} \frac{\left(x_{t}-\phi_{0} x_{t-1}\right)^{2}}{x_{t}+\theta}}{N} .
$$

\section{Time series analysis results.}

In addition providing point estimates of the parameters, we are interested in computing appropriate standard deviations to provide interval estimates. Essentially, we have two ways of computing the appropriate standard deviations.

The first requires us to compute the matrix of second derivatives of the log-likelihood function. This method is not feasible in the case of the CIR model. The second method, the method we implement, is to use (6.2) to set the parameters $\kappa, \theta$ and $\sigma$ at their maximum likelihood estimates and then use Monte Carlo simulations to generate repeated samples of the same size as the observed data. Each simulated sample uses a different random number generator seed so that they can be treated as independent and identically (i.i.d.) distributed replications. For each of these simulated samples, we compute the maximum likelihood estimates as we did for the observed Treasury data. We then effectively have a set of i.i.d. draws from the sampling distribution of the maximum likelihood estimates. 
Table 2: Maximum likelihood estimates for the entire data set.

\begin{tabular}{cccc}
\hline & 1-month & 3-month & 6-month \\
\hline mean & 0.00084 & 0.00114 & 0.0019 \\
std. dev. & 0.0006 & 0.00068 & 0.0010 \\
\hline$\kappa$ & 0.0650 & 0.0331 & 0.015 \\
& & & \\
$\theta$ & 0.0024 & 0.0036 & 0.0057 \\
& & & \\
$\sigma$ & 0.1010 & 0.0712 & 0.0499 \\
\hline
\end{tabular}

We generate 5000 simulations of the 1-month, 3-month and 6-month Treasury bill. Each simulation is of the same length of the collected data. For each simulated process, we calculate the maximum likelihood estimators. In the figure below, we plot three simulated CIR processes along with the observed 3-month interest rate. The blue dotted line is the observed interest rate.

Figure 4: A comparison of observed and simulated interest rates.

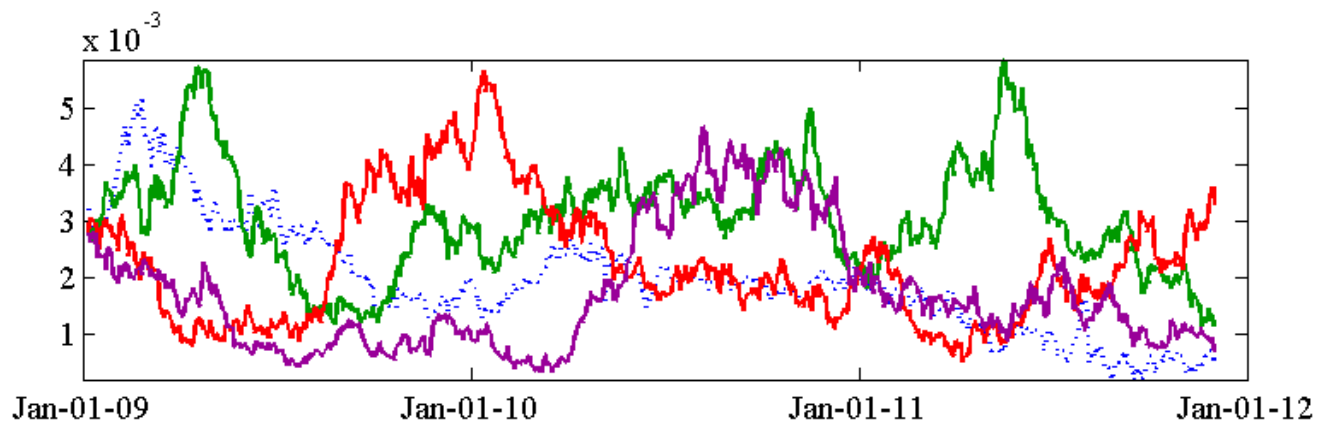

Assuming each generated process is independent and identically distributed, we then have independent samples of the parameters $(\kappa, \theta, \sigma)$. Displayed in the table below are sample mean and sample standard deviation for the maximum likelihood estimate of each parameter.

Alternatively, we can provide point estimates in the following manner. Let $N$ denote the number of data points for each bond and suppose we write $N=n \cdot n$ for some natural number $n$. That is, we suppose that we have $n$ data groups each containing $n$ values for each set of bond data. We calculate the maximum likelihood estimates of the parameters for each of the $n$ groups, 
Table 3: Maximum likelihood estimates for the simulated CIR processes.

\begin{tabular}{cccc}
\hline & 1-month & 3-month & 6-month \\
\hline mean & 0.00084 & 0.00114 & 0.0019 \\
std. dev. & 0.0006 & 0.00068 & 0.0010 \\
\hline \multirow{2}{*}{$\kappa$} & 0.0851 & 0.0418 & 0.0204 \\
& $(0.0794)$ & $(0.0563)$ & $(0.0284)$ \\
$\theta$ & 0.0034 & 0.0015 & 0.0106 \\
& $(0.0071)$ & $(0.0389)$ & $(0.0185)$ \\
$\sigma$ & 0.1007 & 0.0680 & 0.0424 \\
& $(0.0097)$ & $(0.0157)$ & $(0.0170)$ \\
& & & \\
\hline
\end{tabular}

resulting in the samples

$$
\left\{\left(\kappa_{i}, \theta_{i}, \sigma_{i}\right)\right\}_{i=1}^{n}
$$

As an estimate for each parameter, we take

$$
\bar{\kappa}=\frac{1}{n} \sum_{i=1}^{n} \kappa_{i}, \quad \bar{\theta}=\frac{1}{n} \sum_{i=1}^{n} \theta_{i}, \quad \bar{\sigma}=\frac{1}{n} \sum_{i=1}^{n} \sigma_{i}
$$

We estimate the standard deviation for each estimate by taking the sample standard deviation and dividing by $\sqrt{n}$. Note that for our parameter set $N=729=27^{2}$.

In the table below, we have the mean and estimated error for each parameter by taking each set of bond data as 27 groups of 27 data points.

Table 4: Parameter estimates using the data grouping approach.

\begin{tabular}{cccc}
\hline & 1-month & 3-month & 6-month \\
\hline mean & 0.00084 & 0.00114 & 0.0019 \\
std. dev. & 0.0006 & 0.00068 & 0.0010 \\
\hline$\kappa$ & 0.0892 & 0.0975 & 0.0845 \\
& $(0.0238)$ & $(0.0265)$ & $(0.0299)$ \\
$\theta$ & 0.0013 & 0.0014 & 0.0026 \\
& $(0.0005)$ & $(0.0003)$ & $(0.0004)$ \\
$\sigma$ & 0.0582 & 0.0549 & 0.0574 \\
& $(0.0089)$ & $(0.0053)$ & $(0.0100)$ \\
& & &
\end{tabular}


By comparing table 6.3 with table 6.4 , one can see that the estimates for each parameter in the 1-month and 3-month bonds are quite close, with the error estimates in the grouping approach slightly smaller. Since the estimates obtained using the grouping approach are obtained directly from the data, we are inclined to consider them more accurate.

If we assume that the CIR model is a very accurate description of the term structure, then given the results in table (6.4), we take the midpoint of each interval estimate (the interval being the point estimate \pm one standard deviation).

\subsection{CROSS-SECTION ANALYSIS}

Here we discuss parameter estimation using the term structure, i.e., the yield curve for estimating the parameters of the CIR model.

For each day $t_{i}, i=0, \ldots, n$, the vector $\left(R_{i}^{j}\right)_{j=1}^{M}$ where

$$
R_{i}^{j}:=\left(R_{t_{i}}, t_{i}+\tau_{j}\right)
$$

denotes the return at the end of day $t_{i}$ for maturity $\tau_{j}$. Notice that in (6.15) the maturities are fixed.

We write $R_{i}$ for the vector $\left(R_{i}^{j}\right)_{j=1}^{M}$ which we refer to as the cross-section observed on day $t_{i}$, thus we have $N=n+1$ cross-sections.

We consider two types of cross-sectional estimation, the first is a daily cross-section estimation and the second is a composite cross-section estimation.

\section{Daily Cross-Sections.}

For first procedure we used to fit the CIR model day-to-day cross-section of returns. That is, for each $i=1,2, \ldots, n$ we solve the problem

$$
\left(\sigma_{i}, \kappa_{i}, \theta, r_{i}\right)=\underset{\sigma, \kappa, \theta, r}{\operatorname{argmin}} \sum_{j=1}^{M}\left[R_{i}^{j}-A\left(\tau_{j}\right)-r B\left(\tau_{j}\right)\right]^{2}
$$

and restricting $\kappa, \theta, \sigma^{2}$ and $r$ to be non-negative. It is important to note that for each sampling time $t_{i}$, we obtain different estimates of $\kappa_{i}, \theta_{i}, \sigma_{i}$ and $r_{i}$. 


\section{Composite Cross-Sections.}

In this procedure, we perform a least-squares fit to all the cross-sections of returns, keeping the parameters $\theta, \kappa$ and $\sigma$ constant for all observation days. That is, solve the problem

$$
(\sigma, \kappa, \theta, \mathbf{r})=\underset{\sigma, \kappa, \theta, \mathbf{r}}{\operatorname{argmin}} \sum_{i=0}^{n} \sum_{j=1}^{M}\left[R_{i}^{j}-A\left(\tau_{j}\right)-r_{i} B\left(\tau_{j}\right)\right]^{2} .
$$

The term $\mathbf{r}$ denotes the entire parameter set $\left\{r_{i}\right\}$. Again we restrict $\kappa, \theta$ and $\sigma$ to be non-negative in addition to $r_{i}$ for each $i$.

Alternatively, we can approach the problem this way. If we have estimates for

Unlike the time-series approach discussed in the previous section, the cross-section approach, particular the composite cross-section approach (6.17) allow to us to provide an estimation of a realization of the theoretical short rate using the actual realization of the term structure.

We continue with the results of the cross-section analysis. The main issue in implementation is whether the cross-sections should be performed daily or performed over composite cross-sections.

For a daily cross-section analysis, we estimate $\kappa, \theta, \sigma$ and $r$ using (6.16). Thus, for each time $t_{i}$, we obtain the estimates $\kappa_{i}, \theta_{i}, \sigma_{i}$ and $r_{i}$. The results of the analysis are displayed in the table below. Given the large number of observations, for an interval estimate we take the mean estimate plus/minus two standard errors.

Table 5: Results of the daily cross-section analysis.

\begin{tabular}{cccccc}
\hline Parameter & 1st quartile & Median & 3rd quartile & Mean & Mean $\pm 2 \cdot \mathrm{SE}$ \\
\hline$\kappa$ & 0.0241 & 0.0351 & 0.0447 & 0.0326 & $(0.0321,0.0332)$ \\
$\theta$ & 0.0010 & 0.0019 & 0.0029 & 0.0072 & $(0.0066,0.0078)$ \\
$\sigma$ & 0.0436 & 0.0462 & 0.0504 & 0.0477 & $(0.0474,0.0480)$ \\
\hline
\end{tabular}

Using the estimated short rate obtained from the daily cross-section estimation, we performed a maximum likelihood estimation as we did in the time series section, the results are listed in the table below.

For composite cross-section analysis, $\kappa, \theta, \sigma$ as well as $\mathbf{r}=\left\{r_{i}\right\}_{i=0}^{N}$ are estimated over $N$ cross-sections of the yield curve. Thus, for each group of $N$ data points there will be one estimate 
Table 6: Parameter estimates for the estimated short rate.

\begin{tabular}{cc}
\hline & $\left\{r_{i}\right\}$ \\
\hline mean & 0.0011 \\
std. dev. & 0.00062 \\
\hline$\kappa$ & 0.0432 \\
& $(0.0201)$ \\
$\theta$ & 0.0016 \\
& $(0.0013)$ \\
$\sigma$ & 0.0261 \\
& $(0.0100)$ \\
\end{tabular}

for each of $\kappa, \theta$ and $\sigma$ but an estimate of the short rate over for each of the $N$ data points. We performed the analysis separately for data groups in 27 cross-sections. That is, we have perform the estimation described by (6.17) over the 27 data groups, each containing 27 cross-sections of the yield curve. Thus, we get the samples

$$
\left\{\left(\kappa_{i}, \theta_{i}, \sigma_{i}, \mathbf{r}\right)\right\}_{i=1}^{27}, \quad \text { where } \quad \mathbf{r}=\left\{r_{i}\right\}_{i=1}^{27}
$$

In the figure below, we compare the estimated short rates using the daily and composite crosssection methods. The large variation in the composite short rate is due to the fact that the numerical optimization performed over each of the 27 data groups is done with respect to many parameters.

\subsection{REMARKS ON ESTIMATION METHODS}

It is frequently cited in the research literature that despite the many popular features of various short rate models, only $70-80 \%$ of the term structure can be described by a short rate model [22]. Thus, while more attention could be directed towards accurate estimates of the CIR model parameters, we are satisfied with the estimates obtained in this chapter.

With the estimates obtained in our analyses we have sufficient accuracy to continue with further numerical aspects of the mortgage prepayment problem. For the rest of the thesis, for any 
Figure 5: Comparison of the cross-section estimated short rate.
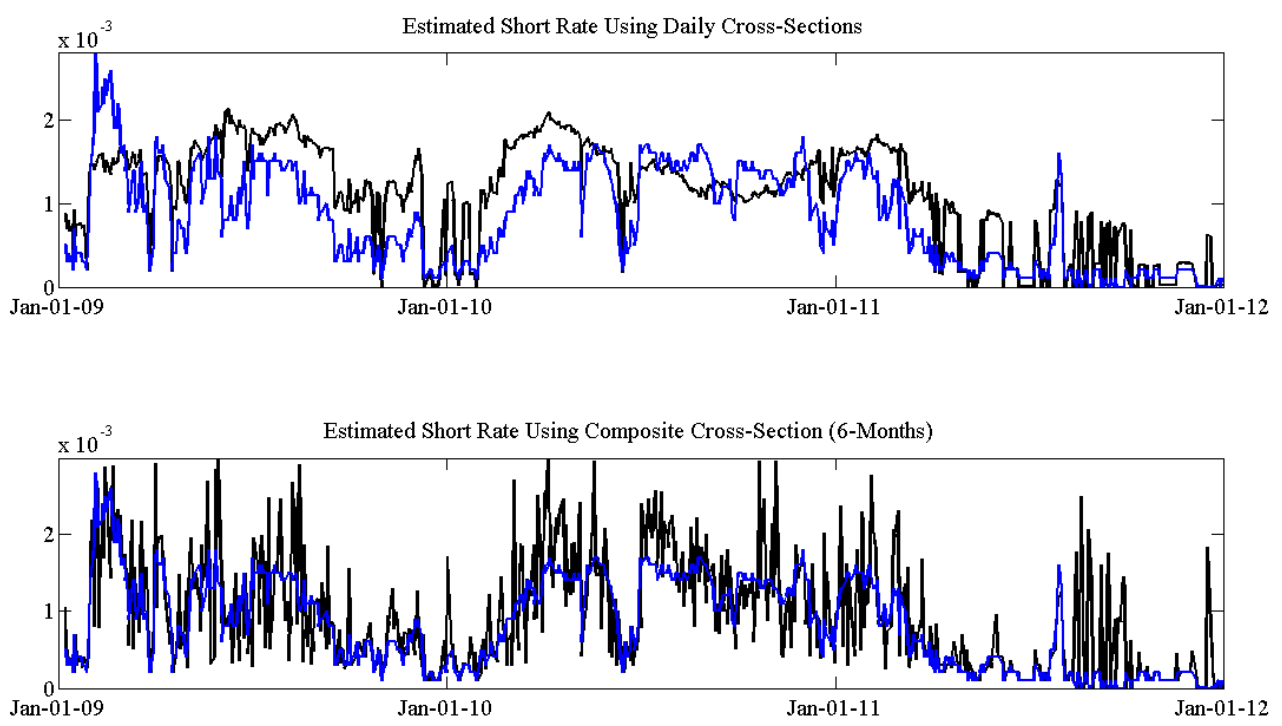

Table 7: Parameter estimates using the composite method.

\begin{tabular}{cc}
\hline & $\left\{r_{i}\right\}$ \\
\hline mean & 0.00088 \\
std. dev. & 0.00095 \\
\hline$\kappa$ & 0.0402 \\
& $(0.0262)$ \\
$\theta$ & 0.0022 \\
& $(0.00023)$ \\
$\sigma$ & 0.02036 \\
& $(0.0095)$ \\
\hline
\end{tabular}

computation based on the values of the parameters, we will assume

$$
\kappa=0.09(0.02), \quad \theta=0.0013(0.0005), \quad \sigma=0.0582(0.009) .
$$

Notice then that the no-flux parameter, integral to both the variational and free boundary approaches, is then estimated by

$$
b=\frac{2 \kappa \theta}{\sigma^{2}}=0.067(0.021)
$$


As we mentioned earlier, a popular feature of the CIR model is that if $b=2 \kappa \theta / \sigma^{2} \geq 1$ and positive $r_{0}$, the short rate $r_{t}$ will never hit zero. On the other hand, if $b=2 \kappa \theta / \sigma^{2}<1$, then it will occasionally hit zero. In the observed term structure data, both the 1-month and 3-month rates have multiple days where a zero level rate was recorded. Thus, a value of $b$ less than one is to be expected.

Remark 6.5.1. ( $i$ ) With the presence of zero rates, other estimation methods such as the method of least squares, which one obtains from the discretized CIR equation by

$$
\frac{r_{i+1}-r_{i}}{\sqrt{r_{i} \Delta t}}=\kappa \theta \frac{\sqrt{\Delta t}}{\sqrt{r_{i}}}-\kappa \sqrt{r_{i} \Delta t}+\sigma e_{i}, \quad e_{1}, e_{2}, \ldots, e_{n} \text { are (i.i.d.) } N(0,1)
$$

become quite problematic to implement.

(ii) For data sets without zero rates, using the least squares estimates for initial points in the numerical optimization for the maximum likelihood estimates did not always result in convergence. It appears that this is largely due to the fact that over small data sets ( less than 100 data points), the least square estimate for $\kappa$ is often exceedingly large. This problem of rapid divergence could be dealt with by using a scaled version of the modified Bessel function

$$
I_{q}^{*}(2 \sqrt{u v})=I_{q}(2 \sqrt{u v}) \exp (-2 \sqrt{u v})
$$




\subsection{NUMERICAL ASPECTS}

In this chapter we will investigate aspects of a numerical solution of the variational inequality corresponding to (5.10):

$$
\min \left\{W_{\tau}-\mathcal{L} W+P_{\tau}, \quad W\right\}=0 \text { on }(0, \infty)^{2}, \quad W(\cdot, 0)=0 .
$$

where

$$
\mathcal{L}=:=\frac{1}{2} \sigma^{2} r \partial_{r r}+\kappa(r-\theta) \partial_{r}-r \quad \text { and } \quad P(r, \tau):=(c-r)\left[1-e^{-c r}\right] c^{-1}
$$

In Section 4.1, we established that the free boundary $\Gamma:=\{(r, \tau) \mid \tau>0, r=x(\tau)>0\}$ is well-defined.

\subsection{THE FREE BOUNDARY NEAR EXPIRATION}

In this section we discuss the behavior of the free boundary as $\tau \searrow 0$. We consider the simplified system of (5.2)

$$
\begin{cases}W_{\tau}-\frac{\sigma^{2}}{2} r W_{r r}=\tau(r-c) & \text { if } 0<x(\tau)<r<+\infty, \tau>0 \\ W(x(\tau), \tau)=0, \quad W_{r}(x(\tau), \tau)=0 & \text { if } \tau>0, x(\tau)>0, \\ \lim _{r \searrow 0} r^{b} W_{r}(r, \tau)=0 & \text { if } \tau>0, x(\tau)=0, \\ x(0)=c, \quad W(\cdot, 0)=0 & \end{cases}
$$


Similar to what we did in Section 5.1, using the smooth fit conditions and the first equation in (7.1), we can evaluate $W_{r} r(x, \tau)$ as

$$
W_{r r}(x, \tau)=-\frac{2}{\sigma^{2}}(c-x) \tau .
$$

We now write the differential equation in the simplified system as

$$
W_{\tau}-\frac{\sigma^{2} c}{2} W_{r r}=\tau(r-c)+\frac{\sigma^{2}}{2}(r-c) W_{r r} .
$$

Near $(c, 0)$, we have

$$
W_{\tau}-\frac{\sigma^{2} c}{2} W_{r r} \approx \tau((r-c))
$$

so we're left with the standard parabolic free boundary problem

$$
\begin{cases}U_{\tau}-\frac{\sigma^{2}}{2} c U_{r r}=\tau(r-c) & \text { if } 0<s(\tau)<r<+\infty, \tau>0 \\ U(s(\tau), \tau)=0, \quad U_{r}(s(\tau), \tau)=0 & \text { if } \tau>0, s(\tau)>0 \\ s(0)=c, \quad U(\cdot, 0)=0 & \end{cases}
$$

Near the point $(c, 0)$, we have

$$
W(r, \tau) \approx U(r, \tau), \quad x(\tau) \approx s(\tau)
$$

where $(U, s)$ is the solution of system (7.2). Let $s(\tau)$ be the free boundary of system (7.2), then as $\tau \searrow 0$

$$
s(\tau) \approx c-\sigma \alpha \sqrt{c \tau}
$$

where $\alpha$ is the solution of the following equation

$$
\left(\alpha^{5}+10 \alpha^{3}\right) e^{\frac{\alpha^{2}}{4}} \int_{-\infty}^{\alpha} e^{-\frac{\eta^{2}}{4}} d \eta=16-16 \alpha^{2}-2 \alpha^{2} .
$$


Letting $z=\frac{\sqrt{2}}{\sigma} \frac{c-r}{\sqrt{c \tau}}, \quad s(\tau)=c-\sigma \alpha \sqrt{c \tau}, \quad U(r, \tau)=\tau^{\frac{5}{2}} g(z)$ we obtain the ordinary free boundary problem for $g$

$$
\begin{gathered}
g^{\prime \prime}(z)+\frac{z}{2} g^{\prime}(z)-\frac{5}{2} g(z)=\beta z, \quad-\infty<z<\alpha \\
g>0 \text { in }(\infty, \alpha], \quad g=0 \text { in }(\alpha, \infty) \\
g(\alpha)=g^{\prime}(\alpha)=0, \quad g(z)=o\left(z^{5}\right) \text { as } z \rightarrow-\infty
\end{gathered}
$$

We find particular and homogeneous solutions to equation for $g$ :

$$
g_{p}(z)=-\frac{\beta}{2} z \quad g_{h}(z)=z^{5}+20 z^{3}+60 z
$$

and the special function solution

$$
\psi(z)=\left(z^{5}+20 z^{3}+60 z\right) \int_{-\infty}^{z} e^{-\eta^{2} / 4} d \eta+2\left(z^{4}+18 z^{2}+32\right) e^{-z^{2} / 4}
$$

A general solution to the free boundary problem is then

$$
g(z)=g_{p}(z)+c_{1} g_{h}(z)+c_{2} \psi(z)
$$

The boundary conditions imply

$$
\begin{aligned}
-\beta z / 2+c_{2} \psi(z) & =0 \\
\beta / 2+c_{s} \psi^{\prime}(z) & =0 \\
c_{1} & =0 .
\end{aligned}
$$

Eliminating $c_{2}$ we obtain $\psi(\alpha)-\alpha \psi^{\prime}(\alpha)=0$ which is the equation (7.4). Uniqueness of the root follows by considering the function $f(\alpha)=\psi(\alpha)-\alpha \psi^{\prime}(\alpha)$ and the easily verified facts:

$$
f(0)=-16, \quad f(+\infty)=+\infty, \quad f^{\prime}(\alpha)>0 .
$$

A numerical calculation gives

$$
\alpha \approx 0.409209 \ldots
$$


Figure 6: The asymptotic free boundary for $\tau \in[0,0.05]$

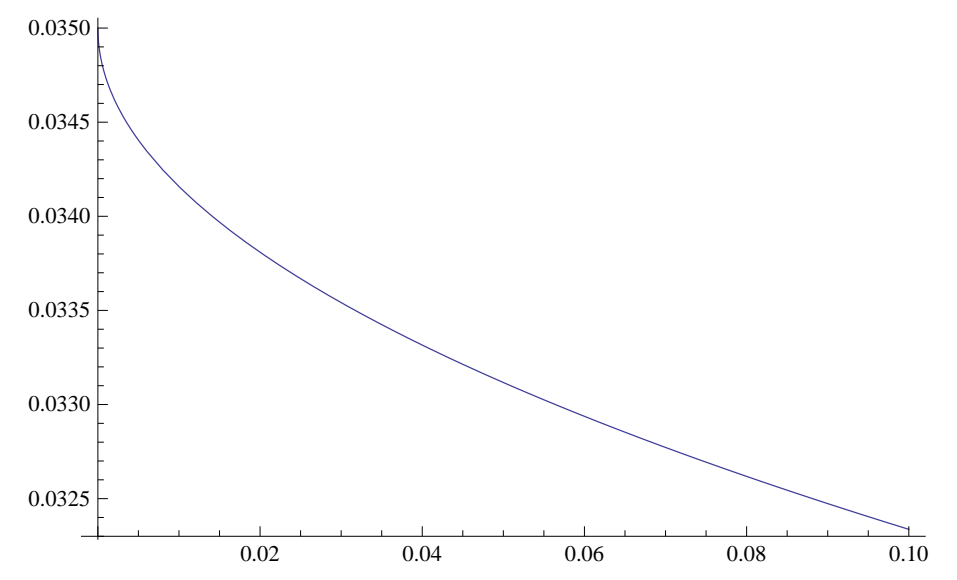

\subsection{TRANSFORMATIONS TO CANONICAL FORMS}

In Section 5.1, we formulated the obstacle problem (2.1) as a free boundary problem (5.2) with the smooth-fit condition

$$
W(x(\tau), \tau)=0, \quad W_{r}(x(\tau), \tau)=0 \quad \text { if } \quad \tau>0, x(\tau)>0
$$

and the no-flux boundary condition

$$
\lim _{r \searrow 0} r^{b} W_{r}(r, \tau)=0 \quad \text { if } \quad \tau>0, x(\tau)=0
$$

The solution of (2.1), together with $x$ is a solution of the free boundary problem (5.2), which is typically how the free boundary is solved. Several technical difficulties that must be addressed when solving this problem. Namely, the operator $\mathcal{L}$ is unbounded at $r=\infty$ and degenerate at $r=0$, which numerically may cause stability problems for certain schemes. In the following section, we introduce transformations to address the technical difficulties.

Here we introduce a few transformations that transfer the operator $\partial_{\tau}-\mathcal{L}$ into dimensionless canonical forms.

1. Dimensionless Form. Introduce the dimensionless quantities

$$
\hat{\tau}=c \tau, \quad \hat{r}=\frac{r}{c}, \quad \hat{W}=\frac{c W}{m}, \quad \hat{\sigma}=\frac{\sigma}{c}, \quad \hat{\kappa}=\frac{\kappa}{c}, \quad \hat{\theta}=\frac{\theta}{c} .
$$


Then the variational inequality (2.1) can be written as

$$
\min \left\{\partial_{\hat{\tau}} \hat{W}-\hat{\mathcal{L}} \hat{W}+(1-\hat{r})\left(1-e^{-\hat{\tau}}\right), \hat{W}\right\}=0
$$

where $\hat{\mathcal{L}}$ denotes the dimensionless form of $\mathcal{L}$ and is given by $\hat{\mathcal{L}}=\frac{1}{2} \hat{\sigma}^{2} \hat{r} \partial_{\hat{r} \hat{r}}+\hat{\kappa}(\hat{\theta}-\hat{r}) \partial_{\hat{r}}-\hat{r}$.

Hence, we obtain exactly the same problem except $\hat{m}=1$ and $\hat{c}=1$. Dropping the $\hat{\text {, we }}$ can assume, without loss of generality, that $m=1, c=1$. We proceed to transform the partial differential equation $\partial_{\tau} W-\mathcal{L} W$ into a suitable canonical form.

2. The First Canonical Form. We now introduce the constants

$$
b=\frac{2 \kappa \theta}{\sigma^{2}}, \quad \hat{\mu}:=\frac{c\left(\kappa+\sqrt{\kappa^{2}+2 \sigma^{2}}\right)}{\sigma^{2}}, \quad \hat{\gamma}=\frac{\sqrt{\kappa^{2}+2 \sigma^{2}}}{c}, \quad \hat{D}=\frac{\sigma^{2}}{2 c \sqrt{\kappa^{2}+2 \sigma^{2}}}
$$

Make the change of variables $(\hat{r}, \hat{\tau}, \hat{W}) \rightarrow(y, s, w)$ by

$$
y=e^{\hat{\gamma} \hat{\tau}} \hat{r}, \quad s=\hat{D}\left(e^{\hat{\gamma} \hat{\tau}}-1\right), \quad w(y, s)=e^{-\hat{\mu}(\hat{r}+\hat{\kappa} \hat{\theta} \hat{\tau})} \hat{W}(\hat{r}, \hat{\tau})
$$

One can check that

$$
\left(\partial_{\hat{\tau}}-\hat{\mathcal{L}}\right) \hat{W}=\frac{1}{2} \hat{\sigma}^{2} e^{\hat{\mu} \hat{r}+(\hat{\gamma}+\hat{\kappa} \hat{\theta} \hat{\mu}) \hat{\tau})}\left\{w_{s}-\left(y w_{y y}+b w_{y}\right)\right\}
$$

Thus, the variational inequality (2.1) can be written as

$$
\min \left\{w_{s}-y w_{y y}-b w_{y}-f, \quad w\right\}=0 \text { in }(0, \infty)^{2}
$$

where

$$
f(y, s)=\left.\frac{2(\hat{r}-1)\left(1-e^{-\hat{\tau}}\right)}{\hat{\sigma}^{2} e^{\hat{\mu} r+(\hat{\mu} \hat{\kappa} \hat{\theta}+\hat{\gamma}) \hat{\tau}}}\right|_{\hat{r}=\frac{y}{1+s / \hat{D}}, \hat{\tau}=\frac{\ln (1+s / \hat{D})}{\hat{\gamma}}} .
$$

Note that both $w$ and $f$ decay exponentially fast in terms of the original variable $(r, \tau)$. In the new variable, they decay exponentially fast as $y \rightarrow \infty$, and algebraically fast as $s \rightarrow \infty$.

The no-flux condition $\lim _{r \searrow 0} r^{b} W_{r}=0$ in the new variables becomes $\lim _{y \searrow 0} y^{b} w_{y}=0$.

3. The Second Canonical Form. To remove the the non-constant coefficient $y$ of $w_{y y}$, we introduce the new variable $z=2 \sqrt{y}$. The partial differential operator can now be written as

$$
y \partial_{y y}+b \partial_{y}=\partial_{z z}+\frac{2 b-1}{z} \partial_{z}, \quad \text { and } \quad y^{b} \partial_{y}=2^{1-2 b} z^{2 b-1} \partial_{z}
$$


Thus, the variational problem can be written as

$$
\min \left\{w_{s}-w_{z z}-\frac{2 b-1}{z} w_{z}-f, \quad w\right\}=0 .
$$

where

$$
f(z, s)=\left.\frac{2(\hat{r}-1)\left(1-e^{-\hat{\tau}}\right)}{\hat{\sigma}^{2} e^{\hat{\mu} r+(\hat{\mu} \hat{\kappa} \hat{\theta}+\hat{\gamma}) \hat{\tau}}}\right|_{\hat{r}=\frac{z^{2} / 2}{1+s / \hat{D}}, \hat{\tau}=\frac{\ln (1+s / \hat{D})}{\hat{\gamma}}},
$$

The transformed problems (7.6) and (7.8) can be implemented using standard upwind/downwind schemes for parabolic equations.

\subsection{DISCRETIZATION AND IMPLEMENTATION}

Here we describe numerical methods for solving the problems given by (7.6) and (7.8). Note that the equations in both canonical forms can be written in a divergence form

$$
y \partial_{y y} w+b \partial_{y} w=y^{1-b}\left(y^{b} w_{y}\right)_{y} \quad \text { and } \quad w_{z z}+(2 b-1) z^{-1} w_{z}=z^{1-2 b}\left(z^{2 b-1} w_{z}\right)_{z}
$$

1. The First Canonical Form. Let $\left\{y_{i}\right\}$ denote the mesh points and denote by $w_{i}$ the value of $w$ at $y_{i}$, i.e., $w_{i}=w\left(y_{i}, s\right)$. As usual, we denote $y_{i+1 / 2}=\frac{1}{2}\left(y_{i}+y_{i+1}\right)$ and $y_{i-1 / 2}=y_{(i-1)+1 / 2}$.

At $y=y_{i}$, the operator $y \partial_{y y}+b \partial_{y}=y^{1-b}\left(y^{b} w_{y}\right)_{y}$ may be discretized as follows:

$$
y w_{y y}+b w_{y} \approx \frac{y_{i}^{1-b}}{y_{i+1 / 2}-y_{i-1 / 2}}\left(\frac{y_{i+1 / 2}^{b}\left(w_{i+1}-w_{i}\right)}{y_{i+1}-y_{i}}-\frac{y_{i-1 / 2}^{b}\left(w_{i}-w_{i-1}\right)}{y_{i}-y_{i-1}}\right) .
$$

The no-flux boundary condition $\lim _{y \searrow 0} y^{b} w_{y}=0$ maybe simply approximated by $w_{1}=w_{0}$.

We introduce a grid

$$
\begin{gathered}
y_{i}:=0+\left(i-\frac{1}{2}\right) \Delta y, \quad i=1,2, \ldots, \quad \Delta y:=\frac{\left(y_{\max }\right)}{J}, \\
\mu:=\frac{\Delta s}{(\Delta y)^{2}}, \\
\alpha_{i}=y^{b-1}, \quad \beta_{i \pm \frac{1}{2}}=\left(y_{i \pm \frac{1}{2}}\right)^{b} \\
y_{i \pm \frac{1}{2}}:=0+\left(i \pm \frac{1}{2}-\frac{1}{2}\right) \Delta y, \quad i=2,3, \ldots, J-1 .
\end{gathered}
$$


for $J$ a given positive integer, so that $y_{1}=\Delta \eta / 2$ and $y_{J}=y_{\max }-\Delta y / 2$; no quantities are evaluated at the singular end point $y=0$. The numerical approximation to $w\left(y_{i}, s_{n}\right)=w\left(y_{i}, n \Delta s\right)$, having set $s_{n}:=n \Delta s, \quad n=0,1,2, \ldots$, will be denoted by $w_{i}^{n}$.

We derive an explicit, implicit and an averaged Crank-Nicolson type scheme. For $w>0$, i.e. The explicit scheme:

$$
\alpha_{i}\left(w_{i}^{n+1}-w_{i}^{n}\right)=\mu\left[\beta_{i+\frac{1}{2}}\left(w_{i+1}^{n}-w_{i}^{n}\right)-\beta_{i-\frac{1}{2}}\left(w_{i}^{n}-w_{i-1}^{n}\right)\right]+(\Delta s) f_{i}^{n}
$$

The implicit scheme:

$$
\alpha_{i}\left(w_{i}^{n+1}-w_{i}^{n}\right)=\mu\left[\beta_{i+\frac{1}{2}}\left(w_{i+1}^{n+1}-w_{i}^{n+1}\right)-\beta_{i-\frac{1}{2}}\left(w_{i}^{n+1}-w_{i-1}^{n+1}\right)\right]+(\Delta s) f_{i}^{n+1}
$$

The averaged scheme:

$$
\begin{array}{r}
\alpha_{i}\left(w_{i}^{n+1}-w_{i}^{n}\right)=\frac{\mu}{2}\left[\beta_{i+\frac{1}{2}}\left(w_{i+1}^{n+1}-w_{i}^{n+1}\right)-\beta_{i-\frac{1}{2}}\left(w_{i}^{n+1}-w_{i-1}^{n+1}\right)\right] \\
+\frac{\mu}{2}\left[\beta_{i+\frac{1}{2}}\left(w_{i+1}^{n}-w_{i}^{n}\right)-\beta_{i-\frac{1}{2}}\left(w_{i}^{n}-w_{i-1}^{n}\right)\right]+\frac{\Delta s}{2}\left(f_{i}^{n}+f_{i}^{n+1}\right) .
\end{array}
$$

The exponential decay of $w$ as $y \rightarrow \infty$ and the no-flux boundary condition as $y \rightarrow 0^{+}$can be written as

$$
\lim _{z \searrow 0} z^{2 b-1} \frac{\partial w}{\partial z}=0 \quad \text { and } \quad \lim _{z \nearrow \infty} z^{2 b-1} \frac{\partial w}{\partial z}=0
$$

Thus, with second order accuracy, we write

$$
\begin{array}{r}
\alpha_{1}\left(w_{1}^{n+1}-w_{1}^{n}\right)=\mu\left[\beta_{3 / 2}\left(w_{2}^{n+1}-w_{1}^{n+1}\right)+\beta_{3 / 2}\left(\left(w_{2}^{n}-w_{1}^{n}\right)\right)\right], \\
\alpha_{J}\left(w_{J}^{n+1}-w^{n+1}\right)=\mu\left[-\beta_{J-1 / 2}\left(w_{J}^{n+1}-w_{J-1}^{n+1}\right)+\beta_{J-1 / 2}\left(\left(w_{J}^{n}-w_{J-1}^{n}\right)\right)\right] .
\end{array}
$$

The equations (7.21) and (7.15) represent a system of $J$ linear equations in $J$ unknowns. Introduce the diagonal matrix

$$
A:=\operatorname{diag}\left(\alpha_{1}, \alpha_{2}, \ldots, \alpha_{J}\right)
$$


the symmetric tridiagonal matrix

$$
B:=\left(\begin{array}{cccccc}
\beta_{3 / 2} & -\beta_{3 / 2} & \ldots & \ldots & \ldots & \ldots \\
-\beta_{3 / 2} & \beta_{3 / 2}+\beta_{5 / 2} & -\beta_{5 / 2} & \ldots & \ldots & \ldots \\
\ldots & -\beta_{5 / 2} & \ldots & \ldots & \ldots & \ldots \\
\ldots & \ldots & \ldots & \ldots & \ldots & \ldots \\
\ldots & \ldots & \ldots & \ldots & \beta_{J-3 / 2}+\beta_{J-1 / 2} & -\beta_{J-1 / 2} \\
\ldots & \ldots & \ldots & \ldots & -\beta_{J-1 / 2} & \beta_{J-1 / 2}
\end{array}\right)
$$

and the vectors

$$
w^{n}:=\left(w_{1}^{n}, w_{2}^{n}, \ldots, w_{J}^{n}\right)^{T}, \quad f^{n}:=\left(f_{1}^{n}, f_{2}^{n}, \ldots, f_{J}^{n}\right)^{T} .
$$

The Crank-Nicolson system (7.21) may now be written as

$$
A\left(w^{n+1}-w^{n}\right)=-\mu B\left(w^{n+1}-w^{n}\right)+\frac{\Delta s}{2}\left(f^{n}+f^{n+1}\right),
$$

or

$$
(A+\mu B) w^{n+1}=(A-\mu B) w^{n}+\frac{\Delta s}{2}\left(f^{n}+f^{n+1}\right) .
$$

We now verify that the scheme is well-defined, i.e., that the symmetric matrix $A+\mu B$ in 7.17 is invertible. By direct calculation, we have

$$
v^{T} B v=\sum_{i=1}^{J-1} \beta_{i+\frac{1}{2}}\left(v_{i+1}-v_{i}\right)^{2}
$$

for any vector

$$
v:=\left(v_{1}, v_{2}, \ldots, v_{J}\right)^{T}
$$

Thus, the symmetric matrix $B$ is positive semidefinite, and its only null (kernel) vector is

$$
e:=(1,1, \ldots, 1)^{T}
$$

At each time step $n$ we calculate the free boundary according to

$$
Y^{n}=\sup \left\{\eta_{i} \mid w_{i}^{n}=0\right\}
$$


here $Y(s)$ denotes the free boundary and $Y^{n} \approx Y(n \Delta s)$.

2. The Second Canonical Form. The operator $w_{z z}+(2 b-1) z^{-1} w_{z}=z^{1-2 b}\left(z^{2 b-1} w_{z}\right)_{z}$ may be discretized at the mesh point $z=z_{i}$ as

$$
w_{z z}+\frac{2 b-1}{z} w_{z} \approx \frac{z_{i}^{1-2 b}}{z_{i+1 / 2}-z_{i-1 / 2}}\left(\frac{z_{i+1 / 2}^{2 b-1}\left(w_{i+1}-w_{i}\right)}{z_{i+1}-z_{i}}-\frac{z_{i-1 / 2}^{2 b-1}\left(w_{i}-w_{i-1}\right)}{z_{i}-z_{i-1}}\right) .
$$

In the second canonical form, the exponential decay of $w$ as $z \rightarrow \infty$ and the no-flux boundary condition as $z \rightarrow 0^{+}$can be written as

$$
\lim _{y \searrow 0} y^{b} \frac{\partial w}{\partial y}=0 \quad \text { and } \quad \lim _{y \nearrow \infty} y^{b} \frac{\partial w}{\partial y}=0
$$

We define the grid

$$
\begin{gathered}
z_{i}:=0+\left(i-\frac{1}{2}\right) \Delta z, \quad i=1,2, \ldots, \quad \Delta z:=\frac{\left(z_{\max }\right)}{J}, \\
\mu:=\frac{\Delta s}{(\Delta z)^{2}}, \\
\alpha_{i}=z^{1-2 b}, \quad \beta_{i \pm \frac{1}{2}}=\left(z_{i \pm \frac{1}{2}}\right)^{2 b-1}, \\
z_{i \pm \frac{1}{2}}:=0+\left(i \pm \frac{1}{2}-\frac{1}{2}\right) \Delta z, \quad i=2,3, \ldots, J-1 .
\end{gathered}
$$

for $J$ a given positive integer, so that $z_{1}=\Delta \eta / 2$ and $z_{J}=y_{\max }-\Delta y / 2$; no quantities are evaluated at the singular end point $z=0$. The numerical approximation to $w\left(z_{i}, s_{n}\right)=w\left(z_{i}, n \Delta s\right)$, having set $s_{n}:=n \Delta s, \quad n=0,1,2, \ldots$, will be denoted by $w_{i}^{n}$.

We derive an explicit, implicit and an averaged Crank-Nicolson type scheme. For $w>0$, i.e. The explicit scheme:

$$
\alpha_{i}\left(w_{i}^{n+1}-w_{i}^{n}\right)=\mu\left[\beta_{i+\frac{1}{2}}\left(w_{i+1}^{n}-w_{i}^{n}\right)-\beta_{i-\frac{1}{2}}\left(w_{i}^{n}-w_{i-1}^{n}\right)\right]+(\Delta s) f_{i}^{n} .
$$

The implicit scheme:

$$
\alpha_{i}\left(w_{i}^{n+1}-w_{i}^{n}\right)=\mu\left[\beta_{i+\frac{1}{2}}\left(w_{i+1}^{n+1}-w_{i}^{n+1}\right)-\beta_{i-\frac{1}{2}}\left(w_{i}^{n+1}-w_{i-1}^{n+1}\right)\right]+(\Delta s) f_{i}^{n+1} .
$$

The averaged scheme:

$$
\begin{array}{r}
\alpha_{i}\left(w_{i}^{n+1}-w_{i}^{n}\right)=\frac{\mu}{2}\left[\beta_{i+\frac{1}{2}}\left(w_{i+1}^{n+1}-w_{i}^{n+1}\right)-\beta_{i-\frac{1}{2}}\left(w_{i}^{n+1}-w_{i-1}^{n+1}\right)\right] \\
+\frac{\mu}{2}\left[\beta_{i+\frac{1}{2}}\left(w_{i+1}^{n}-w_{i}^{n}\right)-\beta_{i-\frac{1}{2}}\left(w_{i}^{n}-w_{i-1}^{n}\right)\right]+\frac{\Delta s}{2}\left(f_{i}^{n}+f_{i}^{n+1}\right) .
\end{array}
$$


where in this canonical form

$$
f(z, s)=\left.\frac{2(\hat{r}-1)\left(1-e^{-\hat{\tau}}\right)}{\hat{\sigma}^{2} e^{\hat{\mu} r+(\hat{\mu} \hat{\kappa} \hat{\theta}+\hat{\gamma}) \hat{\tau}}}\right|_{\hat{r}=\frac{z^{2}}{4(1+s / \hat{D})}, \hat{\tau}=\frac{\ln (1+s / \hat{D})}{\hat{\gamma}}},
$$

Again, in the case of the Crank-Nicolson scheme we have the system of equations

$$
A\left(w^{n+1}-w^{n}\right)=-\mu B\left(w^{n+1}-w^{n}\right)+\frac{\Delta s}{2}\left(f^{n}+f^{n+1}\right),
$$

i.e.,

$$
(A+\mu B) w^{n+1}=(A-\mu B) w^{n}+\frac{\Delta s}{2}\left(f^{n}+f^{n+1}\right) .
$$

Remark 7.3.1. Because of the degeneracy, one or both the first rows of the matrix (7.16) become singular when $\Delta y$ is small and vanish as $\Delta y \rightarrow 0$. Therefore, ill-conditioned matrices do occur for typical reasons, namely that their determinant is very small. Simple preconditioning strategies, such as using a Choleski factorization addresses this issue.[20, 28, 33].

Figure 7: Approximation of the Optimal Termination Boundary

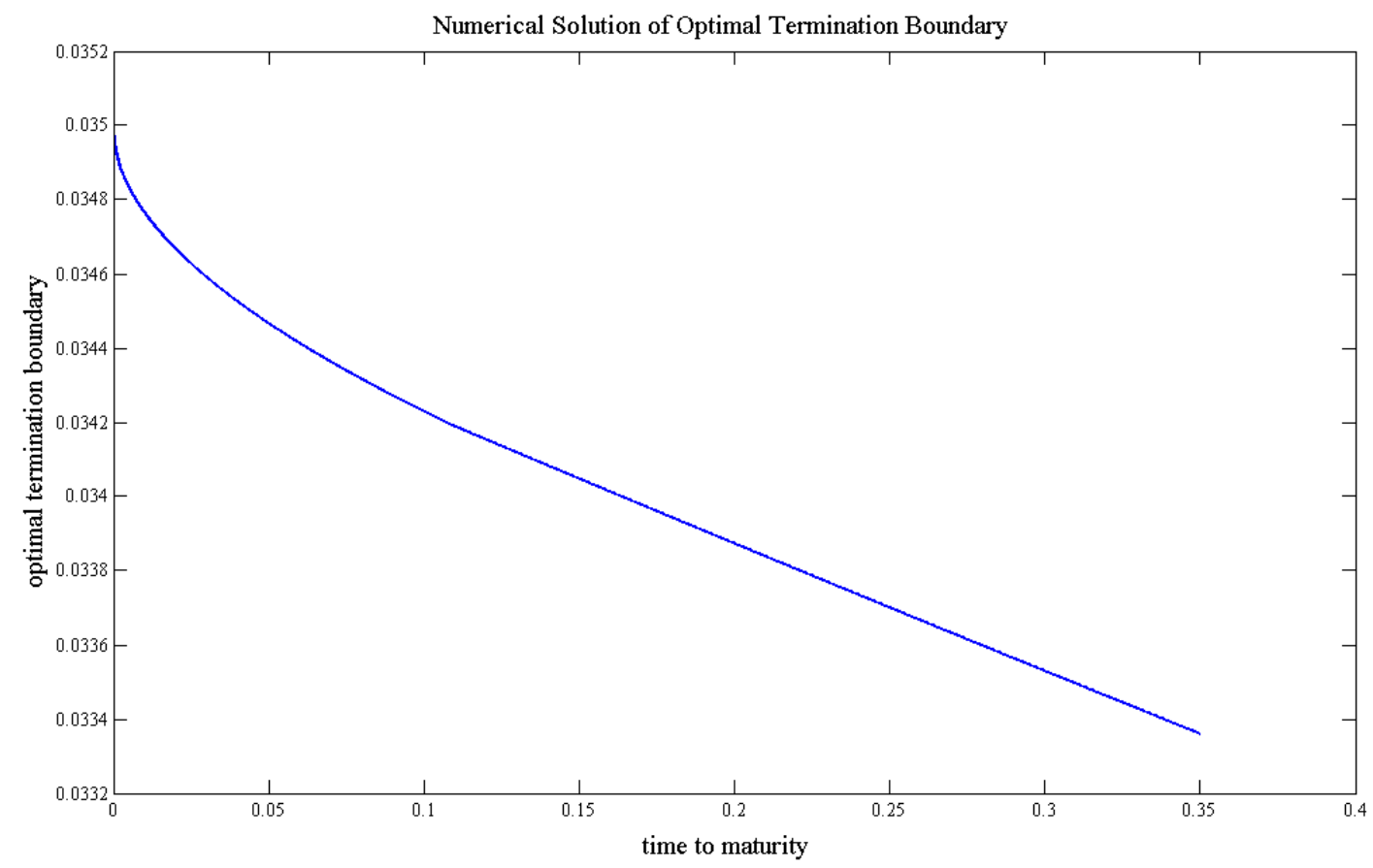




\subsection{CONCLUSION}

In this thesis, we model the prepayment incentive option of a fixed rate mortgage. We assume the decision to prepay is primarily related to the term structure, i.e., the market return rate, which we assume to be modeled by the Cox-Ingersoll-Ross process. If the market return rate is low enough, the mortgagor has a decision to exercise the prepayment option and prepay the mortgage.

As a simplification, we assume there are no transaction costs associated with prepayment. Typ-

ically, there is a fee required for prepayment, which typically ranges anywhere from one-half to two percentage points over the outstanding balance. Since transaction costs are typically proportional to the outstanding balance, the issue of including a fee can be addressed in the following manner.

Let $\phi$ represent the outstanding balance quota that is required as a prepayment fee. The value $V$ at interest rate $r_{t}$ at time $t$ would then satisfy

$$
\min \left\{V_{t}+\mathcal{L} V+m,(1+\phi) B-V\right\}=0
$$

A fixes rate mortgage is equivalent to an American call on an amortizing bond, so by refinancing, the mortgagor is attempting to determine the best time to call the bond, i.e., exercise the call option. Our focus is restricted to the concrete example of finding the "where and when" optimal prepayment should occur, that is when the first time the market interest rate is at a level that refinancing is possible. This optimal rate represented by the free boundary in our formulation of the problem.

Most mortgage prepayments come in the form of a new mortgage used to repay the existing one, that is, refinancing. In addition to a lower mortgage rate, the mortgagor usually has a choice as to the length of the new contract. Let's assume that the mortgagor has two choices, refinance with a new mortgage at the same term $T$ or refinance with a new mortgage having half the term, 
i.e., $T / 2$. This would correspond to the usual 30 -year and 15 -year refinancing options offered by banks. Thus the prepayment decision now involves optimizing over a type of compound option. The mortgagor is now picking the refinancing policy that minimizes their expected net present value. 


\section{APPENDIX A}

\section{PROOFS OF ANCILLARY RESULTS}

\section{A.1 PROOF OF PROPOSITION 2.2.2}

We have $A(0) F_{t}+B(0)=0$, so $A(0)=0, B(0)=0$, since $Z_{T}^{T}=1$. Next, for any fixed $T$, as a stochastic process of $t$, we have, writing $\tau=T-t$,

$$
\begin{gathered}
d Z_{t}^{T}=Z_{t}^{T}\left(R_{t}^{T} d t+\sigma_{t}^{T} d W_{t}\right) \\
\sigma_{t}^{T}:=\sigma_{t} A(\tau), \quad R_{t}^{T}:=A(\tau) \mu_{t}+A^{2}(\tau) \sigma_{t}^{2} / 2-A^{\prime}(\tau) F_{t}-B^{\prime}(\tau)
\end{gathered}
$$

By the fundamental theorem of asset pricing, there exist processes $\left\{r_{t}, \lambda_{t}\right\}$ such that $R_{t}^{T}=$ $r_{t}+\lambda_{t} \sigma_{t}^{T}$, i.e.

$$
B^{\prime}(\tau)+A^{\prime}(\tau) F_{t}+r_{t}+\left(\lambda_{t} \sigma_{t}-\mu_{t}\right) A(\tau)-A^{2}(\tau) \sigma_{t}^{2} / 2=0
$$

As $A(0)=0$ and $A \not \equiv 0$, there exists $\tau_{0}>0$ such that $A\left(\tau_{0}\right) A^{\prime}\left(\tau_{0}\right) \neq 0$. Dividing (A.1) by $A(\tau)$ and differentiating the resulting equation with respect to $\tau$ we obtain

$$
\left(\frac{B^{\prime}(\tau)}{A(\tau)}\right)^{\prime}+\left(\frac{A^{\prime}(\tau)}{A(\tau)}\right)^{\prime} F_{t}-\frac{A^{\prime}(\tau)}{A^{2}(\tau)} r_{t}-A^{\prime}(\tau) \frac{\sigma_{t}^{2}}{2}=0
$$

Dividing by $A^{\prime}(\tau)$ and differentiating the resulting equation with respect to $\tau$ we obtain

$$
\left[\frac{1}{A^{\prime}(\tau)}\left(\frac{B^{\prime}(\tau)}{A(\tau)}\right)^{\prime}\right]^{\prime}+\left[\frac{1}{A^{\prime}(\tau)}\left(\frac{A^{\prime}(\tau)}{A(\tau)}\right)^{\prime}\right]^{\prime} F_{t}+\frac{2 A^{\prime}(\tau)}{A^{3}(\tau)} r_{t}=0
$$


Setting $\tau=\tau_{0}$, we find that $r_{t}=c_{0}+c_{1} F_{t}$ for some constants $c_{0}$ and $c_{1}$. Substituting this relation back to (A.2) at $\tau=\tau_{0}$ we find that $\sigma_{t}^{2}=\sigma_{0}+\sigma_{1} F_{t}$ for some constants $\sigma_{0}$ and $\sigma_{1}$. Substituting these relations back to (A.1) at $\tau=\tau_{0}$ we find that $\lambda_{t} \sigma_{t}-\mu_{t}=k_{0}+k_{1} F_{t}$ for some constants $k_{0}, k_{1}$. In addition, as $F_{t}$ is non-constant, (A.1) implies that $A$ and $B$ are solutions of $A^{\prime}=\sigma_{1}^{2} A^{2} / 2-k_{1} A-c_{1}$ and $B^{\prime}=\sigma_{0}^{2} A_{2} / 2-k_{0} A-c_{0}$, respectively.

Note that $c_{1} \neq 0$ since otherwise we would have $A \equiv 0$. Thus $F_{t}=\left(r_{t}-c_{0}\right) / c_{1}$. This means that we can use $r_{t}$ as the factor, so we can assume that $c_{0}=0, c_{1}=1$. In summary, for a one factor ATSM model, there are constants $\sigma_{0}, \sigma_{1}, k_{0}, k_{1}$ and a stochastic process $\left\{\lambda_{t}\right\}$ such that

$$
\begin{aligned}
& d r_{t}=\hat{\mu}_{t} d t+\sigma_{t} d \hat{W}_{t}, \quad \hat{\mu}_{t}=k_{0}+k_{1} r_{t}, \sigma_{t}^{2}=\sigma_{0}+\sigma_{1} r_{t}, \quad \hat{W}_{t}=W_{t}+\int_{0}^{t} \lambda_{s} d s \\
& Z_{t}^{T}=e^{A(T-t) r_{t}+B(T-t)}, \quad d Z_{t}^{T}=Z_{t}^{T}\left[r_{t} d t+A(T-\tau) \sigma_{t} d \hat{W}_{t}\right] \\
& A^{\prime}=\frac{\sigma_{1}^{2}}{2} A^{2}-k_{1} A-1, \quad A(0)=0, \quad B(\tau)=\int_{0}^{\tau}\left[\frac{\sigma_{0}^{2}}{2} A^{2}-k_{0} A\right] .
\end{aligned}
$$

This completes the proof of the proposition.

\section{A.2 THE FUNDAMENTAL SOLUTION AND DERIVATION OF THE TRANSITION DENSITY FUNCTION}

The solution of the initial boundary value problem, for $w=w(r, \tau)$,

$$
\begin{cases}w_{\tau}-\mathcal{L} w=f & \text { in }(0, \infty)^{2}, \\ w(\cdot, 0)=w_{0}(\cdot) & \text { on }(0, \infty) \times\{0\}, \\ \lim _{r \searrow 0} r^{b} w_{r}(r, \cdot) d \tau=h(d \tau) & \text { in measure on }\{0\} \times(0, \infty)\end{cases}
$$

can be expressed by Green's formula using the fundamental solution, $p=p(r ; \varrho, t)$, of

$$
\begin{cases}p_{t}=\mathcal{L}^{*} p & \text { in }(0, \infty)^{2}, \\ p(r ; \cdot, 0)=\delta(\cdot-r) & \text { on }(0, \infty) \times\{0\} \\ \lim _{\varrho \searrow 0}\left[(\varrho p)_{\varrho}-b p\right]=0 & \text { on }\{0\} \times(0, \infty)\end{cases}
$$


where $\delta(\cdot-r)$ is the Dirac measure concentrated at $r$ and $\mathcal{L}^{*}$ is the adjoint of $\mathcal{L}$ given by

$$
\mathcal{L}^{*} p=2^{-1} \sigma^{2}(\varrho p)_{\varrho \varrho}-\kappa[(\theta-\varrho) p]_{\varrho}-\epsilon \varrho p
$$

with the default $\epsilon=1$. If we let $\epsilon=0$, the corresponding solution $p(r ; \cdot, t)$ is the probability density of the random variable $r_{t}$ under the condition $r_{0}=r$ in the CIR model. Here we want to find explicitly the fundamental solution, to explain the boundary condition on $\{0\} \times(0, \infty)$ in (A.3).

Introduce a transformation from $(\varrho, t, p)$ to $(y, \varsigma, P)$ by

$$
\begin{gathered}
y=l(t) \varrho, \quad \varsigma=[1-l(t)] \sigma^{2}(2 \gamma)^{-1}, \quad p(r ; \varrho, t)=l(t) e^{\lambda(r-\varrho+\kappa \theta t)} P(r ; y, \varsigma), \\
l(t):=e^{-\gamma t}, \quad \gamma=\sqrt{\kappa^{2}+2 \epsilon \sigma^{2}}, \quad \lambda=\frac{\kappa+\gamma}{\sigma^{2}}, \quad \mu=\frac{\gamma-\kappa}{\sigma^{2}} .
\end{gathered}
$$

Then $P$ solves the following canonical equation with only one parameter:

$$
P_{\varsigma}=\left[(y P)_{y}-b P\right]_{y}, \quad P(r ; \cdot, 0)=\delta(\cdot-r), \quad \lim _{y \searrow 0}\left[(y P)_{y}-b P\right]=0 .
$$

Using the Laplace transform (in the $y$ variable) one can derive that

$$
\begin{aligned}
\hat{P}(r ; \xi, \varsigma) & :=\int_{0}^{\infty} e^{-\xi y} P(r ; y, \varsigma) d y=[1+\varsigma \xi]^{-b} e^{-r \xi /(1+\varsigma \xi)}, \\
P(r ; y, \varsigma) & =\frac{e^{-(r+y) / \varsigma}}{\varsigma}\left(\frac{y}{\varsigma}\right)^{b-1} \Phi\left(\frac{r y}{\varsigma^{2}}\right), \\
\Phi(x) & :=\frac{1}{2 \pi \mathbf{i}} \int_{1-\mathbf{i} \infty}^{1+\mathbf{i} \infty} z^{-b} e^{z+x / z} d z=\sum_{k=0}^{\infty} \frac{x^{k}}{k ! \Gamma(k+b)} \\
& =\frac{x^{\frac{1-b}{2}}}{2 \pi \mathbf{i}} \int_{1-\mathbf{i} \infty}^{1+\mathbf{i} \infty} t^{-b} e^{\sqrt{x}(t+1 / t)} d t=\frac{x^{\frac{1}{4}-\frac{b}{2}} e^{2 \sqrt{x}}}{2 \sqrt{\pi}}\left(1+O\left(x^{-\frac{1}{2}}\right)\right)
\end{aligned}
$$

where $\Gamma$ is the Gamma function. Note that $x \Phi_{x x}+b \Phi_{x}-\Phi=0$ and $\Phi(x)=x^{\frac{1-b}{2}} I_{b-1}(2 \sqrt{x})$ where $I_{\nu}(z)$ is the modified first kind Bessel function of order $\nu$. Therefore,

$$
\begin{aligned}
p(r ; \varrho, t) & =\varrho^{b-1} \varsigma^{-b} e^{-[\mu+l / \varsigma] r-[\lambda+l / \varsigma] \varrho-[\gamma-\kappa] b t / 2} \Phi\left(r \varrho l \varsigma^{-2}\right), \\
p_{1}(r ; t) & :=\lim _{\varrho \searrow 0} \varrho^{1-b} p(r ; \varrho, t)=\varsigma^{-b} e^{-[\mu+l / \varsigma] r-[\gamma-\kappa] b t / 2} \Gamma(b)^{-1} \\
p_{2}(r ; t) & :=\lim _{\varrho \searrow 0} \varrho^{-b}\left[(\varrho p)_{\varrho}-b p\right]=p_{1}(r ; t)\left(r l b^{-1} \varsigma^{-2}-l \varsigma^{-1}-\lambda\right) .
\end{aligned}
$$




\section{A.3 DERIVATION GREEN'S FORMULA AND BOND PRICES}

Green's formula is obtained by integrating $\left[w_{t}(\varrho, t)-\mathcal{L} w\right] p(r ; \varrho, \tau-t)=f p$ over $\left[\varepsilon_{1}, 1 / \varepsilon_{1}\right] \times$ $\left[0, \tau-\varepsilon_{2}\right]$ for small positive $\varepsilon_{1}$ and $\varepsilon_{2}$, using integration by parts and sending first $\varepsilon_{1} \searrow 0$ and then $\varepsilon_{2} \searrow 0$. The limit exists if appropriate growth conditions are imposed. Using the asymptotic behavior of $p(r ; \varrho, \tau)$ as $\varrho \searrow 0$ and as $\varrho \rightarrow \infty$, one can prove the following:

Lemma A.3.1 (Green's Representation). Let $h(d \tau)$ be a measure on $(0, \infty), w_{0}$ and $f$ be a locally bounded functions satisfying $w(r), f(r, \tau)=O\left(e^{\lambda r}\right)$ as $r \rightarrow \infty$. Then under the growth condition $w=O\left(e^{\lambda r}\right)$ as $r \rightarrow \infty$ and $w=o\left(r^{-b}\right)$ as $r \searrow 0$, problem (A.3) admits a unique solution, and the solution is given by the Green representation

$$
w(r, \tau)=\int_{0}^{\infty} w_{0}(\varrho) p(r ; \varrho, \tau) d \varrho+\int_{0}^{\tau} \int_{0}^{\infty} f(\varrho, t) p(r ; \varrho, \tau-t) d \varrho d t-\frac{\sigma^{2}}{2} \int_{0}^{\tau} p_{1}(r ; \tau-t) h(d t)
$$

where $p$ is the fundamental solution and $p_{1}(r ; t)=\lim _{\varrho \searrow 0} \varrho^{1-b} p(r ; \varrho, t)$, given in (A.5),(A.6).

Remark A.3.2. When $b \in(0,1)$, Dirichlet boundary values on $\{0\} \times(0, \infty)$ can also be assigned for the equation $w_{\tau}-\mathcal{L} w=0$ on $(0, \infty)^{2}$; the corresponding fundamental solution is to replace

$\Phi(x)$ by $\tilde{\Phi}(x):=x^{\frac{1-b}{2}} I_{1-b}(2 \sqrt{x})$. For more general theory, see Oleînik [35] and the references in [41].

In mathematical finance, a $T$-bond is a guaranteed unit payment at a future time $T$. Under the CIR term structure model, if the current time is $t$ and the short rate is $r_{t}=r$, then the risk neutral price, $Z(r, \tau)$, of the $T$-bond with time-to-maturity $\tau=T-t$ is the solution of (2.1) with $w_{0} \equiv 1, h \equiv 0, f \equiv 0$. The yield $Y(r, \tau)$ is defined as $-\tau^{-1} \log Z(r, \tau)$. Using Green's formula and the known Laplace transform, we immediately obtain the following:

Corollary A.3.3. Under a current short rate $r_{t}=r$, the risk-neutral price of a $T$-bond is

$$
\begin{aligned}
Z(r, \tau) & =\int_{0}^{\infty} p(r ; \varrho, \tau) d \varrho=e^{\lambda r+\lambda \kappa \theta \tau} \hat{P}\left(r ; \lambda l^{-1}, \tau\right) \\
& =\exp \left(-\frac{2[1-l] r}{(\gamma+\kappa)+(\gamma-\kappa) l}-\frac{b \tau}{\lambda}-b \ln \left[1-\frac{1-l}{\gamma \lambda}\right]\right)
\end{aligned}
$$


where $\tau=T-t, l=e^{-\gamma \tau}, \gamma=\sqrt{\kappa^{2}+2 \sigma^{2}}, b=2 \kappa \theta / \sigma^{2}$, and $\lambda=(\kappa+\gamma) / \sigma^{2}$. Consequently, the yields of short term bonds and long term bonds have the following behavior:

$$
\begin{aligned}
& Y(r, \tau)=r+\frac{\kappa \tau}{2}(\theta-r)+O\left(\tau^{2}\right) \quad \text { as } \tau \searrow 0, \\
& Y(r, \tau)=\frac{2 \kappa}{\kappa+\sqrt{\kappa+2 \sigma^{2}}} \theta+O\left(\tau^{-1}\right) \quad \text { as } \tau \rightarrow \infty .
\end{aligned}
$$

Remark A.3.4. As expected, the CIR model is affine in the sense that yields are affine functions of the factor, which is taken to be the short rate $r$ in the model. The mean reversion of the short rate implies that the yield of short term bonds deviates from the short rate in the direction towards the $\theta$ value. However, yields of very long term bonds are strictly smaller than $\theta$.

\section{A.4 PROOF OF LEMMA 2.1.1}

Set $\eta=\lambda+\mu$ and make the change of variables from $(r, G(r))$ to $(x, K(x))$ by

$$
x=\eta r, \quad K(x)=e^{\mu x / \eta} G\left(x \eta^{-1}\right), \quad \text { i.e. } \quad G(r)=e^{-\mu r} K(\eta r) .
$$

Then $K(\cdot)$ satisfies Kummer's equation (confluent hypergeometric equation) [27]

$$
x K_{x x}(x)+(b-x) K_{x}(x)-a K(x)=0 .
$$

Note that $a$ and $b$ given by (2.2) satisfy $b>a>0$. Two linear independent solutions of Kummer's equation are Kummer's (1837) function, $M(a, b ; z)$, and Tricomi's (1947) function, $U(a, b ; z)$ :

$$
\begin{aligned}
M(a, b ; x) & =\frac{\Gamma(b)}{\Gamma(a) \Gamma(b-a)} \int_{0}^{1} e^{x t} t^{a-1}(1-t)^{b-a-1} d t & & (b>a>0) \\
U(a, b ; x) & =\frac{1}{\Gamma(a)} \int_{0}^{\infty} e^{-x t} t^{a-1}(1+t)^{b-a-1} d t & & (a>0) .
\end{aligned}
$$

When $b>a>0, M(a, b ; \cdot)$ (writing it as $M(\cdot)$ ) is an entire function having the properties

$$
M(0)=1, \quad M^{\prime}(0)=a b^{-1}, \quad M^{\prime}>0 \text { on } \mathbb{R}, \quad M(z) \sim e^{z} z^{a-b} \Gamma(b) \Gamma(a)^{-1} \text { as } z \rightarrow \infty
$$


When $a>0$, the function $U(a, b ; \cdot)$ (writing it as $U(\cdot))$ is analytic in $(0, \infty)$ having properties $U_{z}(z) \sim-z^{-b} \Gamma(b) \Gamma(a)^{-1}$ as $z \searrow 0, \quad U>0>U_{z}$ on $(0, \infty), \quad U(z) \sim z^{-a}$ as $z \rightarrow \infty$

The assertion of the lemma thus follows by taking

$$
G_{1}(r)=\eta^{b-1} \Gamma(a) \Gamma(b)^{-1} e^{-\mu r} U(a, b ; \eta r), \quad G_{2}(r)=e^{-\mu r} M(a, b ; \eta r) .
$$




\section{APPENDIX B}

\section{EXTENSIONS OF THE PROBLEM}

In this chapter, we briefly discuss possible extensions of the mortgage prepayment problem that will productively contribute to the advancement of the research.

\section{B.1 GENERAL AFFINE TERM STRUCTURE MODELS}

So far, the mortgage prepayment option has been examined under various short rate models, the Vasicek and CIR models. The decision to prepay is based on the term structure and the Vasicek and CIR models don't capture all aspects of it.

A more general theory of the mortgage prepayment option therefore requires a more general theory of the term structure. Namely, the general affine term structure.

In the case of multidimensional ATSMs, the price $Z_{t}^{T}$ of the $T$-bond at time $t$ is assumed to satisfy

$$
\log \frac{1}{Z_{t}^{T}}=L_{0}(T-t)+L_{1}(T-t) F_{t}^{1}+\cdots+L_{n}(T-t) F_{t}^{n} \forall 0 \leqslant t
$$

where $L_{0}(\cdot), L_{1}(\cdot), L_{n}(\cdot)$ are differentiable functions and $F_{t}:=\left\{\left(F_{t}^{1}, \ldots, F_{t}^{n}\right)\right\}$ is an Ito process [14]. In two or three factors, one can more accurately describe aspects of the yield curve such as level, shape and steepness.

Moreover, under fairly general assumptions on the Ito processes, one can derive hedging arguments for pricing interest rate derivatives. A benefit in addition to the statistical accuracy is that the 
corresponding Black-Scholes equation in this model is still linear. For the mortgage prepayment problem there will be difficulties to surmount such as boundary conditions for the state space in addition to the difficulty of the free boundary problem.

\section{B.2 AN INTEGRAL EQUATION APPROACH}

Integral equation methods are becoming increasingly useful in studying free boundary problems for early termination contracts $[39,9]$. Thus, one prospect of future work would be to implement an integral equation method for the problem analyzed in this thesis. To develop an integral equation method we need a Green's function.

Consider then the first canonical form (7.6)

$$
\min \left\{w_{s}-y w_{y y}-b w_{y}-f, \quad w\right\}=0 \text { in }(0, \infty)^{2}
$$

For the operator $\partial_{s}-y \partial_{y y}-b \partial_{y}$, the associated Green's function $G(x ; y, s)$ is the solution of the system

$$
\begin{cases}p_{s}=(y p)_{y y}-(b p)_{y} & \text { on }(0, \infty)^{2}, \\ \lim _{y \searrow 0}\left\{y p_{y}+(1-b) y\right\}=0 & \text { on }\{0\} \times(0, \infty), \\ p(x ; y, 0)=\delta(x-y) & \end{cases}
$$

here $\delta$ is the Dirac delta function.

One can check that the solution is given by

$$
p(x ; y, s)=\frac{y^{(b-1) / 2} e^{-y / s}}{s x^{(b-1) / 2} e^{x / s}} I_{b-1}\left(\frac{2 \sqrt{x y}}{s}\right) .
$$

Where $I_{q}(z)$ is the modified Bessel function of the first kind of order $q$. Additionally, $I_{q}(z)$ has the integral representation

$$
I_{q}(z)=\frac{z^{q / 2}}{\sqrt{\pi} \Gamma(q+1 / 2)} \int_{-1}^{1}\left(1-t^{2}\right)^{q-1 / 2} \cosh (z t) d t, \quad q>-\frac{1}{2} .
$$


and the asymptotic behavior

$$
I(z)=\left(\frac{z}{2}\right)^{q} \frac{\left[1+O\left(z^{2}\right)\right]}{\Gamma(q+1)} \text { as } z \searrow 0, \quad I_{q}(z)=\frac{e^{z}}{\sqrt{2 \pi z}}\left\{1-\frac{O(1)}{z}\right\} \quad \text { as } z \rightarrow \infty
$$

Note that $p$ has the following properties:

$$
\lim _{y \searrow 0} y^{1-b} p=\frac{e^{-x / s}}{\Gamma(b) s^{b}}, \quad \lim _{y \searrow 0} y^{-b}\left[(y p)_{y}-b p\right]=\frac{e^{-x / s}}{\Gamma(b) s^{b}}\left(\frac{x}{b s^{2}}-\frac{1}{s}\right) .
$$

Now suppose $w$ is a solution of the initial boundary value problem

$$
w_{s}=y w_{y y}+b w_{y}+f \text { in }(0, \infty)^{2}, \quad \lim _{y \searrow 0}\left(-y^{b} w_{y}\right)=h, \quad w(\cdot, 0)=g(\cdot) .
$$

Then for $0<\varepsilon<s$ and $x>0$, since $p=O\left(y^{b-1}\right)$ and $(y p)_{y}-b p=O\left(y^{b}\right)$ as $y \searrow 0$, we have

$$
\begin{aligned}
& \int_{0}^{s-\varepsilon} \int_{0}^{\infty} p(x ; y, s-t) f(y, t) d y d t=\int_{0}^{s-\varepsilon} \int_{0}^{\infty} p\left[w_{t}-y w_{y y}-b w_{y}\right] d y d t \\
= & \int_{0}^{\infty}[p(x ; y, \varepsilon) w(y, s-\varepsilon)-p(x ; y, t) w(y, 0)]+\int_{0}^{s-\varepsilon} h(t) \frac{e^{-x /(s-t)}}{\Gamma(b)(s-t)^{b}} d t
\end{aligned}
$$

Sending $\varepsilon \searrow 0$ we then obtain

$$
w(x, s)=\int_{0}^{\infty} p(x ; y, t) g(y) d y+\int_{0}^{s} \frac{h(s-t) e^{-x / t}}{\Gamma(b) t^{b}}+\int_{0}^{s} \int_{0}^{\infty} p(x ; y, s-t) f(y, t) d y d t .
$$




\section{BIBLIOGRAPHY}

[1] Alobaidi, G. \& Mallier, R. 2006 Installment options close to expiry. Journal of Applied Mathematics and Stochastic Analysis, (Art. ID 60824), 1-9.

[2] BJORK, A. 2004 Arbitrage theory in continuous time, 2nd ed.. Oxford. Oxford University Press.

[3] Black, F. \& SChOles, M. 1973 The pricing of options and corporate liabilities. The J. of Political Economy, 81, 637-659.

[4] Brennan, M. \& Schwatrz, E. 1977 The valuation of American put options. Journal of Finance, 32, 449-462.

[5] Brown, R. H. \& Schaefer, S. M. 1994 The term structure of real interest rates and the Cox, Ingersoll, and Ross model.

[6] Buser, S. A. \& Hendershott, P. H. 1984 Pricing default-free fixed rate mortgages. Housing Finance Rev., 3, 405-429.

[7] Carr, P., Jarrow, R., Myneni, R. 1992 Alternative characterizations of American put options. Mathematical Finance, 2 (1992), 87-106.

[8] Chan, K. C., Karolyi, G. A., Longstaff F. A. \& Sanders, A. B. 1992 An empirical comparison of alternative models of the short-term interest rate. The Journal of Finance, XLVII, 1209-1227.

[9] Chen, X. \& ChADAm, J. 2003 Analytic and numerical approximations for the early exercise boundary for American put options. Dynamics of Continuous, Discrete and Impulsive Systems 10, 649-657.

[10] Chen, X. \& Chadam, J. 2006 A mathematical analysis of the optimal boundary for American put options. SIAM Journal on Mathematical Analysis 38, 1613-1641.

[11] Chen, X., Chadam, J., Jiang, L., \& Zheng, W. 2008 Convexity of the exercise boundary of the American put option on a zero dividend asset. Mathematical Finance 18, 185-197.

[12] Chen, X., Cheng, H. \& Chadam, J. 2011 Non-convexity of the optimal exercise boundary for an american put option on a dividend-paying asset. preprint 
[13] Chen, X., Cheng, H. \& Chadam, J. 2011 Far-from-expiry behavior of the optimal exercise boundary for an American put option on a dividend-paying asset. Proceeding of the American Mathematical Society 139, 273-282.

[14] Chen, X. \& HuAng, T. 2009 A theoretical and empirical analysis of linear term structure models.

[15] Cox, J. C., Ingersoll, J. E., \& Ross, S. A. 1985 A Theory of The Term Structure of Interest Rates. Econometrica 53(2), March 385-407.

[16] Crandall, M. G., Ishit, H. \& Lions, P.-L. 1992 User's guid to viscosity solutions of second order partial differential equations Bulletin of American Mathematical Society 27, $1-67$.

[17] Duffie, D. 2001 Dynamic Asset Pricing Theory. New Jersey. Princeton University Press.

[18] Duffie, D. \& KAn, R. 1994 Multi-Factor Term Structure Models. Philosophical Transactions of the Royal Society of London 347, 577-586.

[19] DufFIE, D. \& KAN, R. 1996 A yield-factor model of interest rates. Mathematical Finance 6, 379-406.

[20] Ehrhardt, M. \& Mickens, R. E. 2008 A fast, stable and accurate numerical method for the Black-Scholes equation of American options. Int. J. Theor. Appl. Finance 11, 471-501.

[21] FELler, W. 1951 Two singular diffusion problems Annals of Mathematics 2, 173-182.

[22] Filipović, D. 2009 Term-Structure Models: A graduate course. Springer Finance, SpringerVerlag, Berlin.

[23] Friedman, A. 1964 Partial Differential Equations of Parabolic Type. New Jersey. PrenticeHall.

[24] Friedman, A. 1976 Analyticity of the free boundary for the Stefan problem. Archive for Rational Mechanics and Analysis 61, 97-125.

[25] Friedman, A. 1982 Variational Principles and Free Boundary Problems. New York. John Wiley \& Sons.

[26] Heath, D., Jarrow, R. \& Morton, A. 1992 Bond Pricing and the term structure of interest rates: a new methodology for contingent claims valuation. Econometrica 60, 77-106.

[27] Internet: Digital Libery of Mathematical Functions, http://dlmf.nist.gov/13.2.

[28] JAMET, P. 1970 On the convergence of finite difference approximations to one-dimensional singular boundary-value problems. Numer. Math. 14, 355-378.

[29] JiAnG, L. 1965 Existence and differentiability of the solution of a two phase Stefan problem for quasi-linear parabolic equations. Chinese Acta Mathematica Sinica 7, 481-496. 
[30] JIANG, L., BIAN, B. \& YI, F. 2005 A parabolic variational inequality arising from the valuation of the fixed rate mortgages. European Journal of Applied Mathematics, 16, 361383.

[31] JAnSON, S. \& TYSK, J. 2006 Feynman-Kac formulas for Black-Scholes-type operators. Bull. London Math. Soc., 38, 269-282.

[32] KAU, J. B., KeEnAN, D. C. 1995 An overview of the option-theoretic pricing of mortgages. J. Housing Res., 6, 217-244.

[33] Kohn, J. \& Nirenberg, L. 1967 Degenerate elliptic-parabolic equations of second order. Comm. Pure Appl. Math., 20, 191-872.

[34] McKean, H. P. 1965 Appendix: A free boundary problem for the heat equation arising from a problem in mathematical economics. Industrial Management Review, 6, 32-39.

[35] OLEǏNIK, O. A. 1964 A problem of Fichera. Doklady Akademii Nauk SSSR 157,1129-1133.

[36] Overbeck, L. \& RYdÉn 1997 Estimation in the Cox-Ingersoll-Ross Model Econometric Theory, 430-461

[37] PESkiR, G. \& ShIRYAEV, A. 2006 Optimal stopping and free-boundary problems, Lectures in Mathematics ETH Zurich, Birkhauser Verlag, Basel.

[38] VASICEK, O. A. 1977 An equilibrium characterization of the term structure. Journal of Financial Economics 5, 177-188.

[39] Xie, D., Chen, X. \& ChAdAM, J. 2005 A parabolic variational inequality arising from the valuation of the fixed rate mortgages. European Journal Applied Mathematics 16, 361-383.

[40] YANG, H. 2004 American put options on zero-coupon bonds and a parabolic free boundary problem. Int. J. Numer. Analysis and Modeling, 1, 203-215.

[41] Yin, J. X. \& WANG, C. P. 2007 Diffusion equations with degeneracy on the boundary. International Conference of Chinese Mathematicians, III , 217-237. 